\title{
THE ENIGMA OF DARWIN DIAGRAM
}

\author{
Miloje M. Rakočević \\ Department of Chemistry, Faculty of Science, University of Nish, Serbia \\ (E-mail: milemirkov@open.telekom.rs; www.rakocevcode.rs) \\ What can be said at all \\ can be said clearly, \\ and what we cannot talk about \\ we must pass over in silence. \\ Ludwig Wittgenstein ("Tractatus")
}

\begin{abstract}
According to my best knowledge, for the first time here is presented a hypothesis, that the one and only "accompanying diagram" in Darwin's famous book On the Origin of Species contains, may be, a hidden code. Direct inspection reveals that the Diagram, viewed as built of four parts [(two upper and two lower / two left and two right); (two with more and two with less branches / two with multiple and two with single branches)], corresponds to the logical square of the genetic code. When, however, viewed as built of two parts (upper and lower), then it corresponds with Shcherbak's diagram (Shcherbak, 1993, 1994) of four-codon and non-four-codon amino acids (AAs); not only by the form but also by the number of elementary quantities. The number of nucleons in the upper part of Shcherbak's diagram (four-codon amino acids) is determined by the Pythagorean law $\left(3^{\wedge} 2+4^{\wedge} 2=5^{\wedge} 2=25\right)$, meaning that the total number of nucleons makes the product of the number 25 and "Prime quantum 037" (925); and the number of branches in the lower part of Darwin's diagram is determined by the law of Plato $\left(3^{\wedge} 3+4^{\wedge} 3+5^{\wedge} 3=6^{\wedge} 3\right.$ = 216), meaning that the total number of branches makes the product of the number 216 and "First quantum 01" (216). On the other hand, in the lower part of the Shcherbak's diagram there are 60 of "Prime quantum 037" (2220), while in the upper part of the Darwin's diagram there are 60 of "First quantum 01" (60). There are $216+60=276$ branches (in total), and this number is also the number taken from a specific and unique arithmetical system. Furthermore, it is shown that Darwin, starting from the basic structure of the Diagram, formed a sophisticated structure which strictly corresponds to the arithmetical and /or algebraic structures that also appear to be the key determinants of the genetic code (GC). Among other correspondences, there is also one in the number of entities/quantities, as follows. According to Shcherbak's account the nucleon number within the amino acid constituents of GC (in their side chains $)$ is as follows: $[1 \times(\mathrm{G} 1+\mathrm{A} 15+\mathrm{P} 41+\mathrm{V} 43+\mathrm{T} 45+\mathrm{C} 47+\mathrm{I} 57+\mathrm{N} 58+\mathrm{D} 59+\mathrm{K} 72+\mathrm{Q} 72+\mathrm{E} 73$ $+\mathrm{M} 75+\mathrm{H} 81+\mathrm{F} 91+\mathrm{Y} 107+\mathrm{W} 130)]+[2 \mathrm{x}(\mathrm{S} 31+\mathrm{L} 57+\mathrm{R} 100)]=1443$. If Shcherbak's account is done, with an iteration more, for the number of atoms, the result is as follows: $[2 \times(\mathrm{G} 1+\mathrm{A} 4+\mathrm{C} 5+\mathrm{D} 7+$ $\mathrm{N} 8+\mathrm{T} 8+\mathrm{P} 8+\mathrm{E} 10+\mathrm{V} 10+\mathrm{Q} 11+\mathrm{M} 11+\mathrm{H} 11+\mathrm{I} 13+\mathrm{F} 14+\mathrm{Y} 15+\mathrm{K} 15+\mathrm{W} 18)]+[3 \times(\mathrm{S} 5+\mathrm{L} 13+$ $\mathrm{R} 17)]=0443$. On the other hand, within Darwin's diagram there are the next "branch" entities/quantities: 276 branches, plus 46 nodes, plus 10 branchings, in total 332. The significant differences are as follows: 1443-332 = 1111and 443-332 = 111, both determined by the unity change law. From these results it follows that Darwin with his Diagram anticipated the relationships not only in terrestrial code but in the genetic code as well, anywhere in the universe, under conditions of the presence of water, ammonia and methane, phosphine and hydrogen sulfide. If so, then Darwinian selection moves one step backwards in prebiotic conditions, where it refers to the choice of the life itself.
\end{abstract}




\section{Introduction}

As it is generally known, Darwin's book On the Origin of Species contains only a single illustration, an evolutionary tree in the form of a diagram (Figure 1.1). During the 155 years since the appearance of the first edition in 1859, this Diagram has been analyzed only qualitatively (Figure 1.2), but not quantitatively, and we shall, in this paper, do that for the first time. ${ }^{1}$ In doing so, we begin with the working hypothesis (for this and all other researches of the Diagram in future) that the diagram contains a hidden code, with strictly determined quantities, expressed in the number of branches - primary (principal, main) and secondary (minor, small) ${ }^{2}$, and also in the number of nodes and branchings; such a code, which would per se have to be biological, otherwise it would not make sense in this book, and the Diagram would not be styled as "accompanying diagram" but as an "attached diagram", or an ordinary illustration. Hence, the deeper implication of the hypothesis is that, despite the variations (and modifications) of organisms are spontaneous and random, they do not have complete freedom, but are limited by the regularity and validity of strict arithmetical and/or algebraic systems. (Cf. Box 1.)

\section{Box 1. Citation from 1994 (I)}

Rakočević, 1994, p. 14: “Darwin's diagram-binary tree, represents the first systematic informational approach to the analysis of the relations between organisms. This is the only diagram in his book Origin of Species (Darwin, 1859) and it represents a model for interpretations of origin of varieties, species, genera and higher systematic categories. By its essence, this diagram represents a code-model and code-system and by its completness and complexity it is the first example of the code model and the code system in science. Relations of the elements within this code system correspond to the relations of the elements (organisms) in natural systems. Intention (and a message) of the author of this diagram is absolutely clear: if the natural systems are at the same time the coding systems, the only adequate and complete way of description and interpretation of such systems would be the creation of adequate code models with adequately corresponding relations between the elements of one and the other model, i.e. natural system."

The analysis that we conducted showed that the relationships between these quantities are such that they are brought into mutual relationships by specific proportionalities and balances through the minimal differences in number, usually expressed in decimal units $( \pm 00, \pm 01, \pm 10$,

\footnotetext{
${ }^{1}$ In fact, this is the third time. The first time, it was twenty-three (Rakočević, 1991), and the second time, it was 20 years ago (Rakočević, 1994). But both times it was only a pilot study, which was to serve as the initial "trigger" for a comprehensive analysis, the results of which are now presented. (Rakočević, 1991, p.4: „This diagram represents a specific coding system and the code program“.) (Rakočević, 1994, p.16: as here in Box 1 and Box 2.)

${ }^{2}$ Primary branches go from the previous level (line) and they always reach the next level (and they are designated by letters). Secondary branches, however, fail to reach the next level, they are not finalized; they do not become a taxonomic category (a variety, species, and so on.)
} 
$\pm 11, \pm 100, \pm 111$ and so on $)^{3}$, with the validity of the principle of minimal change, and the principle of continuity. ${ }^{4}$ Moreover, all of these quantities were related and corresponding to the quantities (and their relationships) in the genetic code; with the number of codons, molecules, atoms, nucleons etc.

The obvious reason why this is so, is (according to our working hypothesis) the fact that Darwin in his Diagram built relationships taken from the specific and unique arithmetical and/or algebraic systems, based on which, as we now know, the genetic code was also built.

\section{Methodology}

Bearing in mind that the genetic code is the basic biological code, and that it has already been proven that its distinctions and classifications (within itself), are derived on the basis of physicochemical properties of the molecule, followed by (accompanied by) strict arithmetical and/or algebraic regularities and balances (Shcherbak, 1993, 1994, 2008; Damjanović, 1998, 2005, 2006; Verkhovod, 1994; Dragovich, 2009, 2011; Mišić, 2011; Négadi, 2009, 2014; CastroChavez, 2010, 2011; Dlyasin, 2011; Jokić, 1996; Rakočević, 1997, 1998, 2004, 2011, 2013), it makes sense, in analysis of the distinction and classification in Darwin's diagram, to apply the same methodology (or almost the same) by which the said regularities in the genetic code were discovered. This means that the number of branches, nodes and branchings must be determined in even and odd positions; along cross diagonals, and zigzag lines; for different parts of the Diagram, which basically boils down to the application of Mendeleevian methodology, that can be found in his original manuscript works (Kedrov, 1977).

B.M. Kedrov, who most carefully studied the archives of Mendeleev, said that he was unable to find that Mendeleev wrote about which methodology he had used in his researches. In contrast to this, handwritten sketches, drawings and diagrams show that Mendeleev clearly revealed his methodology. In the above mentioned book, Kedrov enclosed 16 photocopies (between 128 and 129 pages $)^{5}$, showing the Mendeleevian methodology; which is the same methodology as we applied in the analysis of the genetic code structure as well as in the analysis of Darwin's diagram.

\footnotetext{
3 "Surprisingly, the genetic code really privileges a number system and, even more unusual, the system is the decimal one" (Shcherbak, 2008, p. 157).

${ }^{4}$ Here we address the Mendeleev's principles of one element or one period change; But we also bear in mind the validity of these two principles in the genetic code (Swanson, 1984, p. 187).

${ }^{5}$ All of these copies, plus two tables, can be found on our website ("The Mendeleev's archive"). Those particularly significant are: a copy (copy I, p. 128) which demonstrates "the chemical patience (solitaire)"; copy IV, which presents the chemical elements in the even/odd positions, with a drawing which indicates the number of odd and even valences, and the atomic mass differences are presented using the Pythagorean method of determining the differences in tetraktis (by Mendeleev in n-aktis); and copy VIII with the diagonal relations drawn in the Periodic system table.
} 


\section{Preliminaries}

Already at first glance, it becomes immediately obvious that Darwin's diagram (Figure 1.1), composed of four parts (two upper and two lower / two left and two right); (two with more and two with less branches / two with multiple and two with single branches), corresponds to the logical square of the genetic code, in a reverse reading ${ }^{6}$ (Figure 2), as well as with Shcherbak's diagram at the same time (Figure 3), also in the reverse reading. ${ }^{7}$ Two lower trees are branched, multiple, and two on the top are linear, non-branched, with linear segments. In the lower left part of the Diagram, the tree consists of two large branches, and the tree on the right consists of only one. In the upper, left part of the Diagram, there are more singlet branches (eight), and on the right there are less branches (six). ${ }^{8}$

The correspondence with Shcherbak's diagram is as follows: the "heads" of amino acid molecules have the same number of nucleons each, and their bodies are completely different. It is (by analogy) similar to the Darwin's diagram: the singlet branches are implemented in the same number at every level, and the multiple branches in different number, changing from level to level.

But it is so at first glance. However, the second (deeper) look reveals a surprising fact: the total number of nucleons in the amino acid molecules in the upper part of Shcherbak's diagram is determined by the Pythagorean law $\left(3^{\wedge} 2+4^{\wedge} 2=5^{\wedge} 2=25\right)$, meaning that it is 25 of "Prime quantum 037" (925), and the number of branches in the lower part of Darwin's diagram is determined by the law of Plato $\left(3^{\wedge} 3+4^{\wedge} 3+5^{\wedge} 3=6^{\wedge} 3=216\right)$, meaning that the amount is 216 of the "First quantum 01" (216). On the other hand, in the lower part of the Shcherbak's diagram there are 60 of "Prime quantum 037" (2220), while in the upper part of the Darwin's diagram there are 60 of "First quantum 01 " (60). ${ }^{9}$ [A total of nucleons is $925+2220=3145$, and a total of branches is $216+60=276$, which is again a number taken from a specific and unique arithmetical system, as the first case (Figure 4).]

[Remark 3.1. If we look at the first column in Shcherbak's original Table (Table 1 in Shcherbak, 1994): 037, 370, 703, it is clear that the first two steps can be realized by all two-digit numbers, while the third step (through module 9) is possible only for number 037; for example $(037,370, \mathbf{7 0 3})$ versus $(038,380, \mathbf{7 2 2})$.]

\footnotetext{
${ }^{6}$ Positioning "from smaller to larger" in the genetic code is from the left to the right, and in Darwin's diagram it is from the right to the left.

${ }^{7}$ In Shcherbak's diagram the smaller part is in the upper part of the Diagram and the large part is down in the lower part of the Diagram, while in Darwin's diagram it is the opposite. However, as the first inversion (with respect to the genetic code) is essentially natural, the latter is completely random.

${ }^{8}$ This "first glance" refers to descendants that follow from the species "A" and "I", whereas for the remaining species (B, C, D, E, F on the left and G, H, K, L on the right), the situation is somewhat different, and that will be explained in the text which follows.

${ }^{9}$ All branches (the sum $60+216=276$ ) which are the descendants of all 11 species designated with large Latin letters at the bottom of the Diagram are included into this counting.
} 
Darwin's diagram contains a zeroth level (undesignated) and 14 levels more, designated by Roman numerals. At the bottom of the Diagram, there are 11 English alphabet letters, A-L, ${ }^{10}$ ommiting the 10th letter (the letter "J"). ${ }^{11}$ Because of this exclusion, the original input order: J10, K-11, L-12, (M-13) becomes the output of order K-10, L-11, (M-12). ${ }^{12}$ In support to the assumption that here the term of coding is already present, there is the fact that the branches are omitted only at the 10th level. ${ }^{13}$ On the other hand, it is also a fact that the omission of capital letters begins with "M" (the 13th, central letter in the English alphabet), and alignment of small letters on the second branch of the left tree begins (and continues) exactly with "m". In addition, only the levels 11, 12 and 13 are not marked with small letters, while all the others are.

The omitting of the 10th letter makes another distinction: only the letters after the 10th letter are put into a new sequence, they are "variable". However, the letters from the $1^{\text {st }}$ to the $9^{\text {th }}$ remain unchanged, they are "stable". From that fact it follows that the main part of the Diagram is bounded by the first and by the last stable letter, "A" and "I". The species of organisms that are designated with these letters differ in other formal characteristics. Hence, we can speak about two sets of species: the first set of two, and the second set of "other nine species". In the first set of species, the branches (below the 10th level) are oblique (oblique angle), while in the second set the branches are orthogonal; within the first set there are nodes and branchings whereas within the other set there are not. By this, both types of branches (oblique and orthogonal) exist in both parts of the Diagram, in the left part, A-F, and in the right part, G-L.

The above reconciliation: 10th letter vs 10th level; "M" vs "m"; significant omission of capital letters at the start level versus reordering of the $11^{\text {th }}, 12^{\text {th }}$ and $13^{\text {th }}$ letters $(K, L, M)$, as opposed to the exclusion of small letters at the top of the Diagram at the positions $11^{\text {th }}, 12^{\text {th }}$ and $13^{\text {th }}$; all these relationships represent a kind of the specific realization of similarity principle and the principle of self-symilarity. ${ }^{14}$

\footnotetext{
${ }^{10}$ In Darwin's words: „The accompanying diagram will aid us in understanding this rather perplexing subject. Let A to $\mathrm{L}$ represent the species of a genus large in its own country. These species are supposed to replase each other at unequal degrees, as is so generally the case in nature, and as is representes in the diagram by the letters standing at unequal distances" (The Origin of species, 1876, Chapter IV, p. 90).

${ }^{11}$ One might think that this omission is done because the two adjacent letters "I" and "J" are similar to each other, so that Darwin wanted to avoid confusion. We, however, believe that this is such a code, which requires the omission of only the $10^{\text {th }}$ letter, no matter how it looks.

${ }^{12}$ As if Darwin wanted to tell us something about these numbers; perhaps to present their uniqueness: [(11/11, 22/22, 33/33, ... 99/99), (12/21, 24/42, 36/63), (13/31, 26/62, 39/93)] (cf. Table A.1 in Appendix A).

${ }^{13}$ This absence of branches should not be confused with the fact that at every level the branches (taxonomic entities) from the previous level are finalized, so thus, branches whose development started at the 9th level are finalized at the tenth level.

${ }^{14}$ Future researches should show whether this self-similarity is of fractal and/or non-fractal nature. A significant fact with regard to this, is Darwin's insisting on the fact that the structure of the Diagram can also refer to various taxonomic categories. (The Origin of species, Chapter IV, p. 91: "When a dotted line reaches one of the horizontal lines, and is there marked by a small numbered letter, a sufficient amount of variation is supposed to have been accumulated to form it into a fairly well-marked variety, such as would be thought worthy of record in a systematic work"; Chapter XI, p. 303: "We may suppose that the numbered letters in italics represent genera, and the dotted
} 


\section{Results and discussion}

\subsection{Primary and secondary branches of species " $\mathrm{A}$ " and "I"}

In our working hypothesis, there is a presumption that the symmetry relationships make the basis for coding, and for that reason we have analyzed the number and arrangement of branches, nodes and branchings on the 15 levels of the Diagram, at first, in symmetrical systems " 2 × 5" and " $3 \times 5$ ", and then in systems derived from them. Such symmetrical systems are presented first in Table 1.1, Table 1.2 and Table 2.1.

The number of primary (main) branches on the left tree (starting with letter "a") and the right tree (starting with the ending letter "z"), for the species "A" and "I" is given in Table 1.1.. The branches are counted starting from the zeroth level onwards, until the ninth, by counting the number of branches between every two levels. The same result is, however, obtained when we follow the finalization (realization) of taxonomic entities at every next level (Table 1.2). In the latter case, we start counting with the first instead of the zeroth level and we end counting with the tenth instead of the ninth level (by this counting we realize that the number of branches is equal to the number of letters per level).

From the aspect of this vision, all primary branches are "finalized" (and marked with the corresponding small letters at the lower part of the Diagram and the unmarked ones are in the upper part of the Diagram); they are further classified into two classes: 1. Finalized, fixed (Table 1.3), and 2. Finalized, not-fixed (Tables 1.4 and 1.5). These first branches reach a certain level and do not develop further; as examples, we show the first such branch on the left tree $(s 2)$, and the first such branch on the right $(t 3)$.

If we take any of the two tables (Table 1.1 and Table 1.2) and look at the upper half of the large (left) and lower half of the small (right) tree (and vice versa), then, in this cross-connection, the number of branches is equal (28 and 28). ${ }^{15}$ But apart from these symmetrical proportionalities to the total number of primary branches $(28: 28=1: 1)$, there is one more such proportionality valid for the parts of the system $(20: 20=1: 1)$ (the total number of primary branches on the small tree equals the number of branches on the upper half of the large tree); ${ }^{16}$ and there are also the following proportionalities: $(36: 24=3: 2),(32: 24=4: 3),(8: 16: 24: 32=$ 1:2:3:4) etc.

In Table 2.1 we look at all primary branches, up to the $14^{\text {th }}$ level. However, prior to the analysis, an important issue should be considered. In fact, according to the said first counting procedure, on the tenth level there are no branches; according to the other procedure, however,

lines diverging from them the species in each genus. ... The horizontal lines may represent successive geological formations, and all the forms beneath the uppermost line may be considered as extinct.") As if the same fractal motif extended along the overall evolutionary lines.

${ }^{15}$ Is it just a curiosity, that number 28 is the second perfect number?

${ }^{16}$ The same or similar proportionalities exist for the number of nodes, as well as for the number of branchings, which will be discussed further. 
we say that on the tenth level, three branches on the left, and two branches on the right tree (which arrived from the previous ninth level) are finalized. Then, the question is whether, in this second sense, there are also branches (descendants) at the eleventh level? The answer was given by Darwin himself, ${ }^{17}$ from which it follows that all four levels of the upper part of the Diagram contain finalized branches, which arrived from the previous 10th level: 8 on the left and 6 on the right. $^{18}$

The first thing we see in Table 2.1 is that the number of branches in the upper part of the Diagram is equal to the number of branches in the lower part of the Diagram $(56+56=4 \times 28=$ 112 ); then, that the result of cross-linking system components (along the two zig-zag lines), the pattern 52/60, as well as the total number of branches (112), was taken from a specific and unique arithmetical system (Fig. 5). In addition, this number of branches (112) is just a permutation of the number $121\left(11^{\wedge} 2\right),{ }^{19}$ which is actually the number of secondary branches on both trees, for the two species, "A" and "I" (Table 2.2) ${ }^{20}$; and this number is also taken from a specific and unique arithmetical system, which we have already presented in the Preliminaries (Figure 4).

Figure 4 shows several things at the same time. First, it presents a clear and unequivocal arithmetical system which from, as we have seen, Darwin took (reconciled) the results for the total number of branches in the Diagram (276) as well as for the number of secondary branches from zero up to the 9th level of the Diagram, the number 121, for the species "A" and "I" (Table 2.2). But at the same time we see that these results follow from the determination by the first perfect number, the number 6 , which also appears to be the determinant of the genetic code (Figure B.2). ${ }^{21}$

[Remark 4.1. Secondary branches do not have branchings, while the primary branches have. As examples, the two positions at the first level on the left tree: from $a 1$ there is not, while from $m 1$ there is a branching; details about speaking in Section 4.4, in tables $3.1-3.3$ (the nodes and branchings), in relation to tables 4-1 - 4-5, where there are the sums of the primary and secondary branches.]

\footnotetext{
${ }^{17}$ The Origin of species, 1876, Chapter IV, p. 91: "In the diagram the process is represented up to the ten-thousandth generation, and under a condensed and simplified form up to the fourteen-thousandth generation."

${ }^{18}$ The Origin of species, 1876, Chapter IV, p. 94: "Hence very few of the original species will have transmitted offspring to the fourteen-thousandth generation. We may suppose that only one (F), of the two species which were least closely related to the other nine original species, has transmitted descendants to this late stage of descent. The new species in our diagram descended from the original eleven species, will now be fifteen in number.".

${ }^{19}$ Notice that square of $11\left(11^{2}=121\right)$ is zeroth case in logical-arithmetical arrangement presented in Table A.1; also, the tenth part of the fourth friendly number, 1210 [more exactly, the second member of the second pair (1184 $\& 1210)$ of friendly numbers].

${ }^{20}$ In addition, it is "arranged so" that the diagonal result changes, for 10/01, respectively: The pattern 52/60, valid for all primary branches (Table 2.1) was changed into the pattern 62/59 (Table 2.2), valid for secondary branches (cf. Section 4.6, first paragraph).

${ }^{21}$ More details on the determination of GC by perfect and friendly numbers see in Rakočević, 1997b, p. 60.
} 


\subsection{The riddle of the genetic code}

Table 2.2 is very significant. It is amazing that the sequence of quantities: $11,22,33,44,55$, 66,77 is realized. ${ }^{22}$ It is hard to believe that it could be a coincidence, especially if we know that just by these numbers a specific and unique arithmetical system, which is one of the most important determinants of the genetic code, is bounded (Table C.1 in Appendix C) (Rakočević, 2011a, Table 4; 2011b, Table 4). The understanding of that determination is easier by illustrations given in Appendix $\mathrm{C}$, where it is shown that the said arithmetical system contains the specific algebraic system, which also appears to be a significant determinant of the genetic code: it determines codon/amino acids assignment in relation to a classification into four diversity types of amino acids (AAs).

In Figure C. 1 the classification into four diversity types is shown, in linear and circular form; and Figure C.2 shows the manner in which the circular arrangement becomes a Table of Mendeleevian type, where the molecules are arranged, mutatis mutandis, in accordance with the principles of minimum change and continuity. But what is surprising is the fact that the quantities $(26,42,57,77)$, representing the number of atoms in this Table (Figure C.2) are "taken" from the arithmetical system, given in Table C.1 (in relation to Table C.2 and C.3), in a manner as shown in Survey C.1. According to the algebraic equations given in Survey C.2, the 25 codons encode for less complex, and 36 for more complex AAs (Table C.4).

\subsection{Darwin's solution to the riddle of the genetic code}

The missing link in the strict determination of the genetic code by an arithmetical (Table C.1) and an algebraic system (Survey C.2 in relation to Survey C.1) is actually in the Survey C.2. In fact, we do not know which quadruplet sequence is preceded by or which one follows a sequence of squares $\left(6^{\wedge} 2,5^{\wedge} 2,4^{\wedge} 2,3^{\wedge} 2\right)$; moreover, we do not know which sequence is initial, and if there is a more general law that all the sequences are connected with? Fortunately, there is an answer, and it is contained in Darwin's diagram (Figure $6 \& 7$ in relation to Tables 5, 6.1 and $7.1)^{23}$

The general law is actually a rule, analogue to Hückel's rule $\mathrm{N}=(4 \mathrm{n}+2)(\mathrm{n}=0,1,2,3 \ldots)$, according to which, one can calculate the number $\mathrm{N}$ as the number of $\pi$ electrons in the most

\footnotetext{
${ }^{22}$ Table 4.5 presents the missing 88 (all branches on the second tree, for the "A" and "I" species, in 3 x 5 arrangement, 0-14 level), and again Table 7.5 (primary branches in all 11 species , 0-9 level); in Table 5 there is the number 99 , also missing in this sequence.

${ }^{23}$ In relation to Table 6.1 there are Tables 6.2, 6.3, 6.4 and 6.5, in relation to Table 7.1 there are Tables 7.2, 7.3, 7.4 and 7.5.
} 
stable aromatic molecules; and by analogy, the number of chemical elements in the periods of the periodic system of Mendeleyev (2s, 6p, 10d, 14f ...). ${ }^{24}$ (Cf. Box 2.)

\section{Box 2. Citation from 1994 (II)}

Rakočević, 1994, p. 14: "The main idea, which is in the basis of the diagram-binary tree, is the realization of the logic of the systematization and classification, separation of the parts within the whole, as well as the regularity of the hierarchy of the levels. The accordance of this logic with the model of classification of the number systems with the number basis $\mathrm{N}=2(2 \mathrm{n}+1)(\mathrm{n}=0,1,2,3)$ is directly obvious. ... So, we have for $\mathrm{n}=0, \mathrm{~N}=2$, what corresponds to the division of binary tree to the left tree and the right tree. It corresponds also to the Darwin's discussion of the relations during the evolution only along two lines at the beginning of which 'species (A)' and 'species (I)' occures ... In the case when $\mathrm{n}=1, \mathrm{~N}=6$, and this again corresponds to the division of the tree, to the left and right tree, but in this case this division is strictly indicated by only one line, the line of the letter (species) F which has a positional value of exactly 6 (this is the sixth letter in alphabet) ... The next possible relation in the system of classification and in the logic of the level hierarchy is the case when $\mathrm{n}=2$ and $\mathrm{N}=10$. This situation corresponds to a reduction of all branch outputs to three and two outputs [on the 10th level] on the left and right tree ... In the latter case, $n=3$ and $\mathrm{N}=14$, what corresponds to the end-outputs of the branches (on the 14th level) when "we get eight species ..., all descended from (A)'...; 'and (I) will have been replaced by six ... new species'. ${ }^{25}$

By this rule, as we now see, the connection between the quadruplets of squares is determined, in a series of natural numbers, through a system of two and two linear equations, ${ }^{26}$ which are connected by an "inserted" intermedial equation. In the case of the genetic code these three equations are found in the third "quadrant" of the system in Figure 7 (correspondingly with Survey C.1 and C.2, as well as Table C.4), with the intermedial equation as Darwin's equation $(27+09=36)$, which is found in Table 5 and Table 6.1 ; it determines the number of primary branches in the "9 other species" (out of species "A" and "I").

\footnotetext{
${ }^{24}$ A second manner in which we write this formula is $\mathrm{N}=2(2 \mathrm{n}+1)(\mathrm{n}=0,1,2,3)$. A "half" of this formula, in the form $\mathrm{N}=(2 \mathrm{n}+1)(\mathrm{n}=0,1,2,3)$ is just a formula for calculation of the odd numbers and the number of atom orbitals: $1 \mathrm{~s}, 3 \mathrm{p}, 5 \mathrm{~d}, 7 \mathrm{f} \ldots$

${ }^{25}$ In addition to what was written 20 years ago, now some refinements are given. It is obvious that Darwin in several different ways makes distinctions corresponding to the Hückel's rule. Two ways are explicit, one in a set of letters, and another in the set of the branches. First, we present solutions in the set of letters. So, the case for $\mathrm{n}=0$, and $\mathrm{N}=$ 2 refers to the second letter of the alphabet (B), which begins the second set of species. [In the first set there are (A, I), while in the second set there are (B, C, D, E, F, G, H, K, L).] The case for $\mathrm{n}=1$ and $\mathrm{N}=6$, refers to the 6th letter (F), which separates the left tree from the right tree in the Diagram. The case for $\mathrm{n}=2$ and $\mathrm{N}=10$ refers to the 10th letter $(\mathrm{J})$, which is excluded. The case for $\mathrm{n}=3$ and $\mathrm{N}=14$, refers to the 14th letter (n), which for the first and for the last time appears on the 14th level. [Letter $n$ as $13^{\text {th }}$, the middle letter reading backwards.] The solutions in the set of branches are these: on the $2^{\text {nd }}$ level, a first fixed branch appears $(s 2)$; after the $6^{\text {th }}$ level there is no branching; on the $10^{\text {th }}$ level there is the finalization of the branches from the lower part of the Diagram, and on the $14^{\text {th }}$ level there is the finalization of the branches from the upper part of the Diagram.

${ }^{26}$ Two linear equations whose unknown quantities are linked with a plus sign and two are associated with a minus sign.
} 
Hückel's rule (more precisely, an analogue of the rule) is a generalization concerning the "travel" of quadruplet squares generated from a series of natural numbers, starting with quadruplet $1-2-3-4$, that is with $1^{\wedge} 2-2^{\wedge} 2-3^{\wedge} 2-4^{\wedge} 2$. But knowing now for this Darwin's generalization that contains Hückel's rule, (and is related to the squares), as well as for Darwin's Platonian solution, given in the Preliminaries, and it concerns cubes, a new question is: Is a generalization over the $n$-th degree possible $(n=1,2,3,4,5 \ldots)$ ? In our opinion, the answer to this question should include the Mendel's quadruplet, valid for „Die entwicklung der Hybriden in ihren Nachkommen". ["Bezeichnet $\mathrm{n}$ die Anzahl der charackteristischen Unterschiede an den beiden Stammflanzen, so gibt $3^{\mathrm{n}}\left(3^{\wedge} \mathrm{n}\right)$ die Gliederzahl der Kombinationsreihe, $4^{\mathrm{n}}\left(4^{\wedge} n\right)$ die Anzahl der Individuen, welche in die reihe gehören, und $2^{\mathrm{n}}\left(2^{\wedge} \mathrm{n}\right)$ die Zahl der Verbindungen, welche konstant bleiben.". $]^{27}$

\subsection{Nodes and branchings}

Now we observe the Diagram (Figure 1.1) compared to Table 3.1. At the zeroth level we find a node on the left tree as well as on the right tree. At the first level, there are two nodes on the left and one node on the right etc., until the ninth level, after which there is no node involvement. Some nodes branch and some do not. By this, one must notice that there is a branching only when one of the nodes is followed by at least two branches, which are finalized at the next level (and they are marked by letters). Thus, the node at the zeroth level on the left tree is at the same time a branching, while on the right it is not (Tables 3.2 and 3.3). It is easily seen that after the sixth level there is no more branching. [On the sixth level there are the following branchings: $m 6$ branches into $m 7$ and $l 7$ on the left; $z 6$ branches into $z 7$ and $w 7$ on the right.] This fact requires that, in the analysis of the number of all branches, except the splitting into the $5+5$ levels as in Table 4.1 we must analyze the splitting into $7+3$ levels $^{28}$ as in Table 4.2 , and then into the $3+4$ +3 levels as in Table 4.3; and into $3+2+2+3$ levels as in Table 4.4.

The analysis shows that the number of nodes, as well as the number of branchings, along the two diagonal lines, is balanced through changes by \pm 0 or \pm 1 . Thus, the number of nodes is $23 \pm 1$ (Table 3.1), and the number of branchings is $5 \pm 1$ in Table 3.2 and $5 \pm 0$ in Table 3.3. The same balances were carried out in the odd/even positions.

The essential connection of nodes and branchings allows the possibility of their addition: 46 nodes +10 branchings equals 56 group tree-entities (Tables 3.1 and 3.2) in correspondence with 56 primary branches as individual tree-entities, both in the lower and in the upper part of the Diagram (Table 1.1 and 1.2 in relation to Table 1.5).] That essential connection is related to the fact that both primary and secondary branches spring from the same nodes (Table 3.1). But what

\footnotetext{
27 "According to Mendel, such system is determined by the four entities, $1^{\mathrm{n}}-2^{\mathrm{n}}-3^{\mathrm{n}}-4^{\mathrm{n}}(\mathrm{n}=1,2,3 \ldots)$ : Stammarten - Konstante Formen - Glieder - Individuen ... Note that Mendel only uses the term Stammarten, i.e. Stammpflanzen for the first entity but not the mathematical expression $1^{\mathrm{n}}$ which we use for the explanation of the Mendel's idea" (Rakočević, 1994, p. 176).

${ }^{28}$ However, by branching, not only levels are classified into $7+3$, but that was also done through the distribution of branchings on the left and the right tree; on the left tree the 7 of them, and on the right 3 branchings.
} 
is "unacceptable" concerning the addition is that some nodes (the ones in which there is a branching) are included in the sum twice. However, the same kind of "the unacceptable" we find in the sums of the nucleon number in the two classes of amino acids within Shcherbak's diagram (cf. legend to Figure 3).

\subsection{Binary-code-tree in Darwin's Diagram}

If we exclude (in the part of Darwin's diagram which is generated from the root "A") the nodes without branching, then we, mutatis mutandis, obtain the source Darwin's diagram (Figure 1.3). And if all secondary branches are excluded from this source Diagram, and only two primary branches are left at each node we get a "clean" binary tree, which one hundred percent corresponds to the binary tree of the genetic code (Figure 1 in Rakočević, 1998, p. 284).

[Remark 4.2. Darwin diagrams in Figures 1.3, 1.4 and 1.5 preceded to the book The Origin of Species; first two (Figures $1.3 \& 1.4$ ) as singlet ilustrations, while the third (Figure 1.5) as a set of illustrations - a unifying set of four diagrams. (For details see: Fleming, 2013.) But, what is important for us here is the fact that the Diagram in Figure 1.5 Darwin made by hand, and in it there is a small letter " $\mathrm{j}$ ", but as a large letter does not appear; also, there is a large letter "M" too, which does not exist in Figure 1.1. Altogether is in favor of our hypothesis that Darwin on his diagrams dealing with three sets of letters: 1 . A \& I; 2. B, C, D, E, F; G, H, K, L and 3. M, N, O, $\mathrm{P}, \ldots, \mathrm{Z}$.]

And, as on the binary tree of the genetic code where there is only one possible alternative in each step, in Darwin's evolutionary binary tree there is only one possible alternative, as well. One by one, along a binary tree, in a very long evolutionary path, from generation to generation, the totality of alternatives (changes and modifications implemented through the process of selection) dismisses the great antinomy of the diversity of organisms (Box 3), the basis of which is the antinomy of the genetic code (Box 4). In other words, variations and modifications, which Darwin's text presents, cannot be arbitrary, but are determined and bounded by a specific and unique arithmetical and/or algebraic structures /systems, the basis of which are the following principles: the principle of symmetry, the principle of the minimal change and the continuity principle.

\subsection{The balances of the number of branches for two species ("A" and "I")}

The number of primary branches for two species, "A" and "I", at all levels (I-XIV) is given by the pattern $52+60=112$ (Table 2.1$)^{29}$, which appears to be the middle case in a specific arithmetical system (Figure 5). On the other hand, the total number of secondary branches (from the zeroth to the ninth level) is such that it represents the change in 10/01 in relation to the number of primary branches, respectively: $52 / 60$ in Table 2.1 is changed to 62/59 in Table 2.2 $(52+10=62$ and $60-01=59)$. But what is rather surprising is that the unit balances continue further, going from one subsystem to the other within the system of the whole of Darwin's diagram.

\footnotetext{
${ }^{29} \mathrm{Cf}$. Section 4.1, paragraph 6, the first to the last.
} 


\section{Box 3. "Irreconcilable" antinomy of organism equality and diversity}

.A. Timiryazev, Istoricheskij metod v biologii, Akademiya nauk USSR, 1942, Moscow, p. 187-188: "If all organisms are related by the unity of origin (as it is proven by general observation derived from a comparison of fact classification, metamorphosis, comparative anatomy, embryology, paleontology), then the organic world [as opposed to the vast diversity] must be a merged, inseparable whole. That sharp contrast, that irreconcilable antinomy nobody managed to resolve neither before nor after Darwin. And he himself used to stop at it, until he found a solution that, logically, followed from the same principle - the principle of selection ... Natural selection provides a better chance of survival to those beings who possess some characteristics which ensure their survival under given conditions. Among such characteristics, there is some degree of difference in relation to the other closest beings and it saves them from the competition and provides, so to speak, some space for the newcomer. Thus, a differentiation, a certain degree of difference will be useful, it will mean the success of those forms which are the most different from their parents and from each other. Darwin called this the principle of characteristic divergence (divergence of characters) and he explained it by the following scheme (Figure 15 on p. 188)" (here: Figure 1.3).

\section{Box 4. "Irreconcilable" antinomy of the genetic code constituents equality and diversity}

The genetic code antinomy can be expressed in several ways, out of which we here present only two. The first way is Shcherbak's diagram itself (Figure 3): Within 15 identical "heads" of 15 nonfour-codon AAs there is the same number of nucleons, as in their 15 completely different bodies (1110). On the other hand, the number of nucleons within eight four-codon AAs - in different bodies, identical heads and whole molecules - is such as to comply with the law of Pythagoras (squares of numbers 3, 4 and 5, multiplied by the "Prime Quantum 037", respectively). Despite the fact that 19 out of 20 canonical AAs are derivatives of the same AA (glycine), they build a huge number of different proteins; and the four nucleotide bases, which are derivatives of the same molecule (pyrimidine), build a number of different and various DNA/RNA macromolecules, genes and genomes.

Thus, the total number of branches (primary + secondary branches, in the classification into $5+5$ levels), shown in Table 4.1, along the two diagonal lines is such that it constitutes a change of \pm 01 compared to the arithmetic mean, i.e. compared to the value of the central pair of numbers: the result $90 / 87$ in relation to $89 / 88$. In the next step (primary + secondary, in the classification into $7+3$ levels) as shown in Table 4.2 , a change by \pm 10 in the result $90 / 87 \rightarrow$ $80 / 97^{30}$ is realized. In the next step (primary + secondary branches, in the classification into $3+$ $4+3$ ) as shown in Table 4.3, the arithmetic mean, i.e. the central pair of numbers $(88 / 89)$ is realized.

Classifications and distinctions in Tables 4.1-4.4 do not affect the number of branches at even and odd positions, respectively, which is $82 / 95 ;{ }^{31}$ but in the fourth step (Table 4.4), in the result of the two zigzag lines, there is a change in \pm 01 exactly related to the result $(82 / 95 \rightarrow$

\footnotetext{
${ }^{30}$ As a result of splitting the arrangement $5+5$ into $7+3$, a specific self-similarity also appears through the patterns (46/44 versus 66/64) in Tables 4.1 and 4.2 , respectively.

${ }^{31}$ The change of \pm 02 is in relation to the diagonal result $80 / 97$ in Table 4.2.
} 
83/94). The fifth step is associated with a number of branches, from the upper part of the Diagram as well (arrangement $5+5+5$ ) (Table 4.5), and the result of the two zigzag lines represents a change of \pm 02 related to the arithmetic mean $(116 / 117 \rightarrow 114$ / 119).

\subsection{The "Prime Quantum 037"}

It is clear, from the results presented so far, that the key principle of classification is actually a (symmetric) distinction of the system, a splitting into two parts, in proportion 1:1 (5:5). Concerning the distinction 7:3, however, there must be some additional (hidden?) ${ }^{32}$ reason; maybe the appearance of the "Prime quantum 037" or a connection to Lucas's sequence (Figure D.1), or something else? But whatever it may be, the analysis of quantitative relations in the Diagram shows that precisely this distinction (Table 4.2), with the sub-distinction 3:4:3 (Table 4.3) is the most significant. Taken together, in unity, they show that the quantities are chosen in such a way that in the final result (along the diagonal lines) they represent the realization of $3^{\text {rd }}, 2^{\text {nd }}$ and $1^{\text {st }}$ of multiples of "Prime quantum 037". Moreover, they show (the sub-distinction in Table 4.3) that the "Prime Quantum 037" is a part of a broader arithmetical system (Table B.1 and Survey B.1) ${ }^{33}$ what we have also presented in several previous works, which from here we present just one (Rakočević, 2008, Tab. 3).

Interestingly, in an also hidden way, the "Prime Quantum 037" is also found in Mendeleev's calculations. ${ }^{34}$ At this point Mendeleev calculates the differences of atomic masses of elements, and in three cases makes two "mistakes". Instead of writing 30/27/67, what is actually the result, he writes 30/37/77 (Appendix B, Survey B.4).

\subsection{Primary and secondary branches for "other nine species"}

Table 5 provides an overview of the number of branches for the remaining nine species, B-F on the left part and G-H \& K-L on the right part. First, we see the number of primary branches at all levels (I-XIV): $27+09=36$ (Table 6.1), ${ }^{35}$ as a result through which Darwin solves "the riddle of the genetic code" (Section 4.3). [Review of counting through levels for primary branches is given in Tables 6.1 and 6.2.] On the right of the result, in Table 5, the result of the total number

\footnotetext{
${ }^{32}$ L.N. Tolstoy (by Pierre Bezukhov in "War and Peace"): "Today my benefactor revealed me a part of the secret. He spoke about a large outer space square and he told me that the third and the seventh number are the basis of everything".

${ }^{33} \mathrm{Cf}$. the result 66 in the upper part and 037 in the lower part in Table 4.3 with the same pattern (66/037), also 66 in the upper part and 037 in the lower part, in Survey B.1.

${ }^{34}$ Kedrov, 1977, p. 128 , photocopy X. Having found the result where Mendeleev allegedly made a mistake in two out of three cases (!?), Kedrov concluded that even the greatest can make a mistake. In our opinion, Mendeleev did not make a mistake, he actually made his (hidden) code, which strictly corresponds to the Darwin's. (cf. Survey B.4).

${ }^{35}$ The results shown in Tables 6.1-6.5 refer to the "other nine species", while the results for the "all 11 species" are shown in Tables 7.1-7.5; in all of these tables, the letters on the two final branches, instead of the previous designation with small letters "a" and "z" now have the designations â and $\hat{z}$, with circumflex accent.
} 
of secondary branches is given $(3+4=7),{ }^{36}$ from the zeroth to the sixth level, because there are none of them on other levels, as shown by the specific counting in the Diagram (Table 6.3). ${ }^{37}$ Therefore, the total number of branches (primary + secondary) for "other nine species," from the zeroth to the 14th level is $36+07=43$ (Table 6.4), and from the zeroth to the 9th level is $32+07=39$ (Table 6.5). ${ }^{38}$

In Table 6.1 we see that the number of primary branches for "other nine species", at $0-14$ levels, is balanced in the odd/even positions, as well as along the two zigzag lines $(18+18)$. It is clear that there is balance at levels $0-9$ in odd/even positions $(16+16)$, and that there is no balance for four units of the two diagonal lines (Table 6.2). For secondary branches the balance in the same spatial situations is realized with \pm 1 difference (3/4) (Table 6.3); for the sum of primary and secondary branches (at levels 0 -14) the balance is also realized with \pm 1 difference (21/22) (Table 6.4), and this balance is disrupted for three units at 0-9 level (Table 6.5).

\subsection{Primary and secondary branches for all 11 species}

Table 7.1 shows that in Darwin's diagram, we find a total of 276 branches; a number that, in union with the number 121 (which represents the total number of secondary branches of "first two species", "A" and "I"), represents the first case of a specific and unique arithmetical system (as we have shown in the Preliminaries and in Figure 4). The total number of branches splits into two sets, 60 branches in the upper part of the Diagram (with singlet branches) and 216 branches (Plato's number!) in the lower part of the Diagram, with multiple branches (Table 7.2). ${ }^{39}$ Table 7-2 also shows that the number of branches of the first and of the second five levels, represents a change of \pm 10 in relation to the arithmetic mean of the total number of branches in the lower part of the Diagram $[(216: 2=108) ;(108+10=118) ;(108-10=98)]$. The same model is valid for the whole Diagram, for the total number of primary (Tables 7.3) and secondary branches (Table $7.4)$, but in relation to the total number of branches, number $276[(276: 2=138) ;(138+10=$ $148) ;(138-10=128)]$.

\footnotetext{
${ }^{36} \mathrm{Cf}$. this result $\mathbf{0 7}$ for the total number of secondary branches (at 0-6 level, i.e. at1-7. level), in „other nine species“, with 07 primary finalized and fixed branches in „first two species“ ("A" and "I", in Table 1.4) at 0-7 level, i.e. at 1-8 level.

${ }^{37}$ As we see, Darwin's splitting into $7+3$ levels is given not only in the logic of branching (the nodes for the "first two" species "A" and "I"), but also in the logic of the secondary branches layout (in levels) for the "other nine species." Moreover, this logic is given for the third time as well, in the right part of the Diagram, for the "other four species" (G-H and K-L) not any branch, neither primary nor secondary, is present at the levels after the sixth. [Notice that "nine other species" are splitting into five on the left, and four on the right.]

${ }^{38} \mathrm{Cf} .39$ all branches in "other nine species" (Tab.6.5) with all 49 primary, finalized non-fixed branches in the "first two species" ("A" and "I") (Table 1.4).

${ }^{39}$ In the Preliminaries we have presented that here, there is also the relation between the "final" result in the genetic code (60 of "Prime Quantum 037" and 5^2 x 037) and the "final" result in Darwin's diagram (60 of "First Quantum $01 "$ and $1 \times 6^{\wedge} 3$ ). And the relation between the numbers 2220 and 925 in the GC is obvious (in fact it is both times determined by Pythagorean Law) while in Darwin's diagram the relation between 60 and 216 is almost unnoticeable. In the absence of a more obvious insight, we now present a possible regularity: $60=5 \times(6+6)$ and $216=6 \times(6 \times 6)$.
} 
Table 7.5 presents the results of the total number of branches from the zeroth to the ninth level, as in Table 7.4, of the total number of secondary branches. (A Table in analogy with Table 7.3 for the secondary branches is not possible, because there are no secondary branches in the upper part of the Diagram.) In addition to the other balances, Table 7.5 shows an obvious determination through the sequence of a series of natural numbers: 42, 43, 44, 45, 46.

\subsection{Improbable and unexpected result}

In Section 4.3 we have shown that Darwin's equation naturally "fits" the two linear equations which determine the connection between codons and amino acids. And there is nothing surprising in that. Darwin understood (and there is no doubt about that) the existence of a specific and unique system, and with that system he adjusted his (hidden) code stored in the Diagram. However, there is another, perhaps more direct link with the genetic code, for which there is almost no explanation. This connection is revealed by comparing Darwin's result, presented in Table 4.3 to the result which represents the number of atoms in the amino acid molecules, as it is shown in the standard GC Table, if Shcherbak's calculation method is applied.

Shcherbak's calculation procedure is as follows: the number of nucleons in one-meaning AAs is taken into account once, and in two-meaning AAs (, S, R) twice. ${ }^{40}$ Thus, for example, for the number of nucleons in side chains of AAs he got the following result: $[1 \times(\mathrm{G} 1+\mathrm{A} 15+$ $\mathrm{P} 41+\mathrm{V} 43+\mathrm{T} 45+\mathrm{C} 47+\mathrm{I} 57+\mathrm{N} 58+\mathrm{D} 59+\mathrm{K} 72+\mathrm{Q} 72+\mathrm{E} 73+\mathrm{M} 75+\mathrm{H} 81+\mathrm{F} 91+\mathrm{Y} 107+$ $\mathrm{W} 130)]+[2 \times(\mathrm{L} 57+\mathrm{S} 31+\mathrm{R} 100)]=\underline{\mathbf{1}} 443$. If, however, Shcherbak's calculation procedure, is performed with an iteration more, for the number of atoms, the result is as follows: $2 \mathrm{x}(\mathrm{G} 1+$ $\mathrm{A} 4+\mathrm{C} 5+\mathrm{D} 7+\mathrm{N} 8+\mathrm{T} 8+\mathrm{P} 8+\mathrm{E} 10+\mathrm{V} 10+\mathrm{Q} 11+\mathrm{M} 11+\mathrm{H} 11+\mathrm{I} 13+\mathrm{F} 14+\mathrm{Y} 15+\mathrm{K} 15+$ $\mathrm{W} 18)]+[3 \mathrm{x}(\mathrm{S} 5+\mathrm{L} 13+\mathrm{R} 17)]=\underline{\mathbf{0}} 443$. On the other hand, the number of all "branch" entities/quantities in Darwin's diagram is: 276 branches (Table 4.5 in relation to Table 5) plus 46 nodes (Table 3.1) +10 branchings (Table 3.2) equals 332. From this result, the significant differences in relation to GC are: $1443-332=1111$ and 443-332 = 111, in both cases determined by a strict balance, expressed through the law of unity change (four and three unit positions, respectively). But that is not all. If the above iteration is derived in a Mendelevian system of AAs (Table E.1) we get the result of two parts which are related to each other also through the unit change law: $277-166=111$. What is, however, surprising is the fact that this result written in the

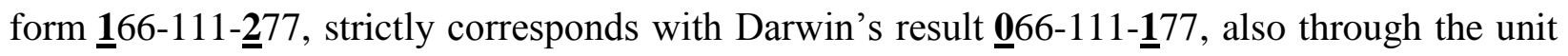
change law (cf. Table 4.2 with Table E.1). From all these results it follows that Darwin's diagram contains a prediction of relationships not only in terrestrial but the genetic code anywhere in the universe, under conditions of the presence of water, ammonia and methane, phosphine and hydrogen sulfide. If so, then Darwinian selection moves one step backwards in prebiotic conditions, where it refers to the choice of the life itself.

${ }^{40}$ One-meaning AAs are decoded by the codons from one codon family, but two-meaning AAs are decoded by codons from two codon families (L,S, R). 


\subsection{More than improbable result}

This raises the question: whether, perhaps, it is possible to find an arithmetical system that will show all Darwin's quantities, which he used to determine the relations in the Diagram, gathered in one place? Yes, this is the system shown in the Survey B.4. Even more than that, it is a system that demonstrates that Darwin's hidden code is in the unity with the Mendeleev's hidden code (Section 4.7), as well as with the genetic code (Survey B.5 in relation to Survey B.6 and B.7), and without that unity none of these three codes [one natural (genetic code) and two created (Mendeleev code and Darwin code)] can be understood.

\section{Concluding remarks}

1. Presenting in this paper a possible Darwin's hidden code, and the arguments in favor of the working hypothesis, given in the Introduction (for this and all other researches of the Diagram in future) of the actual existence of such a code, we hope that we are now also closer to the answer to Shcherbak's crucial question about the nature of arithmetical regularities in the genetic code. $^{41}$ The essence of Darwin's coding is that the principle of selection must also refer to the pre-biological conditions, when it comes to selection of life itself. In some way, unknown to us, Darwin grasped and understood that biological organization must be in correspondence with the organization of unique arithmetical and/or algebraic systems; precisely as we now know that it is so in the genetic code, as presented in this, and in the previous works of several authors. Hence, the whole Darwin's book On the Origin of species is actually a qualitatively expressed biological code and the diagram represents a quantitative evidence of the same code.

2. The working hypothesis, however, can only be considered as proven, provided that one should first understand (and that is our intention, so throughout the paper, we have provided arguments to support it) that Darwin consciously and deliberately encoded everything; in other words, it is proven that the relations presented in Darwin's diagram were not randomly presented. In addition to the aforesaid, it is enough to look at Figures 4 and 5 where two special arithmetical systems are presented, both in relation to the "arithmetical-logical square 11-12-13-14", presented in Table A.1. From the aspect of the probability theory the question is not the probability with which we can accidentally "extract" the numbers one by one, but three numbers at once [in Figure 4, the numbers are: 12-23-276, 23-34-782, etc., where the first case is Darwin's case (Table 7.1) ${ }^{42}$; in Figure 5 there are: 26-36-62, 52-60-112, etc., where the second case is

\footnotetext{
${ }^{41}$ In one of his first works in which he presented that the physico-chemical classification of the constituents of the genetic code is followed by arithmetical patterns and the balance of the number of particles (nucleons), V. Shcherbak concluded that "The physical nature of such a phenomenon is so far not clear" (Shcherbak 1993, last sentence).

42 The number 276 as the total number of branches within Darwin's diagram. Anyway, here within the set of "possible cases" there are all two-digit, three-digit and four-digit numbers, provided that the zeroth case $(1,12,12)$ is excluded; because, if it was involved, then single-digit numbers would be included as well, and the combinations would be - the combinations with repetition, so the probability would be even less.
} 
Darwin's case ${ }^{43}$ (Table 2.1) $]^{44}$. This, then, means that there is the question of the selection probability of not only these two arithmetical systems, but of all other arithmetical / algebraic systems presented here, correspondent with Darwin's quantities that appear as important determinants in the Diagram.

3. However, independently of the future, we present the probabilities for the two systems in Figures 4 and 5. The probability of a "favorable" event being realized, within the system in Figure 4 (for example, to "derive" the triple $12-23-276$ ) ${ }^{45}$, the probability is $1: 6 \times 10^{\wedge} 12$; and to derive all triples listed in Figure 4 (seven triples), the probability is $1: 10^{\wedge} 79$. As for the system in Figure 5, regarding the fact that the system reaches the end of the three-digit and not four-digit numbers, and that only four cases are presented, the probability is slightly higher $1: 10^{\wedge} 33$. But since these two systems are independent, with the independent events, the probability to draw both systems (in the given lengths) is $1: 10^{\wedge} 112$. It is clear that both systems in their totality, tend to reach the infinity, whereas the probability tends to reach zero, that is to say, to the impossible event.

Everything would be the same if we would like to determine the appereance probability for the elements of the system, presented in the Survey B.7 (which is in a conection with the system in Survey B.6). However, in favor of the intention and the disqualification of randomness, there is a fact of conditional probability occurrence: with the appearance of the triple 177-277-377, its analogue triple 066-166-266 automatically appears; then, with the triple $288-388-488$ there is its analogue 177-277-377 etc. In addition to this, there is one fact more: the first case is additionally significant, because it contains the Darwin's solution (177-066) in the first position, and the genetic code solution (277-166) in the second position (Table E.1).

4. Based on the findings, presented in this paper, it makes sense to set up a hypothesis (prediction!) according to which a future research will show that life, in all its levels (presented here in the unity and coherence of physical-chemical laws and arithmetical-algebraic regularities) is manifested in proportionalities and harmonious balance. ${ }^{46}$ In addition to that, we

43 The result $52+60=112$ as the number of primary branches within species "A" and "I" (Table 2.1). Anyway, within the set of "possible cases" there are all two-digit, three-digit and four-digit numbers, provided that the zeroth case $(0,12,12)$ is excluded; because, if it was involved, single-digit numbers would be included as well, and the combinations would be - the combinations with repetition, so the probability would be even less.

${ }^{44}$ Notice that arithmetical system in Figure 5 is a derivative of the system in Figure 4, of its first row.

${ }^{45}$ Having realized that this triple is an element of another system, as well (Table C.2), which is in a strict connection with the system in Table C.1, and which is a direct determinant of the genetic code (the determinant of assignment of codons to amino acids, classified into four types of diversity), the calculation of probability practically loses its point; it becomes immediately obvious that intentions, and not coincidences are present here. At the same time, it becomes clear how and why the structure of Darwin's diagram corresponds with the structure of the genetic code, although, in the time when he lived, Darwin could not know anything about the genetic code. Simply, Darwin understood relations in arithmetical systems, presented in Tables C.1 and C.2, based on which, as we now know, the genetic code was also built.

46 “.... and in the systems of distant celestial spheres ... changes, similar to those which happen in front of us during the chemical reactions of particles, have been happening up to now. A future Newton will discover the laws of these changes, as well. And, although the chemical changes are unique, they are, however, just variations on the general theme of harmony which regins in the nature" (Mendeleev, 1958, p. 554). 
expect that the results presented here will help in resolving some dilemmas - Darwinism or Intelligent design, ${ }^{47}$ as well as the dilemma: if cultural evolution is subject to Darwinian selectionism or is it a "communal exchange" (Gabora, 2013; Kaufman, 2014). ${ }^{48}$

5. It is so with hypothesis for the future, but if I am to express my opinion, here and now, just based on these results, then, here it is: Concerning the intelligent design, I have nothing to add to what I said in the previous work (here: footnote 47). As for culture, I believe that professors L. Gabora and S. Kaufman (footnote 48) are wrong. As a Darwinian selection has to move one step backwards in prebiotic conditions, it has to move one step forward, as well, where it refers to human consciousness and its "products," such as human society.

All kinds of "communal exchanges" are primarily found in the input, and when it comes to the final output (which language and which culture survive and which languages and cultures disappear), they must necessarily be the result of Darwinian selection, as the most general law valid for all manifestations of life, starting with the problem of its origin in the immaterial, through all the manifestations of actual life, until the problem of appearance and manifestation of consciousness and meaningfulness, including the evolution of human society itself.

\section{Acknowledgement}

I am very grateful to Branko Dragovich, Vladimir Ajdačić and Tidjani Negadi for helpful, stimulating and benevolent discussion about the genetic code, during the last decade, and for a critical reading of this paper and very useful suggestions related to it.

\footnotetext{
${ }^{47}$ Rakočević, 2013, p. 10: "With insight into the results ... one is forced to propose a hypothesis (for further researches) that here, there really is a kind of intelligent design; not the original intelligent design, dealing with the question - intelligent design or evolution (Pullen, 2005), which is rightly criticized by F.S. Collins (2006). Here, there could be such an intelligent design, which we could call "Spontaneous Intelligent Design" (SPID) that is consistent with that design which was presented by F. Castro-Chavez (2010), and is also in accordance with the Darwinism. [F. Castro-Chavez (2010, p. 718): "We can conclude that the genetic code is an intelligent design that maximizes variation while minimizing harmful mutations."] Actually, it can be expected that the hypothetical SPID, contained in the results ..., is in accordance with an identical (or similar?) SPID, presented in the only diagram, in Darwin's book "Origin of Species" (Darwin, 1996), as we have shown through an analysis of that diagram in one of our books (Rakočević, 1994; www.rakocevcode.rs). [In the case of the statement that spontaneity and intelligent design are mutually opposite, one must ask the question: isn't it true that human intelligence is the result of a spontaneous evolutionary process?]"

${ }^{48}$ Kaufman, 2014, p. 1: "As Gabora points out, ideas and artifacts get put to new uses and combined with one another in new ways for new functionalities, and this is what underlies technological, cultural and political evolution. None of this is captured or even approachable by way of a Darwinian theory of culture. Gabora does two things in this paper. First, she levels a reasoned and devastating attack on the adequacy of a Darwinian theory of cultural evolution, showing that cultural evolution violates virtually all prerequisites to be encompassed by Darwin's standard theory. Second, she advances the central concept that it is whole world views that evolve."
} 


\section{F I G UR E S}
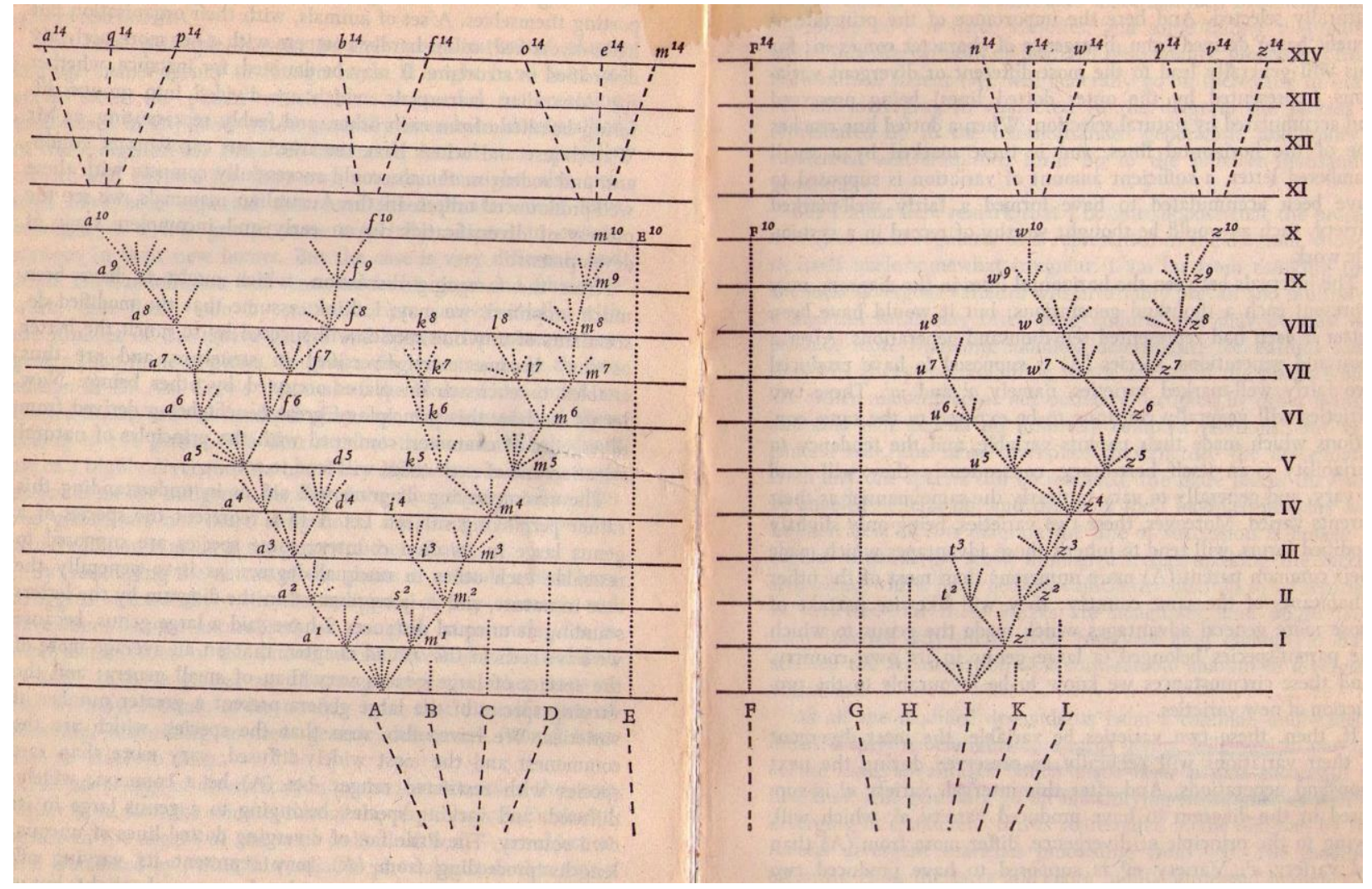

Figure 1.1. The "accompanying diagram" in Darwin's book "On the Origin of Species" (London, 1859) 


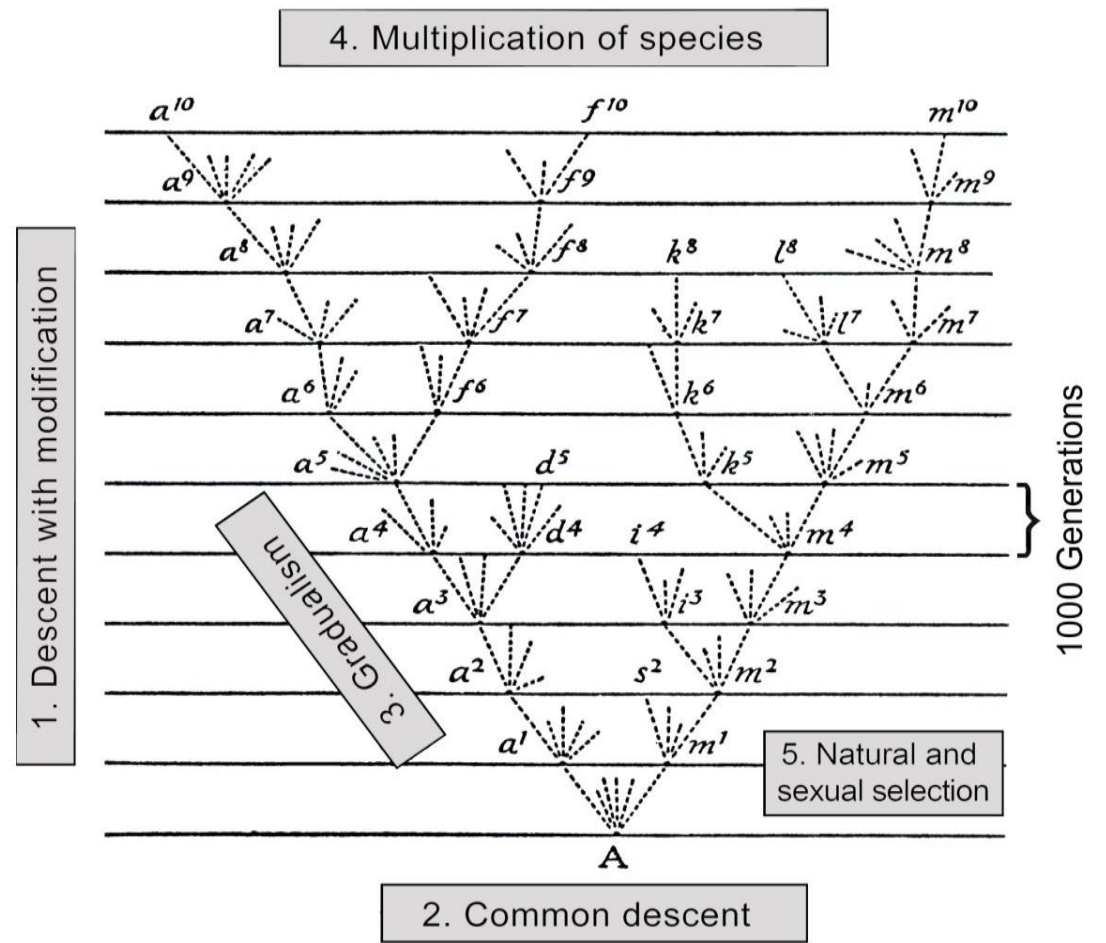

Figure 1.2. The qualitative analysis of Darwin's diagram (www.biologydirect/darwin) 


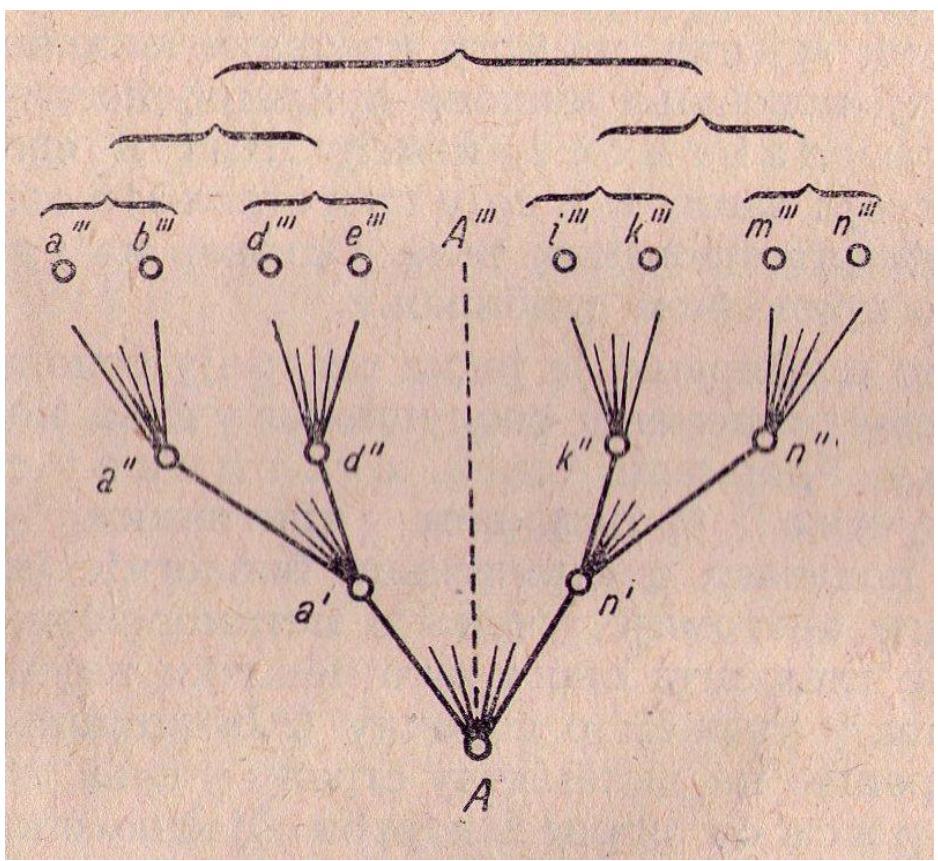

Figure 1.3. The Darwin's binary tree in his initial, preliminary draft „The foundations of origin of species“, 1842 (after: Kliment A. Timiryazev, Istoricheskij metod v biologii, Akademiya nauk SSSR, 1942, Moskva, Figure 15 on p. 188). 


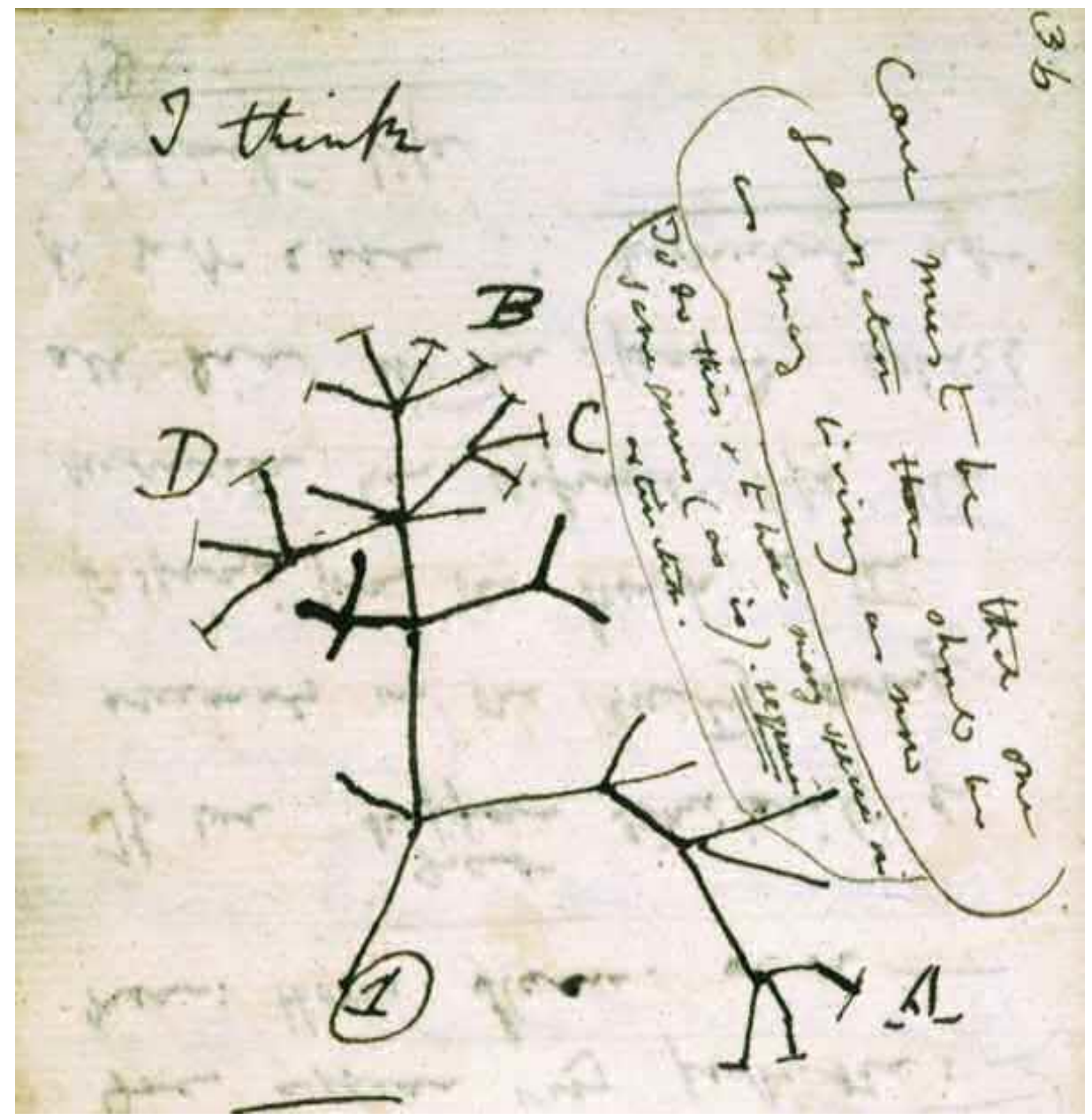

Figure 1.4. In mid-July 1837 Darwin started his "B" notebook on Transmutation of Species, and on page 36 he wrote "I think" above his first evolutionary tree. 


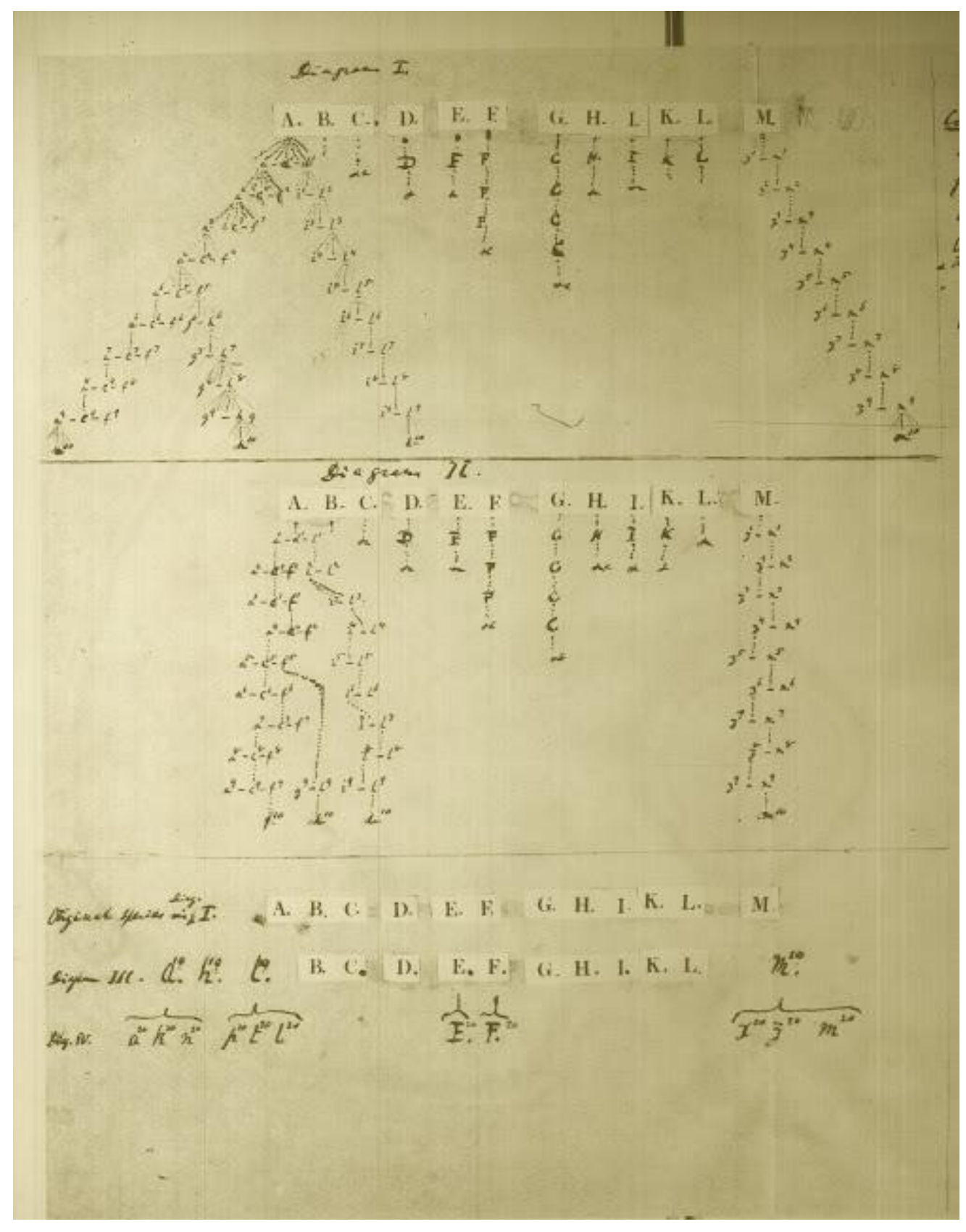

Figure 1.5. In mid-July 1837 Darwin started his "B" notebook on Transmutation of Species, and on page 36 he wrote "I think" above his first evolutionary tree. 


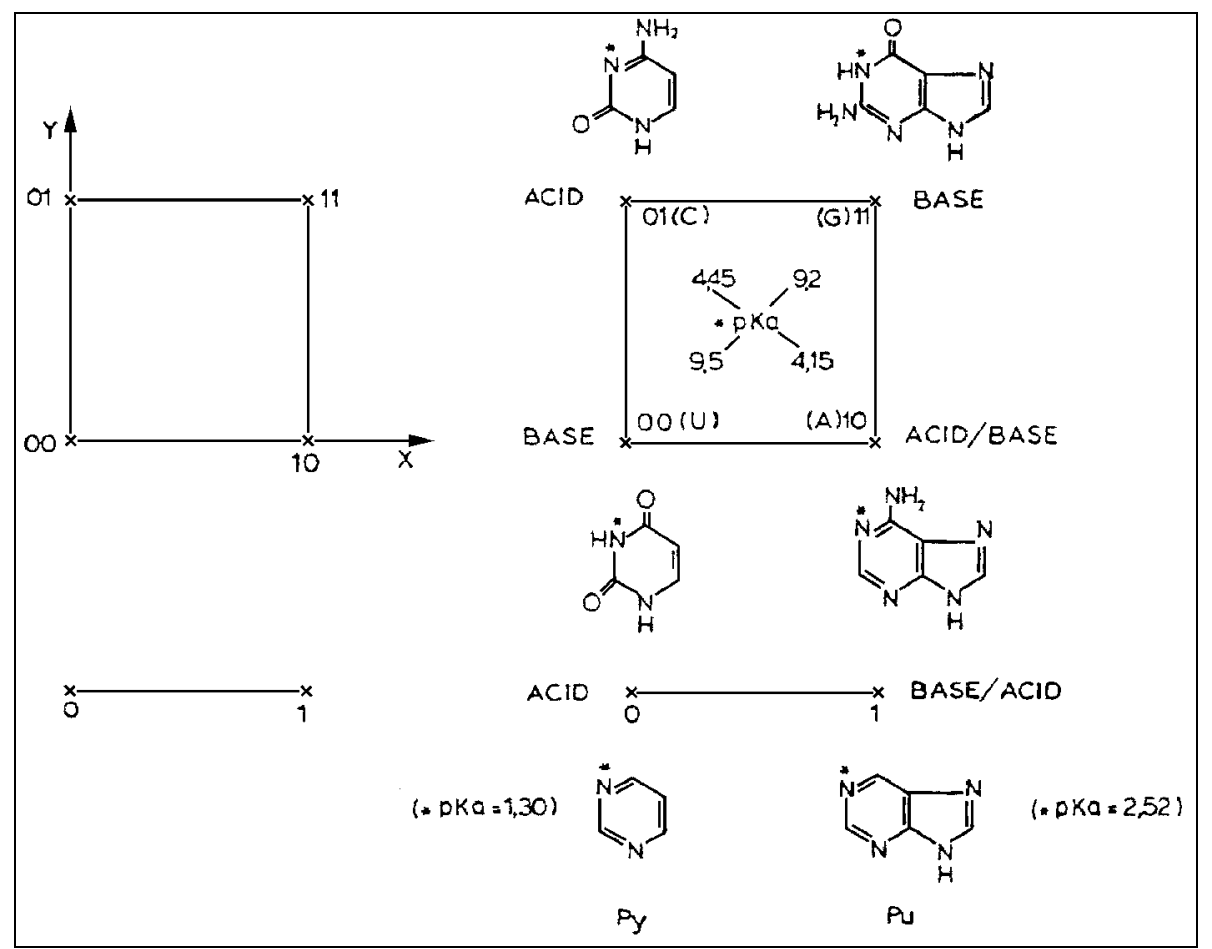

Figure 2. The logic square of the Genetic code: two single versus two double molecules; two with two and two with three hydrogen bonds (after: Rakočević, 1994, p. 8). 


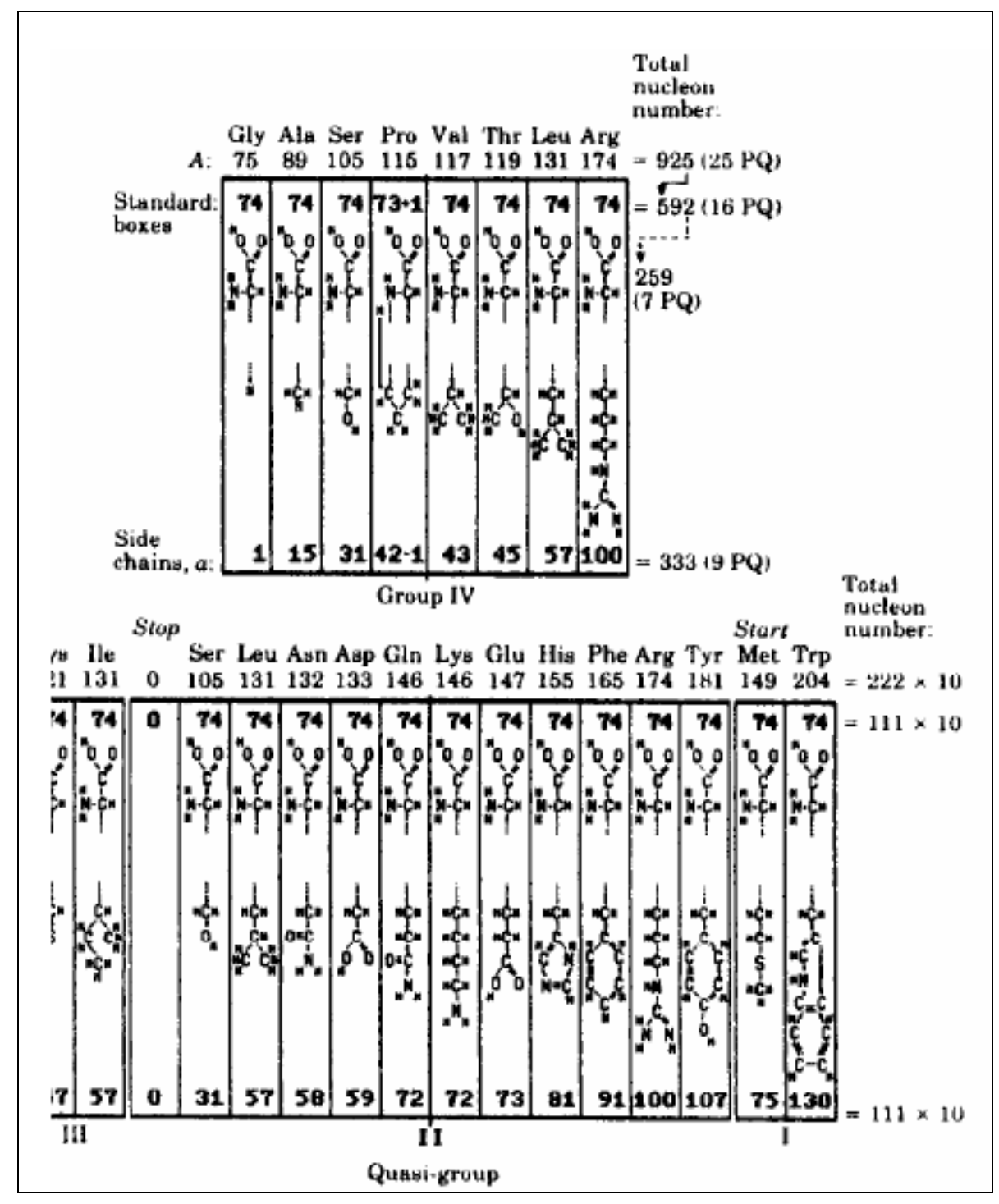

Figure 3. The Shcherbak's diagram of classification into four-codon and non-four-codon amino acids. The one-meaning AAs are included in the sum once while two-meaning AAs (L, S, R) are included twice (Shcherbak, 1994, Fig. 1 ). 


\begin{tabular}{|c|c|c|c|c|c|c|c|c|}
\hline$\left(0^{\text {th }}\right)$ & 01 & $x$ & 12 & $=$ & 012 & & & \\
\hline$\frac{(1)}{\left(1^{s t}\right)}$ & 12 & $X$ & 23 & $=$ & 276 & 264 & $242^{(6 \times 044)}$ & \\
\hline (2) & & & & & & 506 & & \\
\hline$\left(2^{\text {nd }}\right)$ & 23 & $\mathrm{X}$ & 34 & $=$ & 782 & & 242 & 121 \\
\hline (3) & & & & & & 748 & & \\
\hline$\left(3^{\text {rd }}\right)$ & 34 & $x$ & 45 & $=$ & 1530 & & 242 & \\
\hline (4) & & & & & & 990 & $(6 \times 165)$ & \\
\hline$\left(4^{\text {th }}\right)$ & 45 & $x$ & 56 & $=$ & 2520 & & 242 & \\
\hline (5) & & & & & & 1232 & & \\
\hline$\left(5^{\text {th }}\right)$ & 56 & $x$ & 67 & $=$ & 3752 & & 242 & 121 \\
\hline (6) & & & & & & 1474 & & \\
\hline$\left(6^{\text {th }}\right)$ & 67 & $x$ & 78 & $=$ & 5226 & & 242 & \\
\hline (ㅁ) & & & & & & 1716 & $(6 \times 286)$ & \\
\hline$\left(7^{\text {th }}\right)$ & 78 & $x$ & 89 & $=$ & 6942 & & 242 & \\
\hline
\end{tabular}

Figure 4. The multiples of $[(1+11 n)(12+11 n)](n=0,1,2, \ldots)$. The pattern ,276“ appers to be Darwin's determinant as the total number of branches in the Diagram (Table 7.1); as well as the pattern „121“ which also appears to be Darwin's determinant as the total number of secondary branches for two species (A and I) in the Diagram (Table 2.2). 


\begin{tabular}{|c|c|c|c|c|c|c|c|}
\hline 0 & $X$ & 13 & $=$ & 00 & \multirow[t]{2}{*}{12} & \multirow[t]{2}{*}{$\underline{0} 12$} & \multirow[b]{2}{*}{50} \\
\hline 1 & $X$ & 12 & $=$ & 12 & & & \\
\hline 2 & $x$ & 13 & $=$ & 26 & \multirow{2}{*}{10} & \multirow{2}{*}{062} & \\
\hline 3 & $X$ & 12 & $=$ & 36 & & & 50 \\
\hline 4 & $x$ & 13 & $=$ & 52 & \multirow{2}{*}{08} & \multirow{2}{*}{$\underline{112}$} & \\
\hline 5 & $X$ & 12 & $=$ & 60 & & & \\
\hline 6 & $X$ & 13 & $=$ & 78 & \multirow{2}{*}{06} & \multirow{2}{*}{162} & \\
\hline 7 & $x$ & 12 & $=$ & 84 & & & \\
\hline 8 & & 12 & & 101 & \multirow[b]{2}{*}{04} & \multirow[b]{2}{*}{$\underline{212}$} & \\
\hline 9 & $\hat{x}$ & 12 & $=$ & 108 & & & \\
\hline $0=$ & & (49) & 12 & $=170)$ & (170 & & \\
\hline
\end{tabular}

Figure 5. The multiples of numbers 13 and $12 ; 13$ by even, and 12 by odd numbers from natural numbers sequence. The Darwin's pattern $(52+60=112)$ is presented in the dark tones area in Table 2.1. 


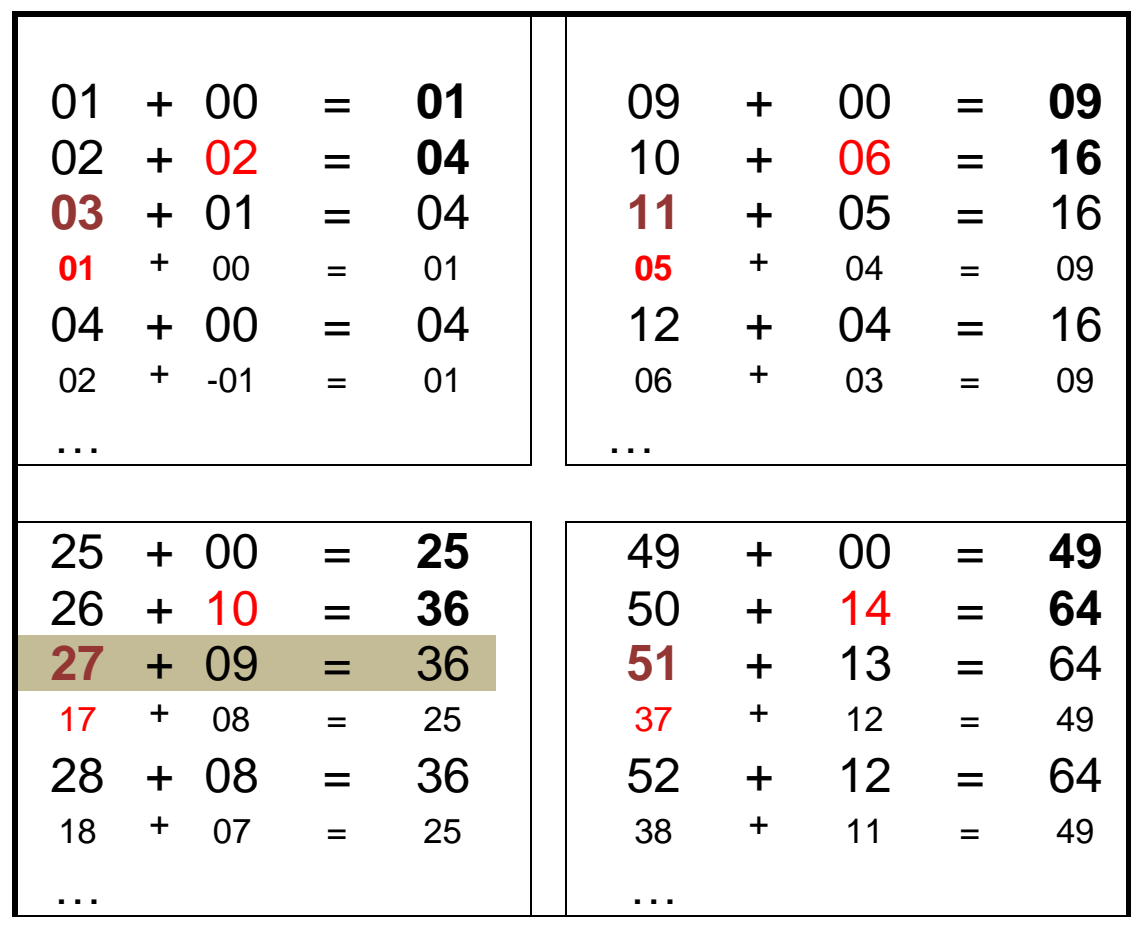

Figure 6. The generation of the squares of natural numbers through two linear equations. Darwin's equation is in the third quadrant, in the area of dark tones (Tables 5 and 6.1) surrounded by two linear equations valid in the genetic code (Table C.2), presented in Survey C.2. 


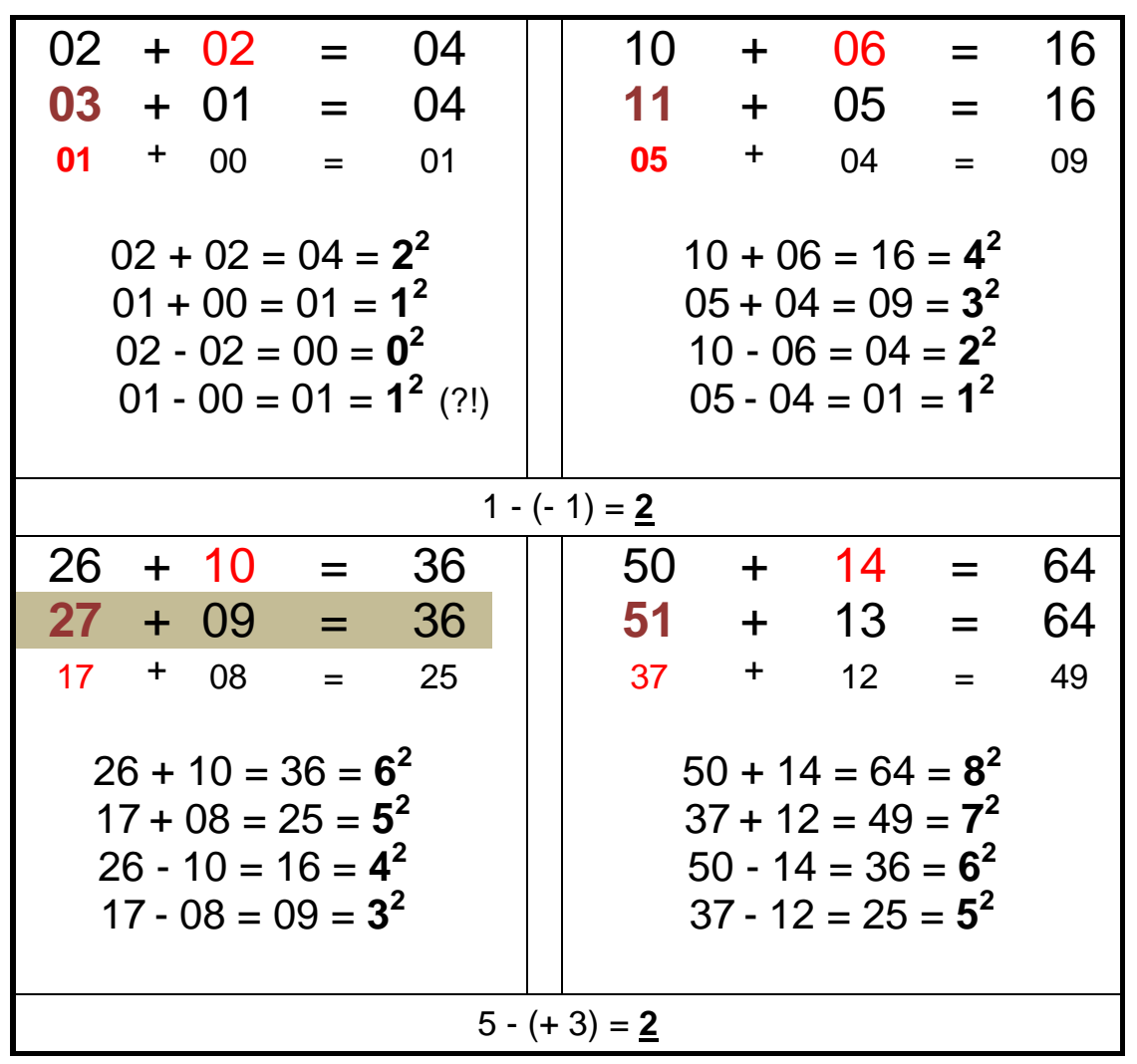

Figure 7. This Figure follows from the previous one, Figure 6. Three linear equations within each of the four quadrants in relation to the quadruplets of natural numbers' squares. In the third quadrant: two equations are valid in the genetic code (Table C.2) and one (in the middle position, dark tone) is given as Darwin's equation (Tables 5 and 6.1). [Notice a paradox (Darwin's paradox), valid for number 1 in the first quadrant: the negative value of number 1 cannot be - negative?!] 


\begin{tabular}{|c|c|c|c|c|c|c|}
\hline $1^{\wedge} 2$ & + & $2^{\wedge} 2$ & + & $3^{\wedge} 2$ & $=$ & 14 \\
\hline $1^{\wedge} 1$ & + & $2^{\wedge} 1$ & + & $3^{\wedge} 1$ & $=$ & 06 \\
\hline $1^{\wedge} 3$ & + & $2^{\wedge} 3$ & + & $3^{\wedge} 3$ & $=$ & $6^{\wedge} 2$ \\
\hline 1 & & 8 & & 27 & & \\
\hline & 9 & & + & 27 & $=$ & 36 \\
\hline$G$ & $\mathrm{H}$ & $\mathrm{K}$ & L & & & \\
\hline $6: 1$ & $2: 1$ & $0: 1$ & $1: 1$ & $3^{\wedge} 2+$ & $3^{\wedge} 3$ & $=6^{\wedge} 2$ \\
\hline 8 & & 1 & & & & \\
\hline & & 9 & & $9+$ & 27 & $=36$ \\
\hline $\mathrm{x}^{\wedge} \mathrm{n}$ & + & $\mathrm{y}^{\wedge} \mathrm{n}$ & $=$ & $\begin{array}{l}\mathrm{z}^{\wedge} \mathrm{n}-1 \\
\end{array}$ & \multirow{4}{*}{\multicolumn{2}{|c|}{$\begin{array}{l}\text { Valid only for } \\
\mathrm{n}=3\end{array}$}} \\
\hline$x^{\wedge} 3$ & + & $y^{\wedge} 3$ & $=$ & $\mathrm{z}^{\wedge} 2$ & & \\
\hline $1 \wedge 3$ & + & $2^{\wedge} 3$ & $=$ & $3^{\wedge} 2$ & & \\
\hline 1 & & 8 & $=$ & 9 & & \\
\hline
\end{tabular}

Figure 8. The relationships between the first three natural numbers. On the top area: the first row shows that the sum of the the first three numbers' squares equals 14 - a half of the second perfect number; the second row shows the sum of the first three numbers as the first perfect number, the number 6; the third row shows that the sum of the cubes of the first three numbers equals the square of the first perfect number; in the fourth row we see the values which follow from the third row; the fifth row shows the Darwin's equation (Tables 5 and 6.1). In the central area, on the left there is the number of primary (bold) and secondary branches, valid for the species G, H, K, L and on the right there is a part of Darwin's diagram. [Notice that there are two manners to understand Darwin's approach for a splitting into $8+1=9$ branches: in relation to ,species-I“" position (left G \& H and right K \& L); and in relation to the zeroth position (there is no primary branches in K position).] In the middle area, on the right: the second variant of the generation of Darwin's equation; the second, in relation to the 5th row in the top area. Down: the intermedial step in generation of Darwin's equation is shown $(1+8=9)$. 


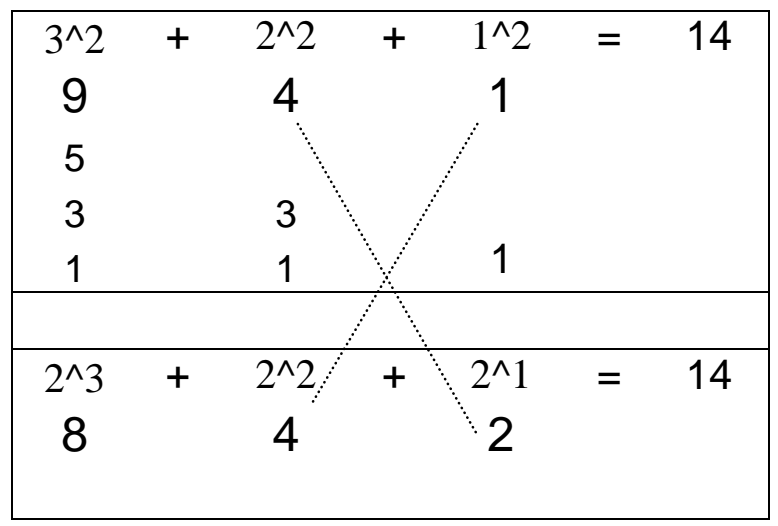

Figure 9. The relationships within the periodic system of chemical elements (PSE) in correpondence with the equation which we have taken from the first row in Figure 8; also in correpondence with the reverse form of this equation. The arrangement is as follows: 5 elements of $s$-type or $p$-type, 3 elements of $d$-type and 1 element of $f$-type. This pattern is realized (in Periodic Table) 8 times; The following pattern has 3 elements of $d$-type and 1 element of $f$-type, and it is realized 2 times; Finally, we have the form of 1 element of $f$-type, which is repeated four times. [Cf. Table 18, p. 180 in Rakočević, 1997b; by this one must notice that in PSE, in Table 18, there are 1+14 groups ("1" as zeroth group), analogously to 1+14 elements in Mendeleev's Table: 1 is the lanthanum and 14 are the lanthanides (the last, lutetium, was not known for the life of Mendeleyev, but he is still indicated it, as it is presented in Table 16, in Kedrov, 1977, p. 188); also, analogously to $1+14$ levels in Darwin's diagram.] 
T A B L E S

\begin{tabular}{|c|c|c|c|c|}
\hline $\begin{array}{ll}\underline{a}_{9} & 03\end{array}$ & $02 \underline{Z}_{9}$ & $\underline{a}_{9} \quad 03$ & & $02 \underline{\mathbf{Z}} \mathbf{9}$ \\
\hline$a_{8} \quad 03$ & $02 Z_{8}$ & $a_{8} 03$ & & $02 Z_{8}$ \\
\hline$\underline{a}_{7} \quad 05$ & $03 \underline{Z}_{7}$ & $\underline{\mathbf{a}}_{7} 05$ & & $03 \underline{Z}_{7}$ \\
\hline$a_{6} \quad 05$ & $03 Z_{6}$ & $a_{6} 05$ & & $03 Z_{6}$ \\
\hline $\begin{array}{ll}\underline{a}_{5} & 04\end{array}$ & $02 \underline{Z}_{5}$ & $\underline{a}_{5} 04$ & & $02 \underline{Z}_{5}$ \\
\hline$a_{4} \quad 04$ & $02 Z_{4}$ & $a_{4} 04$ & & $02 Z_{4}$ \\
\hline$\underline{a}_{3} \quad 04$ & $01 \underline{\mathbf{Z}}_{3}$ & $\underline{\mathbf{a}}_{\mathbf{3}} \quad 04$ & & $01 \underline{Z}_{3}$ \\
\hline$a_{2} \quad 03$ & $02 \bar{Z}_{2}$ & $\bar{a}_{2} 03$ & $16(2$ & $02 \bar{Z}_{2}$ \\
\hline $\begin{array}{ll}\underline{a}_{1} & 03\end{array}$ & $02 \underline{Z}_{1}$ & $\underline{\mathbf{a}}_{1} 03$ & & $02 \underline{Z}_{1}$ \\
\hline$a_{0} \quad 02$ & $01 \bar{Z}_{0}$ & $a_{0} 02$ & & $01 Z_{0}$ \\
\hline Odd 19 & (29)10 & 27 & & \\
\hline Even 17 & 10 & 29 & $\begin{array}{c}28 / 28 \\
(00)\end{array}$ & \\
\hline 36 & 20 & 56 & 56 & \\
\hline
\end{tabular}

Table 1.1. All primary branches at $0-9$ levels (for two species: A and I) in the splitting $(5+5)$. The counting starts from every initial level at which the branching occurs $(\underline{\mathbf{0}}-1, \underline{\mathbf{1}}-\mathbf{2}, \underline{\mathbf{2}}-\mathbf{3}, \ldots, \underline{\mathbf{9}}-10)$, and the $9^{\text {th }}$ level is the last. 


\begin{tabular}{|c|c|c|c|c|}
\hline$a_{10} \quad 03$ & $02 Z_{10}$ & $a_{10} 03$ & & $02 Z_{10}$ \\
\hline $\begin{array}{ll}\underline{a}_{9} & 03\end{array}$ & $02 \quad \underline{Z}_{9}$ & $\begin{array}{|ll|}\underline{\mathbf{a}}_{9} & 03\end{array}$ & & $02 \quad \underline{Z}_{9}$ \\
\hline$a_{8} \quad 05$ & $03 Z_{8}$ & $a_{8} 05$ & & $03 Z_{8}$ \\
\hline $\begin{array}{ll}\underline{\mathbf{a}}_{7} & 05\end{array}$ & $03 \quad \underline{\mathbf{Z}}_{7}$ & $\underline{\mathbf{a}}_{7} \quad 05$ & & $03 \underline{\mathbf{Z}}_{7}$ \\
\hline $\begin{array}{ll}a_{6} & 04\end{array}$ & $02 \quad Z_{6}$ & $a_{6} \quad 04$ & & $02 \quad Z_{6}$ \\
\hline $\begin{array}{ll}\underline{\mathbf{a}}_{5} & 04\end{array}$ & $02 \quad \underline{Z}_{5}$ & $\begin{array}{ll}\underline{\mathbf{a}}_{5} & 04\end{array}$ & & $02 \underline{Z}_{5}$ \\
\hline $\begin{array}{ll}a_{4} & 04\end{array}$ & $01 Z_{4}$ & $a_{4} \quad 04$ & & $01 Z_{4}$ \\
\hline$\underline{\mathbf{a}}_{3} 03$ & $02 \underline{\mathbf{Z}}_{3}$ & $\begin{array}{ll}\underline{\mathbf{a}}_{3} & 03\end{array}$ & & $02 \underline{Z}_{3}$ \\
\hline $\begin{array}{ll}a_{2} & 03\end{array}$ & $\begin{array}{ll}02 & Z_{2}\end{array}$ & $a_{2} \quad 03$ & & $02 z_{2}$ \\
\hline $\begin{array}{ll}\underline{\mathbf{a}}_{\mathbf{1}} & 02\end{array}$ & $01 \underline{\mathbf{z}}_{1}$ & $\begin{array}{ll}\underline{\mathbf{a}}_{1} & 02\end{array}$ & & $01 \underline{\mathbf{Z}}_{1}$ \\
\hline $\begin{array}{cc}\text { Even } & 19 \\
\text { Odd } & 17\end{array}$ & $\mid \begin{array}{ll}(29) & 10 \\
(27) & 10\end{array}$ & $\begin{array}{l}(27) \\
(29)\end{array}$ & $28 / 28$ & \\
\hline 36 & 20 & 56 & 56 & \\
\hline
\end{tabular}

Table. 1.2. All primary branches at $1-10$ levels (for two species: A and I) in the splitting (5+5). The counting starts from each subsequent level at which the branch is finalized $(1,2,3, \ldots, 10)$, and the $10^{\text {th }}$ level is the last. 


\begin{tabular}{|c|c|c|c|c|}
\hline$a_{10} 00$ & $00 Z_{6}$ & $a_{10} 00$ & & $00 Z_{10}$ \\
\hline$\underline{\mathbf{a}}_{9} \quad 00$ & $00 \underline{Z}_{9}$ & $\underline{\mathbf{a}}_{\mathbf{9}} 00$ & & $00 \underline{Z}_{9}$ \\
\hline$a_{8} \quad 02$ & $01 Z_{8}$ & $a_{8} 02$ & & $01 \quad Z_{8}$ \\
\hline$\underline{a}_{7} \quad 00$ & $00 \underline{Z}_{7}$ & $\underline{\mathbf{a}}_{7} 00$ & & $00 \mathbf{Z}_{7}$ \\
\hline$a_{6} \quad 00$ & $00 Z_{6}$ & $a_{6} 00$ & & $00 Z_{6}$ \\
\hline$\underline{a}_{5} \quad 01$ & $00 \underline{Z}_{5}$ & $\underline{\mathbf{a}}_{5} \quad 01$ & & $00 \underline{Z}_{5}$ \\
\hline$a_{4} 01$ & $00 Z_{4}$ & $a_{4} 01$ & & $00 Z_{4}$ \\
\hline$\underline{\mathbf{a}}_{3} \quad 00$ & $01 \underline{\mathbf{Z}}_{3}$ & $\underline{\mathbf{a}}_{3} 00$ & & $01 \underline{Z}_{3}$ \\
\hline$a_{2} 01$ & $00 Z_{2}$ & $a_{2} 01$ & & $00 Z_{2}$ \\
\hline $\begin{array}{ll}\underline{\mathbf{a}}_{1} & 00\end{array}$ & $00 \underline{Z}_{1}$ & $\underline{\underline{a}}_{1} 00$ & & $00 \underline{Z}_{1}$ \\
\hline $\begin{array}{l}\text { Even } 04 \\
\text { Odd } 01\end{array}$ & $\begin{array}{l}01 \\
\text { (05) } 01 \\
\text { (02) }\end{array}$ & $\begin{array}{l}(02) \\
(05)\end{array}$ & $03 / 04$ & \\
\hline 05 & 02 & 07 & 07 & \\
\hline
\end{tabular}

Tab. 1.3. All primary, finalized, fixed branches at 1-10 levels (for two species: A and I) in the splitting $(5+5)$. The counting is as in Table 1.2. (Notice the results in the form of the sequence: $1,2,3,4,5$.) 


\begin{tabular}{|c|c|c|c|c|c|}
\hline$a_{10}$ & 03 & $02 Z_{10}$ & $a_{10} \quad 03$ & & $02 Z_{10}$ \\
\hline$\underline{a}_{9}$ & 03 & $02 \quad \underline{Z}_{9}$ & $\underline{a}_{9} \quad 03$ & \multirow{4}{*}{$18(29) 11$} & $02 \quad \underline{Z}_{9}$ \\
\hline$a_{8}$ & 03 & $02 \quad Z_{8}$ & $a_{8} \quad 03$ & & $02 Z_{8}$ \\
\hline$\underline{a}_{7}$ & 05 & $03 \quad \underline{Z}_{7}$ & $\underline{\mathbf{a}}_{7} \quad 05$ & & $03 \quad \underline{Z}_{7}$ \\
\hline$a_{6}$ & 04 & $02 \quad Z_{6}$ & $a_{6} \quad 04$ & & $02 Z_{6}$ \\
\hline$\underline{a}_{5}$ & 03 & $02 \quad \underline{Z}_{5}$ & $\underline{\mathbf{a}}_{\mathbf{5}} \quad 03$ & & $02 \underline{Z}_{5}$ \\
\hline $\bar{a}_{4}$ & 03 & $01 \bar{Z}_{4}$ & $\bar{a}_{4} \quad 03$ & & $01 \bar{Z}_{4}$ \\
\hline$\underline{a}_{3}$ & 03 & $01 \quad \underline{Z}_{3}$ & $\underline{a}_{3} \quad 03$ & & $01 \boldsymbol{Z}_{3}$ \\
\hline $\bar{a}_{2}$ & 02 & $02 \bar{Z}_{2}$ & $\bar{a}_{2} \quad 02$ & $13(20) 07$ & $02 \bar{Z}_{2}$ \\
\hline$\underline{\mathbf{a}}_{1}$ & 02 & $01 \underline{Z}_{1}$ & $\underline{\mathbf{a}_{1}} \quad 02$ & & $01 \underline{\mathbf{Z}}_{1}$ \\
\hline $\begin{array}{l}\text { Even } \\
\text { Odd }\end{array}$ & $\begin{array}{l}15 \\
16\end{array}$ & $\begin{array}{l}24) 09 \\
(25) 09\end{array}$ & $\begin{array}{l}25 \\
24\end{array}$ & $25 / 24$ & \\
\hline & 31 & 18 & 49 & 49 & \\
\hline \multicolumn{6}{|c|}{$(31-20=11)(29-18=11)$} \\
\hline \multicolumn{6}{|c|}{ Fixed $7\left(7^{1}\right)+49\left(7^{2}\right)$ non-fixed $=56$ primary } \\
\hline
\end{tabular}

Tab. 1.4. All primary, finalized, non-fixed branches at 1-10 levels (for two species: A and I) in the splitting (5+5). The counting is as in Table 1.2. 


\begin{tabular}{|c|c|c|c|c|}
\hline$a_{14} 08$ & $06 Z_{14}$ & $a_{14} 08$ & & $06 Z_{14}$ \\
\hline$\underline{\mathbf{a}}_{13} \quad 08$ & $06 \underline{Z}_{13}$ & $\mathbf{a}_{\mathbf{1 3}} 08$ & & $06 \underline{Z}_{13}$ \\
\hline $\bar{a}_{12} \quad 08$ & $06 \bar{Z}_{12}$ & $a_{1208}$ & $32(56) 24$ & $06 Z_{12}$ \\
\hline$\underline{\mathbf{a}}_{\mathbf{1 1}} 08$ & $06 \underline{Z}_{11}$ & $\underline{a}_{11} 08$ & & $06 \underline{Z}_{11}$ \\
\hline$a_{10} 00$ & $00 Z_{10}$ & $a_{10} 00$ & & $00 Z_{10}$ \\
\hline$a_{10} 03$ & $02 Z_{10}$ & $a_{10} 03$ & & $02 Z_{10}$ \\
\hline $\begin{array}{ll}\underline{\mathbf{a}}_{\mathbf{g}} & 03\end{array}$ & $02 \underline{Z}_{9}$ & $\underline{a}_{9} 03$ & & $02 \underline{Z_{9}}$ \\
\hline$a_{8} \quad 03$ & $02 Z_{8}$ & $a_{8} 03$ & $18(29$ & $02 Z_{8}$ \\
\hline$\underline{\mathbf{a}}_{7} \quad 05$ & $03 \underline{Z}_{7}$ & $\underline{\mathbf{a}}_{7} 05$ & & $03 \underline{Z}_{7}$ \\
\hline$a_{6} \quad 04$ & $02 Z_{6}$ & $a_{6} 04$ & & $02 Z_{6}$ \\
\hline $\begin{array}{ll}\underline{a}_{5} & 03\end{array}$ & $02 \underline{Z}_{5}$ & $\underline{a}_{\mathbf{5}} 03$ & & $02 \mathbf{Z}_{5}$ \\
\hline$a_{4} \quad 03$ & $01 Z_{4}$ & $a_{4} 03$ & & $01 Z_{4}$ \\
\hline$\underline{\mathbf{a}}_{\mathbf{3}} 03$ & $01 \underline{Z}_{3}$ & $\underline{\mathbf{a}}_{\mathbf{3}} 03$ & $13(20) 07$ & $01 \underline{Z}_{3}$ \\
\hline$a_{2} \quad 02$ & $02 Z_{2}$ & $a_{2} 02$ & & $02 Z_{2}$ \\
\hline $\begin{array}{ll}\underline{\mathbf{a}}_{\mathbf{1}} & 02\end{array}$ & $01 \underline{Z}_{1}$ & $\underline{\mathbf{a}}_{\mathbf{1}} 02$ & & $01 \underline{Z}_{1}$ \\
\hline $\begin{array}{l}\text { Even } 31 \\
\text { Odd } 32\end{array}$ & $\mid \begin{array}{ll}(52) & 21 \\
(53) & 21\end{array}$ & $\begin{array}{l}53 \\
52\end{array}$ & $49 / 56$ & \\
\hline 63 & 42 & \multicolumn{3}{|c|}{$105(216-111)$} \\
\hline \multicolumn{5}{|c|}{$(105=56+49)[233-105=128(121+7)]$} \\
\hline
\end{tabular}

Tab. 1.5. All primary, finalized, non-fixed branches on 1-14 levels (for two species: A and I) in the splitting (3x5). The counting is as in Table 1.2. Notice the self-similarity expressed through quantities on two zigzag lines: 49 as non-fixed branches (Table 1.4), 56 as total number of primary branches in the lower as well as in the upper part of the Diagram (Table 2.1). The result 105 follows from this distinction: all 112 primary branches (Table 2.1) minus 7 fixed branches (Table 1.3). The balance and self-similarity: 105 as all primary, finalized, non-fixed branches $=216$ as all the branches in the lower part of the Diagram (0-9 levels, for all 11 species) minus 111 "undefined" units. [Self-similarity is present here because 111-105 = 6 and 177 (in Table 4.1) minus 111 equals 66 as in Table 4.2 (Notice the determinants 6 and 66 in Table B.1).] 


\begin{tabular}{|c|c|c|c|c|}
\hline$a_{14} 08$ & $06 Z_{14}$ & $a_{14} 08$ & \multirow{5}{*}{$32(56) 24$} & $06 Z_{14}$ \\
\hline$\underline{\mathbf{a}}_{\mathbf{1 3}} \quad 08$ & $06 \underline{Z}_{13}$ & $\underline{\mathbf{a}}_{\mathbf{1 3}} 08$ & & $06 \underline{Z}_{13}$ \\
\hline$a_{12} 08$ & $06 Z_{12}$ & $a_{12} 08$ & & $06 Z_{12}$ \\
\hline$\underline{a}_{11} 08$ & $06 \underline{Z}_{11}$ & $\underline{\mathbf{a}}_{11} 08$ & & $06 \underline{Z}_{11}$ \\
\hline$a_{10} 00$ & $00 Z_{10}$ & $a_{10} 00$ & & $00 Z_{10}$ \\
\hline$\underline{\mathbf{a}}_{9} \quad 03$ & $02 \underline{Z}_{9}$ & $\underline{\mathbf{a}}_{9} \quad 03$ & \multirow{5}{*}{$20(32) 12$} & $02 \underline{Z}_{9}$ \\
\hline$a_{8} \quad 03$ & $02 Z_{8}$ & $a_{8} \quad 03$ & & $02 Z_{8}$ \\
\hline$\underline{\mathbf{a}}_{7} \quad 05$ & $03 \underline{Z}_{7}$ & $\underline{\mathbf{a}}_{\mathbf{7}} \quad 05$ & & $03 \underline{Z}_{7}$ \\
\hline$a_{6} \quad 05$ & $03 \bar{Z}_{6}$ & $a_{6} \quad 05$ & & $03 \bar{Z}_{6}$ \\
\hline$\underline{a}_{5} \quad 04$ & $02 \underline{Z}_{5}$ & $a_{5} \quad 04$ & & $02 \underline{Z}_{5}$ \\
\hline$a_{4} \quad 04$ & $02 Z_{4}$ & $a_{4} \quad 04$ & & $02 Z_{4}$ \\
\hline$\underline{\mathbf{a}}_{3} \quad 04$ & $01 \underline{Z}_{3}$ & $\underline{\mathbf{a}}_{3} \quad 04$ & & $01 \underline{Z}_{3}$ \\
\hline$a_{2} 03$ & $02 Z_{2}$ & $a_{2} \quad 03$ & $16(2$ & $02 \bar{Z}_{2}$ \\
\hline$\underline{\mathbf{a}}_{\mathbf{1}} 03$ & $02 \underline{Z}_{1}$ & $\underline{\mathbf{a}}_{1} \quad 03$ & & $02 \underline{\mathbf{z}}_{1}$ \\
\hline$a_{0} \quad 02$ & $01 Z_{0}$ & $a_{0} \quad 02$ & & $01 Z_{0}$ \\
\hline Even 33 & (55) 22 & 57 & 52 / 60 & \\
\hline Odd 35 & (57) 22 & 55 & & \\
\hline $\begin{array}{l}08 \\
24 \\
32 \\
\end{array}$ & $\begin{array}{l}06 \\
18 \\
24 \\
\end{array}$ & \multicolumn{3}{|c|}{$\begin{array}{l}14 \text { (well-marked on } 14^{\text {th }} \text { level) } \\
42 \text { (non-marked on } 11-13^{\text {th }} \text { levels) } \\
56\end{array}$} \\
\hline 36 & 20 & \multicolumn{3}{|c|}{56 (well-marked on $00-09^{\text {th }}$ levels) } \\
\hline 68 & 44 & \multicolumn{3}{|c|}{$112(4 \times 28)$ total } \\
\hline 44 & 26 & \multicolumn{3}{|c|}{70 well-marked (with letters) } \\
\hline
\end{tabular}

Tab. 2.1. All primary branches for two species, "A" and "I", with the splitting into $(3 \times 5)$ levels. The pattern $52+62=112$ appears to be the middle case in a specific arithmetical system (Figure 5). Notice that 56 branches are in the upper as well as in the lower part of the Diagram. (Notice the differences between pattern 52 / 60 / 112, valid for all primary branches (in this Table) and the pattern 62 / 59 / 121 (in Table 2.2), valid for secondary branches, where the changes are \pm 10 and \pm 01 . Notice also that the first pattern 52 / 60 / 112 is the middle case within a specific arithmetical system, presented in Figure 5.) 


\begin{tabular}{|c|c|c|c|c|}
\hline$\underline{a}_{9} \quad 08$ & $05 \underline{Z}_{9}$ & âg 08 & & $05 \underline{Z_{9}}$ \\
\hline$a_{8} \quad 09$ & $06 Z_{8}$ & $a_{8} 09$ & & $06 Z_{8}$ \\
\hline$\underline{a}_{7} 13$ & $07 \underline{Z}_{7}$ & $\underline{a}_{7} 13$ & $46(74) 28$ & $07 \underline{Z}_{7}$ \\
\hline$a_{6} \quad 06$ & $04 Z_{6}$ & $a_{6} 06$ & & $04 Z_{6}$ \\
\hline$\underline{a}_{5} 10$ & $06 \underline{\mathbf{Z}}_{5}$ & $\underline{\mathbf{a}}_{5} 10$ & & $06 \underline{Z}_{5}$ \\
\hline$a_{4} \quad 09$ & $04 Z_{4}$ & $a_{4} 09$ & & $04 Z_{4}$ \\
\hline$\underline{\mathbf{a}}_{3} \quad 07$ & $03 \underline{Z}_{3}$ & $\underline{a}_{3} 07$ & & $03 \underline{Z}_{3}$ \\
\hline$a_{2} \quad 05$ & $04 Z_{2}$ & $a_{2} 05$ & 31 & $04 Z_{2}$ \\
\hline$\underline{\mathbf{a}}_{\mathbf{1}} 06$ & $01 \underline{Z}_{1}$ & $\underline{\mathbf{a}}_{\mathbf{1}} 06$ & & $01 \underline{Z}_{1}$ \\
\hline$a_{0} \quad 04$ & $04 Z_{0}$ & $a_{0} 04$ & & $04 Z_{0}$ \\
\hline $\begin{array}{l}\text { Odd } 44 \\
\text { Even } 33\end{array}$ & $\begin{array}{l}\text { (66) } 22 \\
(55) 22\end{array}$ & $\begin{array}{l}(55) \\
(66)\end{array}$ & 62 / 59 & \\
\hline 77 & (11) 44 & \multicolumn{3}{|c|}{$121(56+65)$} \\
\hline \multicolumn{5}{|c|}{ Middle pair $60 / 61$ vs $62 / 59$ as result } \\
\hline
\end{tabular}

Table 2.2. All secondary branches for two species, "A" and "I", with the splitting into $(5+5)$ levels. There are none of them after the 9th level. [Cf. pattern 74/77 with the pattern 64/66 in Table 4.1; then 44/46 with the pattern 64/66 also in Table 4.1.] 


\begin{tabular}{|c|c|c|c|c|}
\hline a 903 & $02 \underline{Z}_{9}$ & $\underline{a}_{9} \quad 03$ & & $02 \underline{Z_{9}}$ \\
\hline$a_{8} \quad 03$ & $02 Z_{8}$ & $a_{8} 03$ & & $02 Z_{8}$ \\
\hline$\underline{\mathbf{a}}_{7} 05$ & $03 \underline{Z}_{7}$ & $\underline{\mathbf{a}}_{7} \quad 05$ & & $03 \underline{Z}_{7}$ \\
\hline$a_{6} \quad 04$ & $02 Z_{6}$ & $a_{6} \quad 04$ & & $02 Z_{6}$ \\
\hline$\underline{\underline{a}}_{5} \quad 03$ & $02 \underline{Z}_{5}$ & $\underline{a}_{5} \quad 03$ & & $02 \underline{Z}_{5}$ \\
\hline$a_{4} 03$ & $01 Z_{4}$ & $a_{4} \quad 03$ & & $01 Z_{4}$ \\
\hline$\underline{a}_{3} 03$ & $01 \underline{Z}_{3}$ & $\underline{\mathbf{a}}_{\mathbf{3}} 03$ & & $01 \underline{Z}_{3}$ \\
\hline$a_{2} \quad 02$ & $02 \bar{Z}_{2}$ & $a_{2} \quad 02$ & $11(17) 06$ & $02 Z_{2}$ \\
\hline$\underline{\mathbf{a}}_{\mathbf{1}} 02$ & $01 \underline{\mathbf{z}}_{1}$ & $\underline{a}_{1} \quad 02$ & & $01 \underline{Z}_{1}$ \\
\hline$a_{0} \quad 01$ & $01 \bar{Z}_{0}$ & $a_{0} \quad 01$ & & $01 \bar{Z}_{0}$ \\
\hline $\begin{array}{l}\text { Odd } 16 \\
\text { Even } 13\end{array}$ & $\begin{array}{ll}(25) & 09 \\
(21) & 08\end{array}$ & $\begin{array}{l}(22) \\
(24)\end{array}$ & $24 / 22$ & \\
\hline 29 & 17 & 46 & 46 & \\
\hline \multicolumn{5}{|c|}{$46+10=56$} \\
\hline
\end{tabular}

Tab. 3.1. All nodes for two species, "A" and "I", with the splitting into (5+5) levels. The balances are self-evident. [Notice a special balance: 46 nodes +10 branchings (Tables 3.1 and 3.2) equals 56 group tree-entities in correspondence with 56 primary branches (Table 1.1) as individual tree-entities.] 


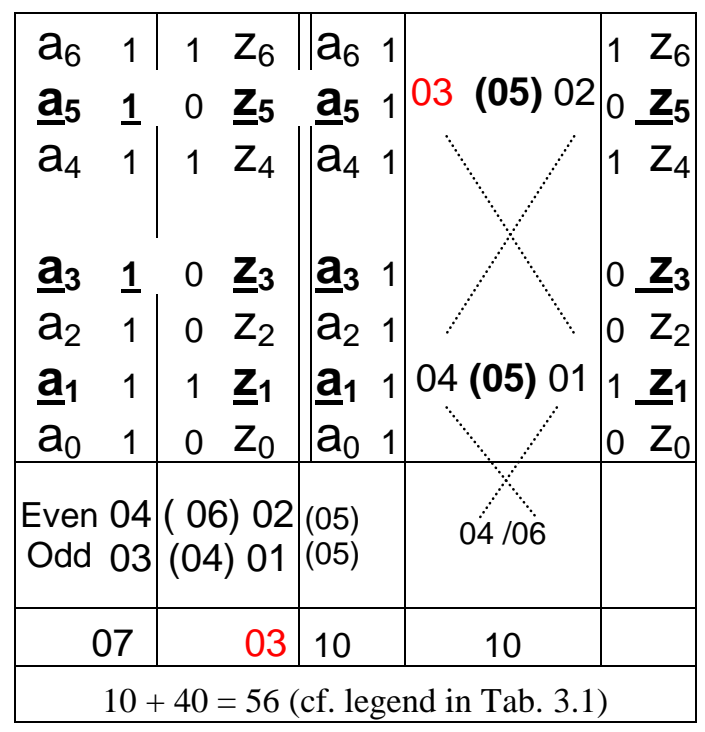

Tab. 3.2. All branchings for two species, "A" and "I", with the splitting into (4+3) levels. This is due to the fact that there are branchings in the Diagram just from the zeroth to the 6th level. This finding requires that in the analysis of the number of all branches, except for splitting into the (5+5) levels as in Table 4.1, we must as well analyze the splitting into $(7+3)$ levels as in Table 4.2 , and then into $(3+4+3)$ as in Table 4.3 and $(3+2+2+3)$ as in Table 4.4. The balances are self-evident. [Notice that the left tree of the Diagram (Figure 1.1) contains two large branches; and on the left branch there are only two branchings (bold, underlined units in the second column).] 


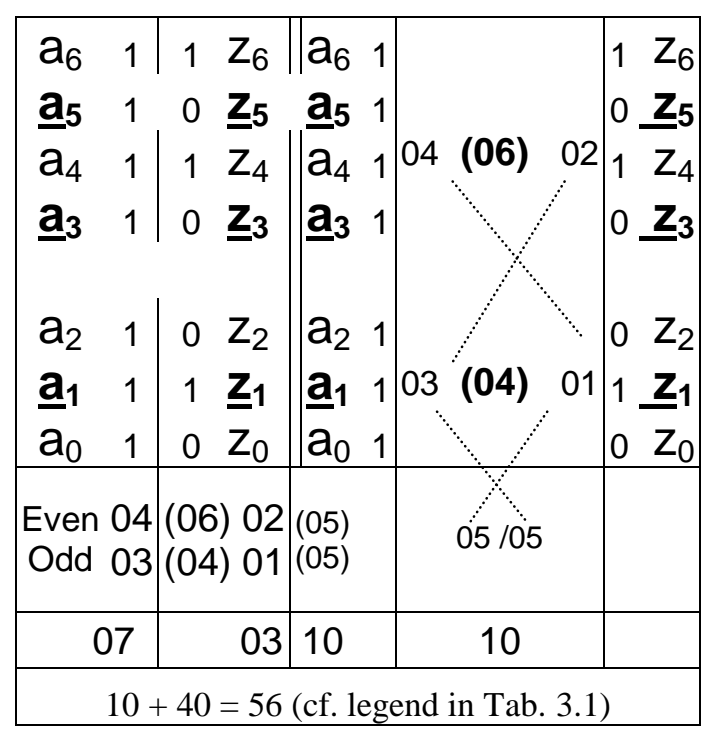

Tab. 3.3. All branchings for two species, "A" and "I", with the splitting into (3+4) levels as a reverse way in relation to Table 3.2. Notice that the splitting of 7 levels into 3 and $4(3+4=7)$ represent a correspondence with the Lucas numbers series at the same time (Figure D.1). 


\begin{tabular}{|c|c|c|c|c|}
\hline $\begin{array}{ll}\underline{\mathbf{a}}_{9} & 11\end{array}$ & $07 \underline{Z}_{9}$ & $\underline{a}_{9} 11$ & \multirow{5}{*}{66 (106) 40} & $07 \underline{Z_{9}}$ \\
\hline$a_{8} \quad 12$ & $08 Z_{8}$ & $a_{8} 12$ & & $08 Z_{8}$ \\
\hline $\begin{array}{ll}\underline{\mathbf{a}}_{7} & 18\end{array}$ & $10 \underline{\mathbf{Z}}_{7}$ & $\underline{\mathbf{a}}_{7} 18$ & & $10 \underline{\mathbf{Z}}_{7}$ \\
\hline$a_{6} \quad 11$ & $07 Z_{6}$ & $a_{6} 11$ & & $07 Z_{6}$ \\
\hline $\begin{array}{ll}\underline{a}_{5} & 14\end{array}$ & $08 \underline{Z}_{5}$ & $\underline{\mathbf{a}}_{5} 14$ & & $08 \underline{\mathbf{Z}}_{5}$ \\
\hline$a_{4} 13$ & $06 Z_{4}$ & $a_{4} 13$ & & $06 Z_{4}$ \\
\hline$\underline{\mathbf{a}}_{3} \quad 11$ & $04 \underline{Z}_{3}$ & $\underline{\mathbf{a}}_{3} 11$ & & $04 \underline{Z}_{3}$ \\
\hline$a_{2} \quad 08$ & $06 Z_{2}$ & $a_{2} 08$ & $47(71) 24$ & $06 Z_{2}$ \\
\hline$\underline{\mathbf{a}}_{\mathbf{1}} \quad 09$ & $03 \underline{Z}_{1}$ & $\underline{\mathbf{a}}_{\mathbf{1}} 09$ & & $03 \underline{Z}_{1}$ \\
\hline$a_{0} \quad 06$ & $05 Z_{0}$ & $a_{0} 06$ & & $05 Z_{0}$ \\
\hline $\begin{array}{l}\text { Odd } 63 \\
\text { Even } 50\end{array}$ & $\begin{array}{l}(95) 32 \\
(82) 32\end{array}$ & $\begin{array}{l}(82) \\
(95)\end{array}$ & $90 / 87$ & \\
\hline 113 & 64 & 177 & 177 & \\
\hline$(177=$ & 00.0 & 000 & & \\
\hline
\end{tabular}

Tab. 4.1. All branches (primary + secondary) for two species, "A" and "I", with the splitting into (5+5) levels. The pattern 90/87 appears to be an inverse result 80/97 which appears by the splitting into $(7+3)$ levels (Table 4.2) and a strict balance in relation to $89 / 88$ (the balance in frame of \pm 1 ) by the splitting into (3+4+3) levels (Table 4.3). [Cf. pattern 64/66 with pattern 74/77 and pattern 44/46 in Table 2.2.] 


\begin{tabular}{|c|c|c|c|c|}
\hline $\begin{array}{ll}\underline{\mathbf{a}}_{9} & 11 \\
\mathbf{a}_{8} & 12 \\
\underline{\mathbf{a}}_{7} & 18\end{array}$ & $\begin{array}{ll}07 & \underline{Z}_{9} \\
08 & Z_{8} \\
10 & \underline{Z}_{7}\end{array}$ & $\begin{array}{ll}\underline{a}_{9} & 11 \\
\mathbf{a}_{8} & 12 \\
\underline{a}_{7} & 18\end{array}$ & $41(66) 25$ & $\begin{array}{lll}07 & \underline{Z}_{9} \\
08 & Z_{8} \\
10 & \underline{Z}_{7}\end{array}$ \\
\hline$a_{6} 11$ & $07 Z_{6}$ & $a_{6} 11$ & & $07 Z_{6}$ \\
\hline$\underline{a}_{5} 14$ & $08 \mathbf{Z}_{5}$ & $\underline{a}_{5} 14$ & & $08 \underline{Z}_{5}$ \\
\hline$a_{4} 13$ & $06 Z_{4}$ & $a_{4} 13$ & & $06 Z_{4}$ \\
\hline$\underline{a}_{3} 11$ & $04 \underline{Z}_{3}$ & $\underline{a}_{3} 11$ & 72 (111) 39 & $04 \underline{Z}_{3}$ \\
\hline$a_{2} 08$ & $06 Z_{2}$ & $a_{2} 08$ & & $06 Z_{2}$ \\
\hline$\underline{\mathbf{a}}_{\mathbf{1}} 09$ & $03 \underline{Z}_{1}$ & $\underline{a}_{\mathbf{1}} 09$ & & $03 \underline{Z}_{1}$ \\
\hline$a_{0} \quad 06$ & $05 Z_{0}$ & $a_{0} 06$ & & $05 Z_{0}$ \\
\hline $\begin{array}{l}\text { Odd } 63 \\
\text { Even } 50\end{array}$ & $\mid \begin{array}{l}\text { ( 95) } 32 \\
(82)\end{array} 32$ & $\begin{array}{l}82 \\
95\end{array}$ & $80 / 97$ & \\
\hline 113 & 64 & 177 & 177 & \\
\hline \multicolumn{5}{|c|}{ 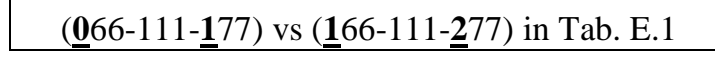 } \\
\hline
\end{tabular}

Tab. 4.2. All branches (primary + secondary) for two species, "A" and "I", with the splitting into (7+3) levels with pattern 80/97 corresponding to the pattern 90/87 which appears by the splitting into (5+5) levels in Table 4.1. On the other hand pattern ㅁ66-111-1777 corresponds to pattern $\underline{\mathbf{1}} 66-111-\underline{\mathbf{2}} 77$ in genetic code (Appendix E). All other balances are self-evident. 


\begin{tabular}{|c|c|c|c|c|}
\hline $\begin{array}{ll}\underline{a}_{9} & 11\end{array}$ & $07 \underline{Z}_{9}$ & $a_{9} 11$ & & $07 \underline{Z}$ \\
\hline $\begin{array}{ll}\bar{a}_{8} & 12\end{array}$ & $08 \bar{Z}_{8}$ & $\bar{a}_{8} 12$ & $41(66)$ & $08 \bar{Z}$ \\
\hline$\underline{\mathbf{a}}_{7} 18$ & $10 \underline{Z}_{7}$ & $\underline{a}_{7} 18$ & & $10 \underline{Z}$ \\
\hline$a_{6} 11$ & $07 Z_{6}$ & $a_{6} 11$ & & $07 \mathrm{Z}$ \\
\hline$\underline{a}_{5} \quad 14$ & $08 \underline{Z}_{5}$ & $a_{\mathbf{5}} 14$ & $49(74$ & $08 \underline{Z}$ \\
\hline$a_{4}$ & $06^{Z_{4}}$ & $a_{4}$ & & $06^{Z}$ \\
\hline$\underline{\mathbf{a}}_{3} \quad 11$ & $04 \underline{Z}_{3}$ & $\underline{a}_{3} 11$ & & 04 Z \\
\hline$a_{2} \quad 08$ & $06 Z_{2}$ & $a_{2} 08$ & & $06 Z$ \\
\hline $\begin{array}{ll}\underline{\mathbf{a}}_{\mathbf{1}} & 09\end{array}$ & $03 \underline{Z}_{1}$ & $\underline{a}_{1} 09$ & $23(37) 14$ & $03 \underline{Z}$ \\
\hline$a_{0} \quad 06$ & $05 Z_{0}$ & $a_{0} 06$ & & $05 Z$ \\
\hline $\begin{array}{l}\text { Odd } 63 \\
\text { Even } 50\end{array}$ & $\begin{array}{l}(95) 32 \\
(82) 32\end{array}$ & $\begin{array}{l}82 \\
95\end{array}$ & $89 / 88$ & \\
\hline 113 & 64 & 177 & 177 & \\
\hline \multicolumn{5}{|c|}{$37+74=111$} \\
\hline
\end{tabular}

Tab. 4.3. This Table follows from Table 4.2. The formal splitting into $(3+4+3)$ levels corresponds to an extended Cantor triadic set (Figure D.2). On the other hand, the number of the branches follows from the splitting of the first Shcherbak's quantum of "the same symbols" (111 in previous Table) into two quantums "arranged by the cyclic permutation" $(037+074)$ where the quantum 037 is the "Prime quantum 037"; all these quantums in relation to number 66, and altogether in connection with a specific and unique arithmetical system (Table B.1 and Survey B.1 in Appendix B). 


\begin{tabular}{|c|c|c|c|c|c|}
\hline$\underline{\mathbf{a}_{9}}$ & 11 & $07 \underline{Z}_{9}$ & $\underline{a}_{9} 11$ & & $07 \underline{Z}_{9}$ \\
\hline$a_{8}$ & 12 & $08 Z_{8}$ & $a_{8} 12$ & $41(66) 25$ & $08 Z_{8}$ \\
\hline$\underline{a}_{7}$ & 18 & $10 \underline{Z}_{7}$ & $\underline{a}_{7} 18$ & & $10 \underline{Z}_{7}$ \\
\hline$a_{6}$ & 11 & $07 Z_{6}$ & $a_{611}$ & $25(40)$ & $07 Z_{6}$ \\
\hline$\underline{a}_{5}$ & 14 & $08 \underline{Z}_{5}$ & $\underline{a}_{5} 14$ & & $08 \underline{Z}_{5}$ \\
\hline$a_{4}$ & 13 & $06 Z_{4}$ & $a_{4} 13$ & & $06 Z_{4}$ \\
\hline$\underline{\mathbf{a}}_{3}$ & 11 & $04 \underline{Z}_{3}$ & $\underline{a}_{3} 11$ & & $04 \underline{Z}_{3}$ \\
\hline$a_{2}$ & 08 & $06 Z_{2}$ & $a_{2} 08$ & & $06 Z_{2}$ \\
\hline$\underline{\mathbf{a}}_{1}$ & 09 & $03 \underline{z}_{1}$ & $\underline{\mathbf{a}}_{\mathbf{1}} \quad 09$ & $23(37) 14$ & $03 \underline{Z}_{1}$ \\
\hline$a_{0}$ & 06 & $05 Z_{0}$ & $a_{0} 06$ & & $05 Z_{0}$ \\
\hline & $\begin{array}{l}63 \\
50\end{array}$ & $\mid \begin{array}{ll}(95) & 32 \\
(82) & 32\end{array}$ & $\begin{array}{l}82 \\
95\end{array}$ & $94 / 83$ & \\
\hline & 113 & (13) 64 & 177 & 177 & \\
\hline \multicolumn{6}{|c|}{$(94 / 83$ vs $82 / 95)(94-83=11)$} \\
\hline
\end{tabular}

Tab. 4.4. All branches (primary + secondary) for two species, "A" and "I", with the splitting into $(3+2+2+3)$ levels. The balances are self-evident. 


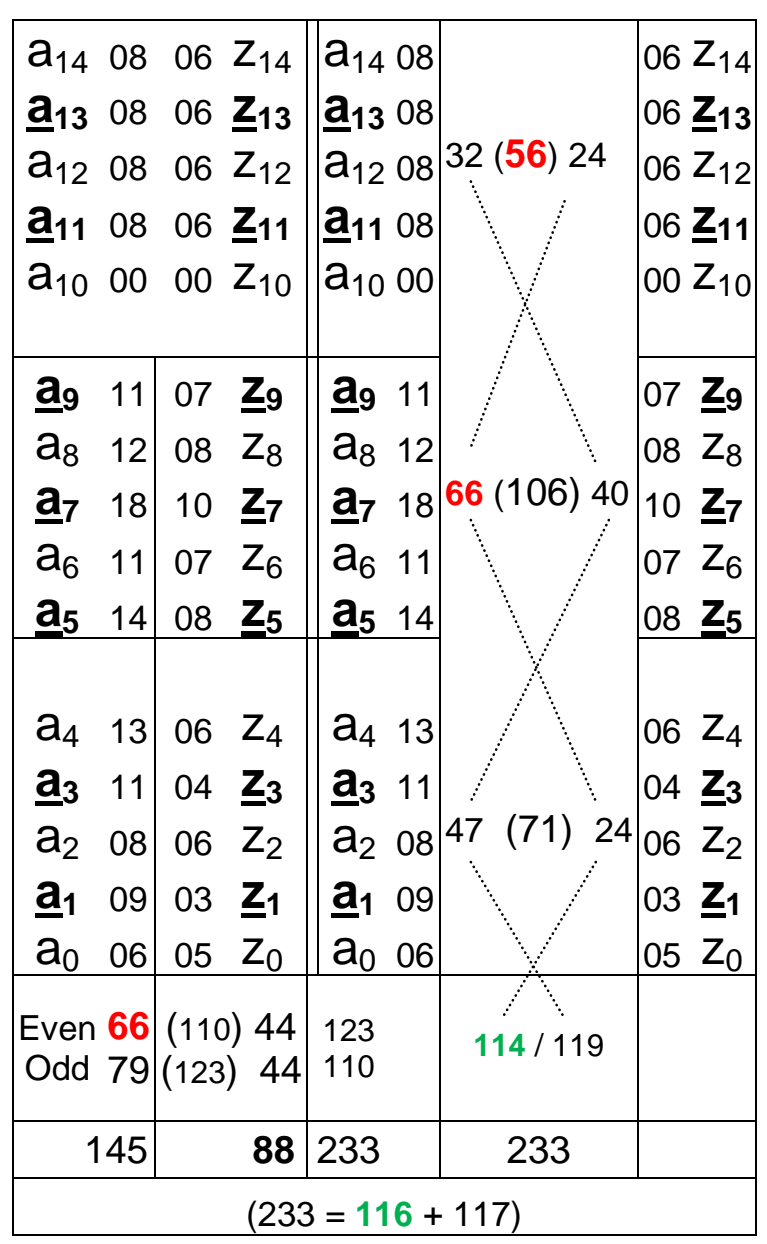

Tab. 4.5. All branches (primary + secondary) for two species, "A" and "I", with the splitting into ( $3 \times$ 5) levels, $0-14$. The balances are self-evident. 


\begin{tabular}{|c|c|c|c|}
\hline \multicolumn{2}{|c|}{ Primary } & \multicolumn{2}{|c|}{ Secondary } \\
\hline B 00 & $06 \mathrm{G}$ & B 01 & $01 \mathrm{G}$ \\
\hline C 01 & $02 \mathrm{H}$ & C 01 & $01 \mathrm{H}$ \\
\hline D 02 & $00 \mathrm{~K}$ & D 01 & $01 \mathrm{~K}$ \\
\hline E 10 & $01 \mathrm{~L}$ & E 00 & $01 \mathrm{~L}$ \\
\hline F 14 & & $F 00$ & \\
\hline \multicolumn{2}{|c|}{$27 \mid 09$} & \multicolumn{2}{|c|}{03|| 04} \\
\hline & 36 & 43) & 07 \\
\hline \multicolumn{4}{|c|}{$\begin{array}{l}(233+43=276) \\
(276+56=332)\end{array}$} \\
\hline \multicolumn{4}{|c|}{99} \\
\hline & $6=216$ & $n+6$ & $50^{\text {up }}$ \\
\hline
\end{tabular}

Table 5. All branches (primary + secondary) for "other nine species" for the left and the right part of the Diagram, at all 15 levels. The equation $27+09=36$ appears to be a special Darwin's equation, valid to determination of the genetic code (Figure $6,7 \& 8$ and Table 6.1); and the equation $03+04=07$ corresponds to the first three members of Lucas number series (Figure D.1).The number 233 comes from Table 4.5 and together with this result (43) makes 276 which is the total number of branches within the Diagram. In addition: $56=46$ nodes plus 10 branchings, and from that all "branch" entities/quantities equal 332 as a mirror pattern of the 233 . 


\begin{tabular}{|c|c|c|c|c|}
\hline $\begin{array}{ll}\hat{a}_{14} & 01 \\
\hat{a}_{13} & 01 \\
\hat{a}_{12} & 01 \\
\hat{a}_{11} & 01 \\
\hat{a}_{10} & 00\end{array}$ & $\begin{array}{ll}00 & \hat{Z}_{14} \\
00 & \hat{z}_{13} \\
00 & \hat{Z}_{12} \\
00 & \hat{z}_{11} \\
00 & \hat{Z}_{10}\end{array}$ & $\mid \begin{array}{ll}\hat{a}_{14} & 01 \\
\hat{a}_{13} & 01 \\
\hat{a}_{12} & 01 \\
\hat{a}_{11} & 01 \\
\hat{a}_{10} & 00\end{array}$ & $04(04) 00$ & $\begin{array}{ll}00 & \hat{Z}_{14} \\
00 & \hat{z}_{13} \\
00 & \hat{Z}_{12} \\
00 & \hat{\mathbf{z}}_{11} \\
00 & \hat{\mathbf{Z}}_{10}\end{array}$ \\
\hline $\begin{array}{ll}\hat{a ̂}_{9} & 02 \\
\hat{a}_{8} & 02 \\
\hat{a}_{7} & 02 \\
\hat{a}_{6} & 02 \\
\hat{a}_{5} & 02 \\
\end{array}$ & $\begin{array}{ll}00 & \hat{\underline{Z}}_{9} \\
00 & \hat{Z}_{8} \\
00 & \hat{\mathbf{Z}}_{7} \\
00 & \hat{\mathbf{Z}}_{6} \\
01 & \hat{\underline{Z}}_{5}\end{array}$ & $\begin{array}{ll}\hat{a ̂}_{9} & 02 \\
\hat{a}_{8} & 02 \\
\hat{a}_{7} & 02 \\
\hat{a}_{6} & 02 \\
\hat{a}_{5} & 02 \\
\end{array}$ & 10 & $\begin{array}{ll}00 & \hat{\mathbf{Z}}_{9} \\
00 & \hat{\mathbf{Z}}_{8} \\
00 & \hat{\mathbf{z}}_{7} \\
00 & \hat{\mathbf{Z}}_{6} \\
01 & \hat{\mathbf{Z}}_{5} \\
\end{array}$ \\
\hline $\begin{array}{ll}\hat{a}_{4} & 02 \\
\hat{a}_{3} & 02 \\
\hat{a}_{2} & 02 \\
\hat{a}_{1} & 03 \\
\hat{a}_{0} & 04 \\
\end{array}$ & $\begin{array}{ll}01 & \hat{Z}_{4} \\
01 & \hat{Z}_{3} \\
01 & \hat{Z}_{2} \\
02 & \hat{\mathbf{Z}}_{1} \\
03 & \hat{Z}_{0}\end{array}$ & $\begin{array}{ll}\hat{a}_{4} & 02 \\
\hat{a}_{3} & 02 \\
\hat{a}_{2} & 02 \\
\hat{a}_{1} & 03 \\
\hat{a}_{0} & 04 \\
\end{array}$ & $13(21$ & $\begin{array}{ll}01 & \hat{Z}_{4} \\
01 & \hat{\mathbf{Z}}_{3} \\
01 & \hat{Z}_{2} \\
02 & \hat{\underline{Z}}_{1} \\
03 & \hat{Z}_{0} \\
\end{array}$ \\
\hline $\begin{array}{l}\text { Odd } 13 \\
\text { Even } 14\end{array}$ & $\begin{array}{l}(17) 04 \\
\text { (19) } 05\end{array}$ & $\begin{array}{l}(18) \\
(18)\end{array}$ & $18 / 18$ & \\
\hline $\begin{array}{l}01 \\
03 \\
04 \\
\end{array}$ & $\begin{array}{l}00 \\
00 \\
00\end{array}$ & \multicolumn{3}{|c|}{$\begin{array}{l}01 \text { (well-marked on } 14^{\text {th }} \text { level) } \\
03 \text { (non-marked on } 11-13^{\text {th }} \text { levels) } \\
04\end{array}$} \\
\hline 02 & 00 & \multicolumn{3}{|c|}{02 (well-marked on $0-9^{\text {th }}$ levels) } \\
\hline 21 & 09 & \multicolumn{3}{|c|}{30 (non-marked on $0-9^{\text {th }}$ levels) } \\
\hline 24 & 09 & \multicolumn{3}{|c|}{33 (non-marked on $0-14^{\text {th }}$ levels) } \\
\hline 27 & & \multicolumn{3}{|c|}{36 total } \\
\hline \multicolumn{5}{|c|}{$(18=28-10)$} \\
\hline
\end{tabular}

Table 6.1. All primary branches for 9 species (B, C, D, E, F on the left and G, H, K, L on the right) at 014 levels. The final result is the Darwin's equation $(27+09=36)$ (cf. Figures $6 \& 7)$. 


\begin{tabular}{|c|c|c|c|c|}
\hline $\begin{array}{ll}\hat{a ̂}_{9} & 02 \\
\hat{a}_{8} & 02 \\
\hat{a}_{7} & 02 \\
\hat{\underline{a}}_{6} & 02 \\
\hat{a}_{5} & 02\end{array}$ & $\begin{array}{ll}00 & \underline{\mathbf{Z}}_{9} \\
00 & \hat{\mathbf{Z}}_{8} \\
00 & \hat{\underline{Z}}_{7} \\
00 & \hat{\mathbf{Z}}_{6} \\
01 & \underline{\underline{Z}}_{5}\end{array}$ & $\begin{array}{ll}\underline{a}_{9} & 02 \\
\hat{a}_{8} & 02 \\
\hat{a}_{7} & 02 \\
\hat{a}_{6} & 02 \\
\underline{\hat{a}}_{5} & 02 \\
\end{array}$ & $10(11)$ & $\mid \begin{array}{ll}00 & \hat{\mathbf{Z}}_{9} \\
00 & \hat{\mathbf{Z}}_{8} \\
00 & \hat{\mathbf{Z}}_{7} \\
00 & \hat{\mathbf{Z}}_{6} \\
01 & \underline{\mathbf{Z}}_{5}\end{array}$ \\
\hline $\begin{array}{ll}\hat{a}_{4} & 02 \\
\hat{a}_{3} & 02 \\
\underline{\hat{a}}_{2} & 02 \\
\hat{a}_{1} & 03 \\
\underline{\hat{a}}_{0} & 04\end{array}$ & $\begin{array}{ll}01 & \hat{Z}_{4} \\
01 & \hat{\underline{Z}}_{3} \\
01 & \hat{Z}_{2} \\
02 & \hat{\underline{Z}}_{1} \\
03 & \hat{Z}_{0}\end{array}$ & $\left|\begin{array}{ll}\hat{a}_{4} & 02 \\
\hat{a}_{3} & 02 \\
\hat{a}_{2} & 02 \\
\hat{a}_{1} & 03 \\
\hat{a}_{0} & 04\end{array}\right|$ & & $\mid \begin{array}{ll}01 & \hat{Z}_{4} \\
01 & \hat{Z}_{3} \\
01 & \hat{Z}_{2} \\
02 & \hat{Z}_{1} \\
03 & \hat{Z}_{0}\end{array}$ \\
\hline $\begin{array}{l}\text { Odd } 11 \\
\text { Even } 12\end{array}$ & $\begin{array}{l}\text { (15) } 04 \\
\text { (17) } 05\end{array}$ & $\begin{array}{l}(16) \\
(16)\end{array}$ & $18 / 14$ & \\
\hline 23 & 09 & 32 & 32 & \\
\hline \multicolumn{5}{|c|}{$56+32=88$} \\
\hline
\end{tabular}

Table 6.2. Primary branches for 9 species (B, C, D, E, F on the left and G, H, K, L on the right) at 0-9 levels. 


\begin{tabular}{|c|c|c|c|c|}
\hline $\begin{array}{ll}\hat{a}_{9} & 00 \\
\hat{a}_{8} & 00 \\
\hat{a}_{7} & 00 \\
\hat{\underline{a}}_{6} & 00 \\
\hat{a}_{5} & 00\end{array}$ & $\begin{array}{ll}00 & \hat{Z}_{9} \\
00 & \hat{Z}_{8} \\
00 & \hat{\mathbf{Z}}_{7} \\
01 & \hat{Z}_{6} \\
00 & \hat{\mathbf{Z}}_{5}\end{array}$ & $\begin{array}{ll}\hat{a}_{9} & 00 \\
\hat{a}_{8} & 00 \\
\hat{a}_{7} & 00 \\
\hat{a}_{6} & 00 \\
\underline{\underline{a}}_{5} & 00\end{array}$ & 1 & $\begin{array}{ll}00 & \hat{\mathbf{Z}}_{9} \\
00 & \hat{Z}_{8} \\
00 & \underline{\mathbf{Z}}_{7} \\
01 & \hat{Z}_{6} \\
00 & \underline{\underline{Z}}_{5}\end{array}$ \\
\hline $\begin{array}{ll}\hat{a}_{4} & 00 \\
\hat{a}_{3} & 00 \\
\underline{\hat{a}}_{2} & 01 \\
\hat{a}_{1} & 01 \\
\hat{a}_{0} & 01\end{array}$ & $\begin{array}{ll}00 & \hat{Z}_{4} \\
00 & \hat{\mathbf{Z}}_{3} \\
01 & \hat{Z}_{2} \\
01 & \hat{\mathbf{Z}}_{1} \\
01 & \hat{Z}_{0}\end{array}$ & $\begin{array}{ll}\hat{a}_{4} & 00 \\
\hat{a}_{3} & 00 \\
\hat{a}_{2} & 01 \\
\hat{a}_{1} & 01 \\
\hat{a}_{0} & 01\end{array} \mid$ & & $3 \begin{array}{l}00 \hat{\mathbf{Z}} \\
00 \hat{\mathbf{Z}} \\
01 \hat{\mathbf{Z}} \\
01 \hat{\mathbf{Z}} \\
01 \hat{\mathbf{Z}}\end{array}$ \\
\hline $\begin{array}{l}\text { Odd } 01 \\
\text { Even } 02\end{array}$ & $\begin{array}{l}(02) \\
(05)\end{array} 03$ & $\begin{array}{l}03 \\
04\end{array}$ & $03 / 04$ & \\
\hline 03 & 04 & 07 & 07 & \\
\hline
\end{tabular}

Table 6.3. All secondary branches for 9 species (B, C, D, E, F on the left and G, H, K, L on the right) at 0-9 levels. 


\begin{tabular}{|c|c|c|c|c|}
\hline$\hat{a}_{14} 01$ & $00 \hat{Z}_{14}$ & $\hat{a}_{1401}$ & & $00 \hat{Z}_{1}$ \\
\hline ấ$_{13} 01$ & $00 \underline{\mathbf{z}}_{13}$ & $\underline{\underline{a}}_{13} 01$ & & $00 \underline{\mathbf{z}}_{1}$ \\
\hline$\hat{\mathrm{a}}_{12} 01$ & $00 \hat{Z}_{12}$ & $\overline{\mathrm{a}}_{1201} 01$ & $04(04) 00$ & $000 \bar{Z}_{1}$ \\
\hline $\begin{array}{ll}\underline{\mathbf{a}}_{11} & 01\end{array}$ & $00 \underline{\underline{\mathbf{z}}}_{11}$ & $\underline{\underline{a}}_{11} 01$ & & $00 \underline{\underline{\mathbf{z}}}_{1}$ \\
\hline$\hat{a}_{10} \quad 00$ & $00 \hat{Z}_{10}$ & $\hat{a}_{10} 00$ & & $00 \hat{z}_{1 c}$ \\
\hline $\begin{array}{ll}\hat{\mathrm{a}}_{9} & 02 \\
\hat{\mathrm{a}}_{8} & 02 \\
\hat{\mathrm{a}}_{7} & 02 \\
\hat{\mathrm{a}}_{6} & 02 \\
\hat{\mathrm{a}}_{5} & 02\end{array}$ & $\begin{array}{ll}00 & \hat{Z}_{9} \\
00 & \hat{Z}_{8} \\
00 & \hat{\mathbf{Z}}_{7} \\
01 & \hat{Z}_{6} \\
01 & \hat{\mathbf{Z}}_{5}\end{array}$ & $\begin{array}{ll}\underline{\mathbf{a}}_{9} & 02 \\
\hat{a}_{8} & 02 \\
\hat{a}_{7} & 02 \\
\hat{a}_{6} & 02 \\
\hat{\mathbf{a}}_{5} & 02 \\
\end{array}$ & & $\begin{array}{ll}00 & \hat{\mathbf{Z}}_{9} \\
00 & \hat{\mathbf{Z}}_{8} \\
00 & \underline{\mathbf{Z}}_{7} \\
01 & \hat{\mathbf{Z}}_{6} \\
01 & \underline{\mathbf{Z}}_{5}\end{array}$ \\
\hline $\begin{array}{ll}\hat{a}_{4} & 02 \\
\hat{a}_{3} & 02 \\
\hat{a}_{2} & 03 \\
\hat{a}_{1} & 04 \\
\hat{a}_{1} & 0 \\
\hat{a}_{0} & 05\end{array}$ & $\begin{array}{ll}01 & \hat{Z}_{4} \\
01 & \hat{\mathbf{Z}}_{3} \\
02 & \hat{Z}_{2} \\
03 & \hat{\mathbf{z}}_{1} \\
04 & \hat{\mathbf{Z}}_{0}\end{array}$ & $\begin{array}{ll}\hat{\mathrm{a}}_{4} & 02 \\
\hat{\mathbf{a}}_{3} & 02 \\
\hat{\mathrm{a}}_{2} & 03 \\
\hat{\mathrm{a}}_{1} & 04 \\
\hat{\mathrm{a}}_{0} & 05 \\
\end{array}$ & & $\begin{array}{ll}01 & \hat{Z}_{4} \\
01 & \hat{\mathbf{Z}}_{3} \\
02 & \hat{Z}_{2} \\
03 & \hat{\mathbf{Z}}_{1} \\
04 & \hat{Z}_{0}\end{array}$ \\
\hline $\begin{array}{ll}\text { Even } & 16 \\
\text { Odd } & 14\end{array}$ & $\begin{array}{ll}\text { (24) } & 08 \\
\text { (19) } & 05\end{array}$ & $\begin{array}{l}(22) \\
(21)\end{array}$ & $21 / 22$ & \\
\hline 30 & 13 & 43 & 43 & \\
\hline
\end{tabular}

Table 6.4. All branches (primary + secondary) for 9 species (B, C, D, E, F on the left and G, H, K, L on the right) at $0-14$ levels. Notice the balances: $21 / 22$ versus $19 / 24$ as a change for \pm 2 ; then: 27 as 9 × 3 and 30 as $10 \times 3$. 


\begin{tabular}{|c|c|c|c|c|}
\hline $\begin{array}{ll}\hat{a ̂}_{9} & 02 \\
\hat{a}_{8} & 02 \\
\hat{a}_{7} & 02 \\
\hat{\underline{a}}_{6} & 02 \\
\hat{a}_{5} & 02\end{array}$ & $\begin{array}{ll}00 & \hat{\mathbf{Z}}_{9} \\
00 & \hat{\mathbf{Z}}_{8} \\
00 & \hat{\underline{Z}}_{7} \\
01 & \hat{\mathbf{Z}}_{6} \\
01 & \underline{\underline{Z}}_{5}\end{array}$ & $\mid \begin{array}{ll}\hat{a}_{9} & 02 \\
\hat{a}_{8} & 02 \\
\hat{\mathbf{a}}_{7} & 02 \\
\hat{a}_{6} & 02 \\
\hat{\underline{a}}_{5} & 02 \\
\end{array}$ & $10(12)$ & $2=\begin{array}{l}00 \hat{\mathbf{Z}}_{9} \\
00 \hat{Z}_{8} \\
00 \hat{\underline{Z}}_{7} \\
01 \hat{\mathbf{Z}}_{6} \\
01 \underline{\mathbf{z}}_{5}\end{array}$ \\
\hline $\begin{array}{ll}\hat{a}_{4} & 02 \\
\hat{a}_{3} & 02 \\
\underline{\hat{a}}_{2} & 03 \\
\hat{a}_{1} & 04 \\
\underline{\hat{a}}_{0} & 05\end{array}$ & $\begin{array}{ll}01 & \hat{Z}_{4} \\
01 & \hat{z}_{3} \\
02 & \hat{Z}_{2} \\
03 & \hat{\underline{z}}_{1} \\
04 & \hat{Z}_{0}\end{array}$ & $\mid \begin{array}{ll}\hat{a}_{4} & 02 \\
\hat{\mathbf{a}}_{3} & 02 \\
\hat{a}_{2} & 03 \\
\hat{\mathbf{a}}_{1} & 04 \\
\hat{\mathbf{a}}_{0} & 05 \\
\end{array}$ & & $\mid \begin{array}{ll}01 & \hat{Z}_{4} \\
01 & \underline{\underline{Z}}_{3} \\
02 & \hat{Z}_{2} \\
03 & \underline{\underline{Z}}_{1} \\
04 & \hat{Z}_{0}\end{array}$ \\
\hline $\begin{array}{l}\text { Odd } 12 \\
\text { Even } 14\end{array}$ & $\begin{array}{l}\text { (17) } 05 \\
\text { (22) } 08\end{array}$ & $\left(\begin{array}{l}(19) \\
(20)\end{array}\right.$ & $21 / 18$ & \\
\hline 26 & 13 & 39 & 39 & \\
\hline \multicolumn{5}{|c|}{ The sums: $17,18,19,20,21,22$} \\
\hline
\end{tabular}

Table 6.5. All branches (primary + secondary) for 9 species (B, C, D, E, F on the left and G, H, K, L on the right) at 0-9 levels. 


\begin{tabular}{|c|c|c|c|c|}
\hline $\begin{array}{ll}\hat{a}_{14} & 09 \\
\hat{a}_{13} & 09 \\
\hat{a}_{12} & 09 \\
\hat{a}_{11} & 09 \\
\hat{a}_{10} & 00\end{array}$ & $\begin{array}{ll}06 & \hat{\mathbf{Z}}_{14} \\
06 & \hat{\mathbf{Z}}_{13} \\
06 & \hat{\mathbf{Z}}_{12} \\
06 & \hat{\mathbf{Z}}_{11} \\
00 & \hat{\mathbf{Z}}_{10}\end{array}$ & $\begin{array}{ll}\hat{a}_{14} & 09 \\
\hat{a}_{13} & 09 \\
\hat{a}_{12} & 09 \\
\hat{a}_{11} & 09 \\
\hat{a}_{10} & 00\end{array}$ & $\begin{array}{ll}36 & (60) \\
& \end{array}$ & $\begin{array}{ll}06 & \hat{Z}_{14} \\
06 & \hat{\mathbf{Z}}_{13} \\
06 & \hat{\mathbf{Z}}_{12} \\
06 & \hat{\mathbf{Z}}_{11} \\
00 & \hat{\mathbf{Z}}_{10}\end{array}$ \\
\hline $\begin{array}{ll}\underline{\mathbf{a}}_{9} & 13 \\
\hat{a}_{8} & 14 \\
\hat{a}_{7} & 20 \\
\hat{a}_{6} & 13 \\
\underline{\hat{a}}_{5} & 16\end{array}$ & $\begin{array}{ll}07 & \hat{\mathbf{Z}}_{9} \\
08 & \hat{Z}_{8} \\
10 & \hat{\underline{Z}}_{7} \\
08 & \hat{\mathbf{Z}}_{6} \\
09 & \underline{\underline{Z}}_{5}\end{array}$ & $\begin{array}{ll}\underline{\hat{a}}_{9} & 13 \\
\hat{a}_{8} & 14 \\
\hat{\mathbf{a}}_{7} & 20 \\
\hat{\mathrm{a}}_{6} & 13 \\
\underline{\hat{a}}_{5} & 16\end{array}$ & $76(\mathbf{1 1 8}) 42$ & $\begin{array}{ll}07 & \hat{\underline{Z}}_{9} \\
08 & \hat{Z}_{8} \\
10 & \underline{\mathbf{Z}}_{7} \\
08 & \hat{\mathbf{Z}}_{6} \\
09 & \underline{\hat{Z}}_{5} \\
\end{array}$ \\
\hline $\begin{array}{ll}\hat{a}_{4} & 15 \\
\hat{a}_{3} & 13 \\
\hat{a}_{2} & 11 \\
\hat{a}_{1} & 13 \\
\hat{a}_{0} & 11\end{array}$ & $\begin{array}{ll}07 & \hat{Z}_{4} \\
05 & \hat{Z}_{3} \\
08 & \hat{Z}_{2} \\
06 & \hat{\underline{Z}}_{1} \\
09 & \hat{Z}_{0}\end{array}$ & $\begin{array}{ll}\hat{a}_{4} & 15 \\
\hat{a}_{3} & 13 \\
\hat{a}_{2} & 11 \\
\hat{a}_{1} & 13 \\
\hat{a}_{0} & 11\end{array}$ & 35 & $\begin{array}{ll}07 & \hat{Z}_{4} \\
05 & \hat{Z}_{3} \\
08 & \hat{Z}_{2} \\
06 & \hat{\underline{Z}}_{1} \\
09 & \hat{Z}_{0}\end{array}$ \\
\hline $\begin{array}{l}\text { Even } 82 \\
\text { Odd } 93\end{array}$ & 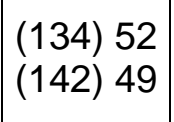 & $\begin{array}{l}145 \\
131\end{array}$ & $141 / 135$ & \\
\hline 175 & 101 & 276 & 276 & \\
\hline
\end{tabular}

Table 7.1. All branches (primary + secondary) for all the 11 species at $0-14$ levels. Notice the balances: $131 / 145$ versus $141 / 135$ as a change for \pm 10 ; then $141 / 135$ versus $142 / 134$ as a change for \pm 1 . Notice also the relations to the second (28) and the third (496) perfect number as well as the relation to the first pair of friendly numbers (220 and 284). In addition: the total number of branches (276) appears to be the first case in a specific and unique arithmetical system (Figure 4). 


\begin{tabular}{|c|c|c|c|c|}
\hline $\begin{array}{ll}\hat{\mathbf{a}}_{9} & 13 \\
\hat{\mathrm{a}}_{8} & 14 \\
\hat{\mathbf{a}}_{7} & 20 \\
\hat{\mathrm{a}}_{6} & 13 \\
\hat{\mathbf{a}}_{5} & 16\end{array}$ & $\begin{array}{ll}07 & \underline{\mathbf{Z}}_{9} \\
08 & \hat{\mathbf{Z}}_{8} \\
10 & \hat{\underline{Z}}_{7} \\
08 & \hat{\mathbf{Z}}_{6} \\
09 & \underline{\underline{\mathbf{Z}}}_{5}\end{array}$ & $\begin{array}{ll}\hat{a ̂}_{9} & 13 \\
\hat{a}_{8} & 14 \\
\hat{a}_{7} & 20 \\
\hat{a}_{6} & 13 \\
\hat{a}_{5} & 16\end{array}$ & 76 (118) 42 & 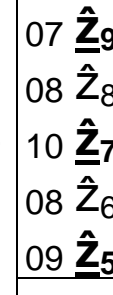 \\
\hline $\begin{array}{ll}\hat{a}_{4} & 15 \\
\hat{a}_{3} & 13 \\
\hat{a}_{2} & 11 \\
\hat{a}_{1} & 13 \\
\hat{a}_{0} & 11\end{array}$ & $\begin{array}{ll}07 & \hat{Z}_{4} \\
05 & \hat{Z}_{3} \\
08 & \hat{Z}_{2} \\
06 & \hat{Z}_{1} \\
09 & \hat{Z}_{0} \\
\end{array}$ & $\begin{array}{ll}\hat{a}_{4} & 15 \\
\hat{a}_{3} & 13 \\
\hat{a}_{2} & 11 \\
\hat{a}_{1} & 13 \\
\hat{a ̂}_{0} & 11\end{array}$ & & $\mid \begin{array}{ll}07 & \hat{\mathbf{Z}}_{4} \\
05 & \hat{\mathbf{Z}}_{3} \\
08 & \hat{\mathbf{Z}}_{2} \\
06 & \hat{\mathbf{z}}_{1} \\
09 & \hat{\mathbf{Z}}_{0}\end{array}$ \\
\hline $\begin{array}{l}\text { Odd } 75 \\
\text { Even } 64\end{array}$ & $\begin{array}{l}\text { (112) } 37 \\
\text { (104) } 40\end{array}$ & $\left(\begin{array}{l}(101) \\
(115)\end{array}\right.$ & $111 / 105$ & \\
\hline 139 & 77 & 216 & 216 & \\
\hline
\end{tabular}

Table 7.2. All branches (primary + secondary) for all the 11 species at $0-9$ levels. Notice the balances: $101 / 115$ versus $111 / 105$ as a change for \pm 10 ; then $111 / 105$ versus $112 / 104$ as a change for \pm 1 . Notice that the total number 216 is Plato's number, that is to say, the cube of number $6\left(3^{\wedge} 3+4^{\wedge} 3+5^{\wedge} 3=6^{\wedge} 3=\right.$ 216). The results $98 / 108$ appear to be in relation to a half of Plato's number, as a change for \pm 10 (108 \pm 10 ). 


\begin{tabular}{|c|c|c|c|c|}
\hline $\begin{array}{ll}\hat{a}_{14} & 09 \\
\hat{a}_{13} & 09 \\
\hat{a}_{12} & 09 \\
\hat{a}_{11} & 09 \\
\hat{a}_{10} & 00\end{array}$ & $\begin{array}{ll}06 & \hat{Z}_{14} \\
06 & \hat{\mathbf{z}}_{13} \\
06 & \hat{Z}_{12} \\
06 & \hat{\mathbf{z}}_{11} \\
00 & \hat{\mathbf{Z}}_{10}\end{array}$ & $\begin{array}{ll}\hat{a}_{14} & 09 \\
\hat{\mathbf{a}}_{13} & 09 \\
\hat{a}_{12} & 09 \\
\hat{\mathbf{a}}_{11} & 09 \\
\hat{\mathrm{a}}_{10} & 00\end{array}$ & $\begin{array}{cc}36 & (60) \\
& \end{array}$ & $\begin{array}{ll}06 & \hat{Z}_{14} \\
06 & \hat{\mathbf{Z}}_{13} \\
06 & \hat{Z}_{12} \\
06 & \hat{\mathbf{Z}}_{11} \\
00 & \hat{\mathbf{Z}}_{10}\end{array}$ \\
\hline $\begin{array}{ll}\underline{\hat{a}}_{9} & 05 \\
\hat{a}_{8} & 05 \\
\hat{a}_{7} & 07 \\
\hat{\hat{a}}_{6} & 07 \\
\underline{\hat{a}}_{5} & 06\end{array}$ & $\begin{array}{ll}02 & \hat{\underline{z}}_{9} \\
02 & \hat{Z}_{8} \\
03 & \hat{\underline{z}}_{7} \\
03 & \hat{Z}_{6} \\
03 & \underline{\underline{z}}_{5} \\
\end{array}$ & $\begin{array}{ll}\hat{a ̂}_{9} & 05 \\
\hat{a}_{8} & 05 \\
\hat{a ̂}_{7} & 07 \\
\hat{\underline{a}}_{6} & 07 \\
\hat{\mathbf{a}}_{5} & 06\end{array}$ & & $\begin{array}{ll}02 & \hat{\underline{Z}}_{9} \\
02 & \hat{Z}_{8} \\
03 & \hat{\underline{Z}}_{7} \\
03 & \hat{Z}_{6} \\
03 & \underline{\underline{Z}}_{5} \\
\end{array}$ \\
\hline $\begin{array}{ll}\hat{a}_{4} & 06 \\
\hat{a}_{3} & 06 \\
\hat{a}_{2} & 05 \\
\hat{a}_{1} & 06 \\
\hat{a}_{0} & 06\end{array}$ & $\begin{array}{ll}03 & \hat{Z}_{4} \\
02 & \hat{Z}_{3} \\
03 & \hat{Z}_{2} \\
04 & \hat{\mathbf{Z}}_{1} \\
04 & \hat{Z}_{0}\end{array}$ & $\begin{array}{ll}\hat{a}_{4} & 06 \\
\hat{a}_{3} & 06 \\
\hat{a}_{2} & 05 \\
\hat{a}_{1} & 06 \\
\hat{a}_{0} & 06 \\
\end{array}$ & $29(45)$ & $\begin{array}{ll}03 & \hat{Z}_{4} \\
02 & \hat{Z}_{3} \\
03 & \hat{Z}_{2} \\
04 & \hat{z}_{1} \\
04 & \hat{Z}_{0} \\
\end{array}$ \\
\hline $\begin{array}{ll}\text { Even } & 47 \\
\text { Odd } & 48\end{array}$ & $\begin{array}{l}\text { (74) } 27 \\
\text { (74) } 26\end{array}$ & $\begin{array}{l}75 \\
73\end{array}$ & $78 / 70$ & \\
\hline 95 & 53 & 148 & 148 & \\
\hline
\end{tabular}

Table 7.3. All primary branches for all the 11 species at $0-14$ levels. The total number 148 appears to be in relation to the half of the total number of branches (of number 276 from Table 7.1) $(148=138+10)$. Notice the balances: $78 / 70$ in this Table versus $68 / 60$ in Table 7.4 as a change for \pm 10 ; then $74 / 74$ versus $73 / 75$ as a change for \pm 1 . The result $43 / 45$ appears to be in relation to the arithmetic mean $44 / 44$ as a change for \pm 1 . 


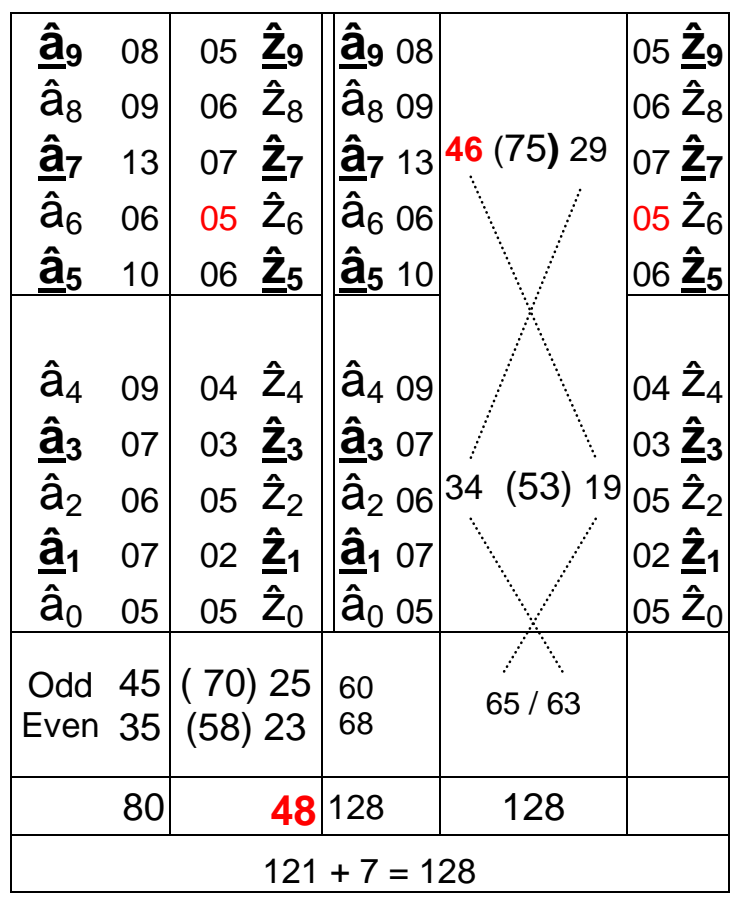

Table 7.4. All secondary branches for all the 11 species at $0-9$ levels. [The secondary branches do not exist in the upper part of the Diagram (levels 11-14)]. The total number 128 appears to be in relation to the half of the total number of branches (of number 276 from Table 7.1) $(128=138-10)$. Notice the balances: $60 / 68$ versus $70 / 58$ as a change for \pm 10 ; then $68 / 60$ in this Table versus $78 / 70$ in Table 7.3 as a change for \pm 10 ; then $74 / 74$ in Table 7.3 versus $64 \pm 1$ in this Table. 


\begin{tabular}{|c|c|c|c|c|}
\hline $\begin{array}{ll}\hat{\underline{a}}_{9} & 05 \\
\hat{\mathrm{a}}_{8} & 05 \\
\hat{\mathrm{a}}_{7} & 07 \\
\underline{\hat{\mathrm{a}}}_{6} & 07 \\
\hat{\mathrm{a}}_{5} & 06\end{array}$ & $\begin{array}{ll}02 & \hat{\mathbf{z}}_{9} \\
02 & \hat{Z}_{8} \\
03 & \hat{\underline{z}}_{7} \\
03 & \hat{Z}_{6} \\
03 & \underline{\underline{z}}_{5}\end{array}$ & $\begin{array}{ll}\hat{\underline{a}}_{9} & 05 \\
\hat{a}_{8} & 05 \\
\hat{a}_{7} & 07 \\
\underline{\hat{a}}_{6} & 07 \\
\hat{\mathbf{a}}_{5} & 06 \\
\end{array}$ & $30(\mathbf{4 3}) 13$ & $\begin{array}{ll}02 & \hat{\underline{Z}}_{9} \\
02 & \hat{Z}_{8} \\
03 & \hat{\underline{Z}}_{7} \\
03 & \hat{Z}_{6} \\
03 & \hat{\underline{Z}}_{5} \\
\end{array}$ \\
\hline $\begin{array}{ll}\hat{\mathrm{a}}_{4} & 06 \\
\hat{\mathrm{a}}_{3} & 06 \\
\hat{\mathrm{a}}_{2} & 05 \\
\hat{\mathrm{a}}_{1} & 06 \\
\hat{\mathrm{a}}_{0} & 06 \\
\end{array}$ & $\begin{array}{ll}03 & \hat{Z}_{4} \\
02 & \hat{Z}_{3} \\
03 & \hat{Z}_{2} \\
04 & \hat{z}_{1} \\
04 & \hat{Z}_{0}\end{array}$ & $\begin{array}{ll}\hat{\mathrm{a}}_{4} & 06 \\
\hat{\mathrm{a}}_{3} & 06 \\
\hat{\mathrm{A}}_{2} & 05 \\
\hat{\mathrm{a}}_{1} & 06 \\
\mathrm{a}_{1} & 06 \\
\hat{\mathrm{a}}_{0} & 06 \\
\end{array}$ & $29(\mathbf{4 5}) 16$ & $\begin{array}{ll}03 & \hat{Z}_{4} \\
02 & \hat{Z}_{3} \\
03 & \hat{Z}_{2} \\
04 & \hat{Z}_{1} \\
04 & \hat{Z}_{0}\end{array}$ \\
\hline $\begin{array}{l}\text { Even } 29 \\
\text { Odd } 30\end{array}$ & $\begin{array}{l}\text { (44) } 15 \\
\text { (44) } 14\end{array}$ & $\begin{array}{l}(45) \\
(43)\end{array}$ & $46 / 42$ & \\
\hline 59 & (88) 29 & 88 & 88 & \\
\hline \multicolumn{5}{|c|}{$56+32=88)$} \\
\hline
\end{tabular}

Table 7.5. All primary branches for all the 11 species at $0-9$ levels. The total number 88 as a result of 148 (all primary branches in Table 7.3) minus 60 branches in the upper part of the Diagram at levels 11-14 (Table 7.3). Notice the balances: $44 / 44$ versus $43 / 45$ as a change for \pm 1 ; then $43 / 45$ versus $42 / 46$ as a change for \pm 1 ; then $29 / 30$ in even/odd positions versus $29 / 30$ in up/down positions; also15/14 in even/odd positions versus $16 / 13$ in up/down positions. 


\section{Appendix A}

\begin{tabular}{|c|c|c|c|}
\hline 0 & $\begin{array}{l}11 \times 1=11 \\
11 \times 2=22 \\
11 \times 3=33\end{array}$ & $\begin{array}{l}11 \times 1=11 \\
11 \times 2=22 \\
11 \times 3=33\end{array}$ & $11^{2}=121$ \\
\hline 1 & $\begin{array}{l}12 \times 1=12 \\
12 \times 2=24 \\
12 \times 3=36\end{array}$ & $\begin{array}{l}21 \times 1=21 \\
21 \times 2=42 \\
21 \times 3=63\end{array}$ & $\begin{array}{l}12^{2}=144 \\
21^{2}=441\end{array}$ \\
\hline 2 & $\begin{array}{l}13 \times 1=13 \\
13 \times 2=26 \\
13 \times 3=39\end{array}$ & $\begin{array}{l}31 \times 1=31 \\
31 \times 2=62 \\
31 \times 3=93\end{array}$ & $\begin{array}{l}13^{2}=169 \\
31^{2}=\overline{961}\end{array}$ \\
\hline 3 & $\begin{array}{l}14 \times 1=14 \\
14 \times 2=28 \\
14 \times 3=?\end{array}$ & $\begin{array}{l}41 \times 1=41 \\
41 \times 2=82 \\
41 \times 3=?\end{array}$ & $14^{2}=196$ \\
\hline
\end{tabular}

Table A.1. The arithmetical logic square: the space of the maximum possible inversions within decimal numbering system (Rakočević, 1994, p. 235). 


\section{Appendix B}

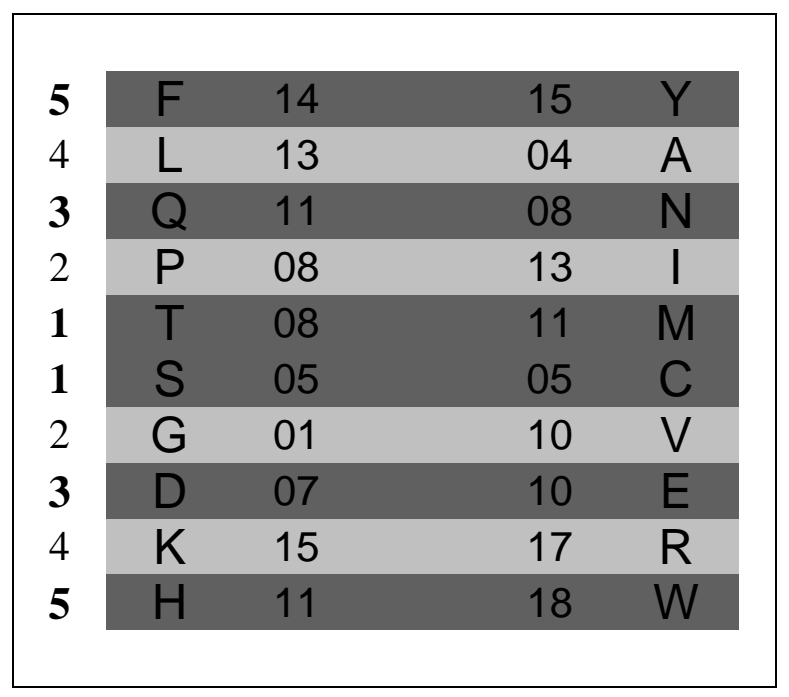

Figure B.1. "The Cyclic Invariant Periodic System (CIPS) of canonical AAs. ... In the middle position there are chalcogene AAs (S, T \& C, M); then - in the next „cycle“ - there are the AAs of non-alaninic stereochemical types $(\mathrm{G}, \mathrm{P} \& \mathrm{~V}, \mathrm{I})$, then two double acidic AAs with their two amide derivatives (D, E \& $\mathrm{N}, \mathrm{Q}$ ), the two original aliphatic AAs with two amine derivatives (A, L \& K, R); and, finally, four aromatic AAs $(F, Y \& H, W)$ - two up and two down. The said five classes belong to two super classes: primary superclass in light areas and secondary superclass in dark areas. Notice that each amino acid position in this CIPS is strictly determined and none of them can be changed" (Rakočević, 2009, Table 3; 2011, Fig. 2). 


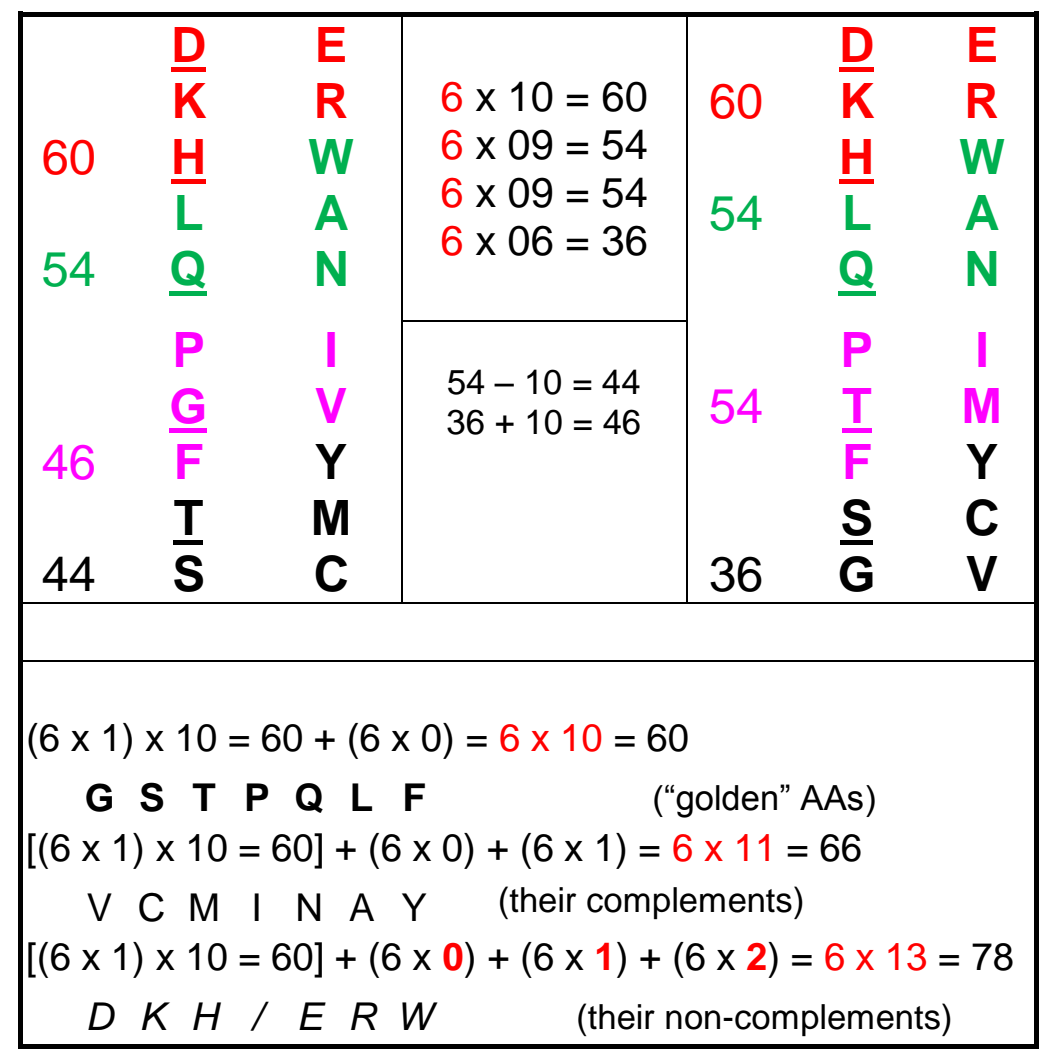

Figure B.2. This Figure follows from CIPS, presented in Figure B.1. First, there are five charged AAs. Then three other quintets follow in accordance to the three principles: principle of minimum change, principle of continuity and principle of dense packing. As it is self-evident, the system is determined by the first perfect number - the number 6. For the lower part of the Figure cf. the determination of GC by Golden mean (Rakočević, 1998a). 


\begin{tabular}{|c|c|c|c|c|c|c|}
\hline \multicolumn{6}{|c|}{ Multiples of 01, 6, 66, 666, 037 } \\
\hline 01 & 6 & 66 & 666 & $\mathbf{0 3 7}$ \\
\hline \multicolumn{6}{|c|}{$162=216-(2 \times 27)$} \\
\hline 27 & $\mathbf{1 6 2}$ & 1782 & 162 & $\mathbf{9 9 9}$ \\
26 & 156 & 1716 & 17316 & $\mathbf{9 6 2}$ \\
25 & 150 & 1650 & 16650 & $\mathbf{9 2 5}$ \\
$\ldots .$. & & & & \\
13 & 78 & 858 & $\mathbf{8 6 5 8}$ & $\mathbf{4 8 1}$ \\
12 & 72 & 792 & 7992 & $\mathbf{4 4 4}$ \\
11 & 66 & 726 & 7326 & $\mathbf{4 0 7}$ \\
$\ldots .$. & & & & \\
03 & 18 & 198 & 1998 & $\mathbf{1 1 1}$ \\
02 & 12 & 132 & 1332 & $\mathbf{0 7 4}$ \\
01 & 6 & 66 & 666 & $\mathbf{0 3 7}$ \\
\hline \multicolumn{7}{|c|}{ The 216 as Plato’s number (6^3=216) } \\
\hline
\end{tabular}

Table B.1. The multiples of the numbers are presented in the first row. The $13^{\text {th }}$ case is the sum of the first four perfect numbers $(6+28+496+8128=8658)$.

\begin{tabular}{|l|l|}
\hline $6=1 / 3=(0.333 \ldots) \times 18$ & $6 \times 11=\mathbf{6 6}(60+06)$ \\
$66=11 / 3=0.666 \ldots) \times 18$ & $66 \times 11=726(660+066)$ \\
$666=111 / 3=\quad \mathbf{0 3 7} \times 18$ & $666 \times 11=7326(6660+0666)$ \\
& $111+66=177$ \\
\hline$(1 \times 037)+(2 \times 037)=111$ & \\
\hline
\end{tabular}

Survey B.1. This first "mirror" corresponding case (66) and the first integer case (037) correspond to Darwin's diagram through the results in Table 4.3. 


\begin{tabular}{|l|c|c|c|c|c|c|}
\hline \multicolumn{5}{|c|}{ Multiples of 01, 7, 77, 777, 037} \\
\hline 01 & 7 & 77 & 777 & $\mathbf{0 3 7}$ \\
\hline \multicolumn{6}{|c|}{$189=216-(1 \times 27)$} \\
\hline 27 & $\mathbf{1 8 9}$ & 2079 & 20979 & $\mathbf{9 9 9}$ \\
26 & 182 & 2002 & 20202 & $\mathbf{9 6 2}$ \\
25 & 175 & 1925 & 19425 & $\mathbf{9 2 5}$ \\
$\ldots$. & & & & \\
13 & 91 & 1001 & $\mathbf{1 0 1 0 1}$ & $\mathbf{4 8 1}$ \\
12 & 84 & 924 & 9324 & $\mathbf{4 4 4}$ \\
11 & 77 & 847 & 8547 & $\mathbf{4 0 7}$ \\
$\ldots$. & & & & \\
03 & 21 & 231 & 2331 & $\mathbf{1 1 1}$ \\
02 & 14 & 154 & 1554 & $\mathbf{0 7 4}$ \\
01 & 7 & 77 & 777 & $\mathbf{0 3 7}$ \\
\hline \multicolumn{7}{|c|}{ The 216 as Plato's number $\left(6^{\wedge} 3=216\right)$} \\
\hline
\end{tabular}

Table B.2. The multiples of the numbers presented in the first row. The $13^{\text {th }}$ case corresponds to the line of maximal changes (the change in each following step) on the binary tree (Rakočević, 1998).

\begin{tabular}{|l|l|}
\hline $\begin{array}{l}7=1 / 3=(0.333 \ldots) \times 21 \\
77=11 / 3=0.666 \ldots) \times 21 \\
777=111 / 3=\quad \mathbf{0 3 7} \times 21\end{array}$ & $\begin{array}{l}7 \times 11=\mathbf{7 7}(70+07) \\
777 \times 11=847(770+077)\end{array}$ \\
\hline$(1 \times 037)+(2 \times 037)=111$ & $111+77=188$ \\
\hline
\end{tabular}

Survey B.2. This first "mirror" corresponding case (77) and the first integer case (037) correspond to Darwin's diagram through the results in Survey B.4 (middle area with dark tones). 


\begin{tabular}{|c|c|c|c|c|c|c|}
\hline \multicolumn{6}{|c|}{ Multiples of 01, 8, 88, 888, 037 } \\
\hline 01 & 8 & \multicolumn{2}{|c|}{88} & 888 & $\mathbf{0 3 7}$ \\
\hline \multicolumn{6}{|c|}{$216=216 \pm(0 \times 27)$} \\
\hline 27 & $\mathbf{2 1 6}$ & 2376 & 23976 & $\mathbf{9 9 9}$ \\
26 & 208 & 2288 & 23088 & $\mathbf{9 6 2}$ \\
25 & 200 & 2200 & 22200 & $\mathbf{9 2 5}$ \\
$\ldots$ & & & & \\
13 & 104 & 1144 & $\mathbf{1 1 5 4 4}$ & $\mathbf{4 8 1}$ \\
12 & 96 & 1056 & 10656 & $\mathbf{4 4 4}$ \\
11 & 88 & 968 & 9768 & $\mathbf{4 0 7}$ \\
$\ldots$ & & & & \\
03 & 24 & 264 & 2664 & $\mathbf{1 1 1}$ \\
02 & 16 & 176 & 1776 & $\mathbf{0 7 4}$ \\
01 & 8 & 88 & 888 & $\mathbf{0 3 7}$ \\
\hline \multicolumn{6}{|c|}{$\left(3^{\wedge} 3=27\right)\left(6^{\wedge} 3=216\right)$} \\
\hline
\end{tabular}

Table B.3. The multiples of the numbers are presented in the first row. The Plato's number 216 (the cube of number 6) appears as the last result in column of number " 8 ".

\begin{tabular}{|l|l|}
\hline $8=1 / 3=(0.333 \ldots) \times 24$ & $8 \times 11=\mathbf{8 8}(80+08)$ \\
$88=11 / 3=0.666 \ldots) \times 24$ & $88 \times 11=968(880+088)$ \\
$888=111 / 3=\quad \mathbf{0 3 7} \times 24$ & $888 \times 11=9768(8880+0888)$ \\
\hline$(1 \times 037)+(2 \times 037)=111$ & $111+88=199$ \\
\hline
\end{tabular}

Survey B.3. This first "mirror" corresponding case (88) and the first integer case (037) correspond to Darwin's diagram through the results in Survey B.4 (middle area in dark tones). 


\begin{tabular}{|c|c|c|c|}
\hline \multicolumn{2}{|c|}{$\begin{array}{l}(1 \times 037)+(2 \times 037)=111 \\
\mathbf{2 7} \times 037=999\end{array}$} & $\begin{array}{l}111+66=177 \\
177-56=121\end{array}$ & $\begin{array}{l}177-65=112 \\
121+112=233\end{array}$ \\
\hline $\begin{array}{l}(30 / 37 / 77) \\
(30 / 27 / 67)\end{array}$ & $\begin{array}{l}6^{\wedge} 1=6 \\
5^{\wedge} 2=25(31)\end{array}$ & $\begin{array}{l}177+077=254 \\
177=50+127\end{array}$ & $\begin{array}{l}254=117+137 \\
254=50+204\end{array}$ \\
\hline \multicolumn{2}{|c|}{$\begin{array}{l}(1 \times 037)+(2 \times 037)=111 \\
27 \times 037=999\end{array}$} & $\begin{array}{l}111+77=188 \\
188-67=121\end{array}$ & $\begin{array}{l}188-76=112 \\
121+112=233\end{array}$ \\
\hline $\begin{array}{l}(30 / 37 / 77) \\
(30 / 27 / 67)\end{array}$ & $\begin{array}{l}7^{\wedge} 1=7 \\
6^{\wedge} 2=36(43)\end{array}$ & $\begin{array}{l}188+088=276 \\
188=60+128\end{array}$ & $\begin{array}{l}276=128+148 \\
\mathbf{2 7 6}=\mathbf{6 0}+\mathbf{2 1 6}\end{array}$ \\
\hline \multicolumn{2}{|c|}{$\begin{array}{l}(1 \times 037)+(2 \times 037)=111 \\
\mathbf{2 7} \times \mathbf{0 3 7}=\mathbf{9 9 9}\end{array}$} & $\begin{array}{l}111+88=199 \\
199-78=121\end{array}$ & $\begin{array}{l}199-87=112 \\
121+112=233\end{array}$ \\
\hline $\begin{array}{l}(30 / 37 / 77) \\
(30 / 27 / 67) \\
\end{array}$ & $\begin{array}{l}8^{\wedge} 1=8 \\
7^{\wedge} 2=49(57)\end{array}$ & $\begin{array}{l}199+099=298 \\
199=70+129\end{array}$ & $\begin{array}{l}298=139+159 \\
298=70+228\end{array}$ \\
\hline
\end{tabular}

Survey B.4. The first area corresponds to Table B.1 and Survey B.1; the second (in dark tones) to Table B.2 and Survey B.2; and the third area corresponds to Table B.3 and Survey B.3. The middle area is especially significant because it, mutatis mutandis, contains all Darwin's quantities in relation to Mendeleev's quantitatives (the same area, on the left: 30/37/77 versus 30/27/67) (cf. Section 4.7, last paragraph, and Mendeleev's manuscript photocopy - Photocopy X in Kedrov, 1977, pp. 128-129). 


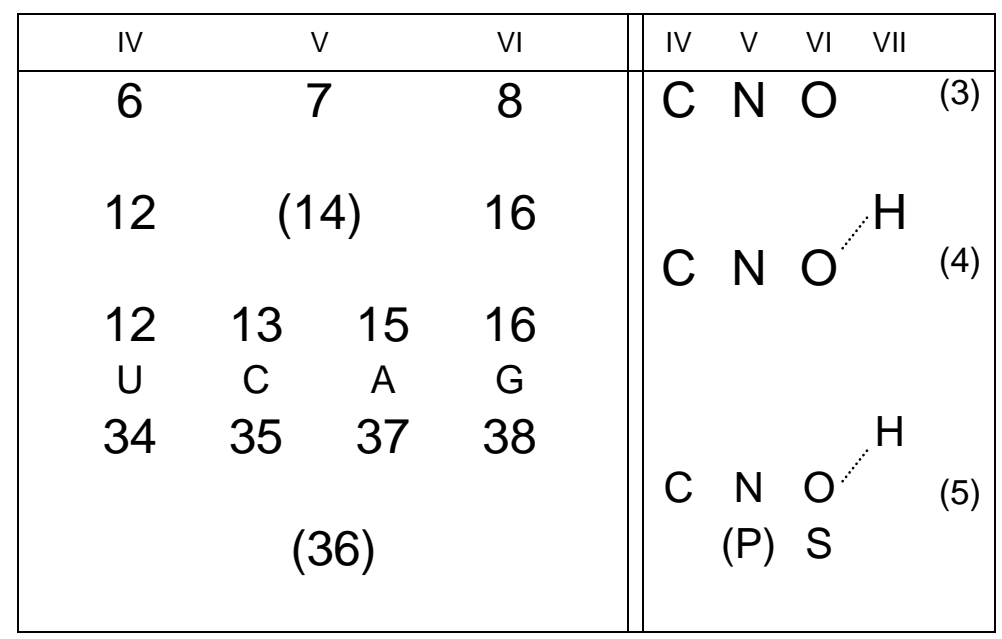

Survey B.5. A hypothetical model for the connection between the quantities/entities in Tables B.1, B.2 and B.3 and 6-7-8 proton determined chemical elements (C-N-O) as constituents of life anywhere in the universe. On the left: 6, 7, 8 protons for first three elements in IV-V-VI group of Periodic system of chemical elements, respectively; then 12, 14, 16 nucleons of these elements; then 12, 13, 15, 16 atoms in four $\mathrm{Py} / \mathrm{Pu}$ bases, with the relation to the half of second perfect number (28); in the last row, there is the number of atoms within four nucleotide molecules in relation to the cube of the first perfect number, number 6. [Notice that the number of nucleons in the second row and the number of atoms in the third row represent a unique type of self-similarity.] On the right: 3, 4 and 5 chemical elements as constituents of protein amino acids - the constituents of proteins. Notice that the last case on the right represents five elements in amino acid molecules $(\mathrm{C}, \mathrm{N}, \mathrm{O}, \mathrm{S}, \mathrm{H})$ and five elements in nucleotide molecules $(\mathrm{C}, \mathrm{N}, \mathrm{O}, \mathrm{P}, \mathrm{H})$ at the same time. Notice also that hydrogen, as a nonmetal, exists within the seventh group of Periodic system. Altogether it is self-evident that the neighbor positions of life-elements are determined with the three principles: principle of minimum change, principle of continuity and the principle of neighborhood. 


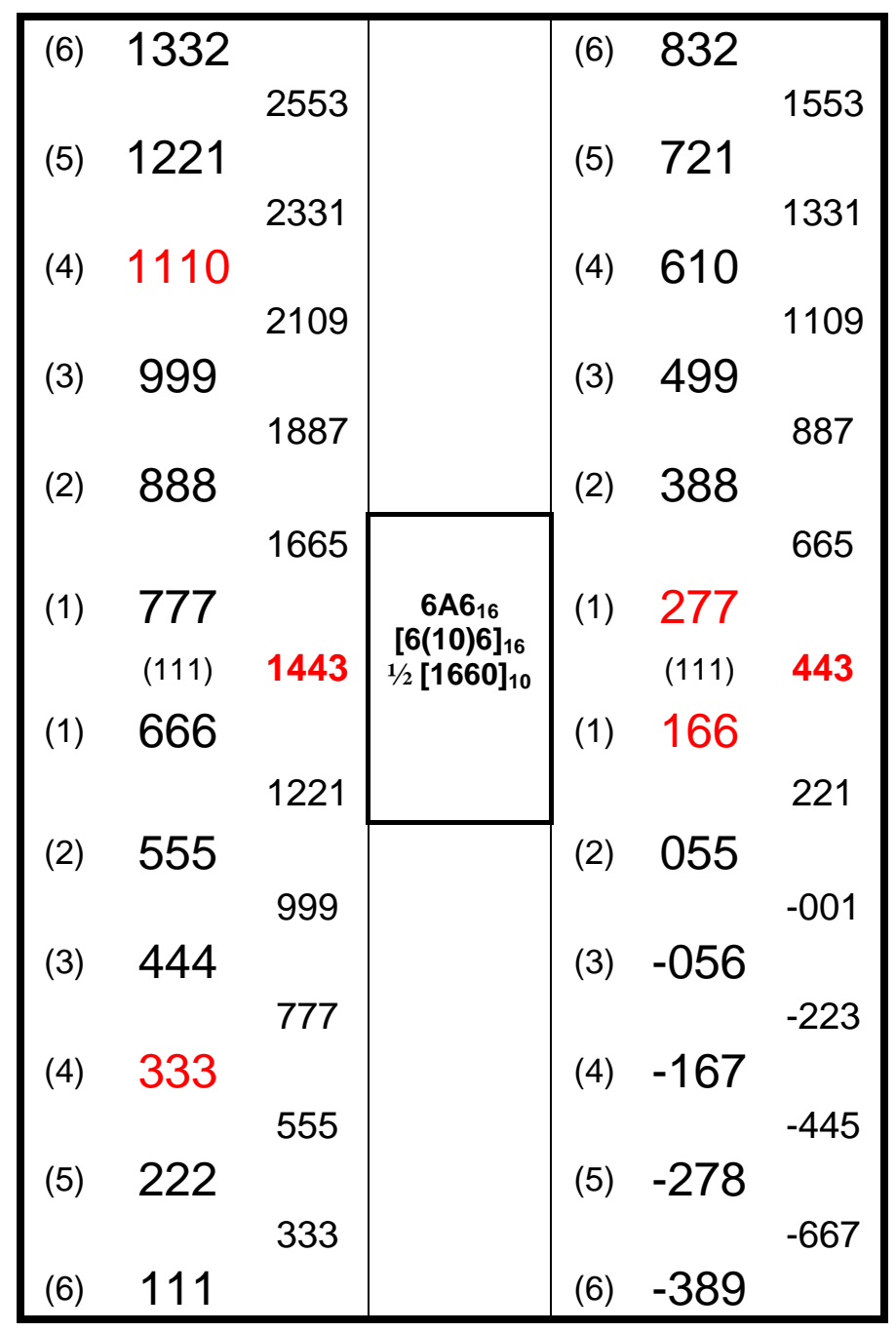

Survey B.6. If multiples 666 (Table B.1) and 777 (Table B.2) have a middle position within the system of presented multiples, then it becomes obvious that there are the relations to the number of nucleons as well as of atoms within amino acid molecules as constituents of the Genetic code. Number 1443 as the number of nucleons within 23 amino acid molecules, within their side chains, in Shcherbak's diagram (Figure 3). Notice that number 1443 is $1 / 6$ of the sum of the first four perfect numbers $(6+28+496+8128=8658=6 \times 1443)$ and the sum of all multiples in the second column of this Table at the same time. Within 23 amino acid "heads" (amino acid functional groups) there are 1702 nucleons written in decimal numbering system, or 6(10)6 (i.e. 6A6) in hexadecimal system (see the window in the middle frame area). Number 443 as the number of atoms within 43 amino acid molecules (within their side chains) after the arrangement in Table E.1 (row "d"). Within 43 amino acid "heads" there are 387 atoms. The sum $443+387$ equals $1 / 2$ of 1660 written in decimal numbering system. [Notice the two designations: 6(10)6 for nucleon number and 1660 for atom number express a specific self-similarity.] 


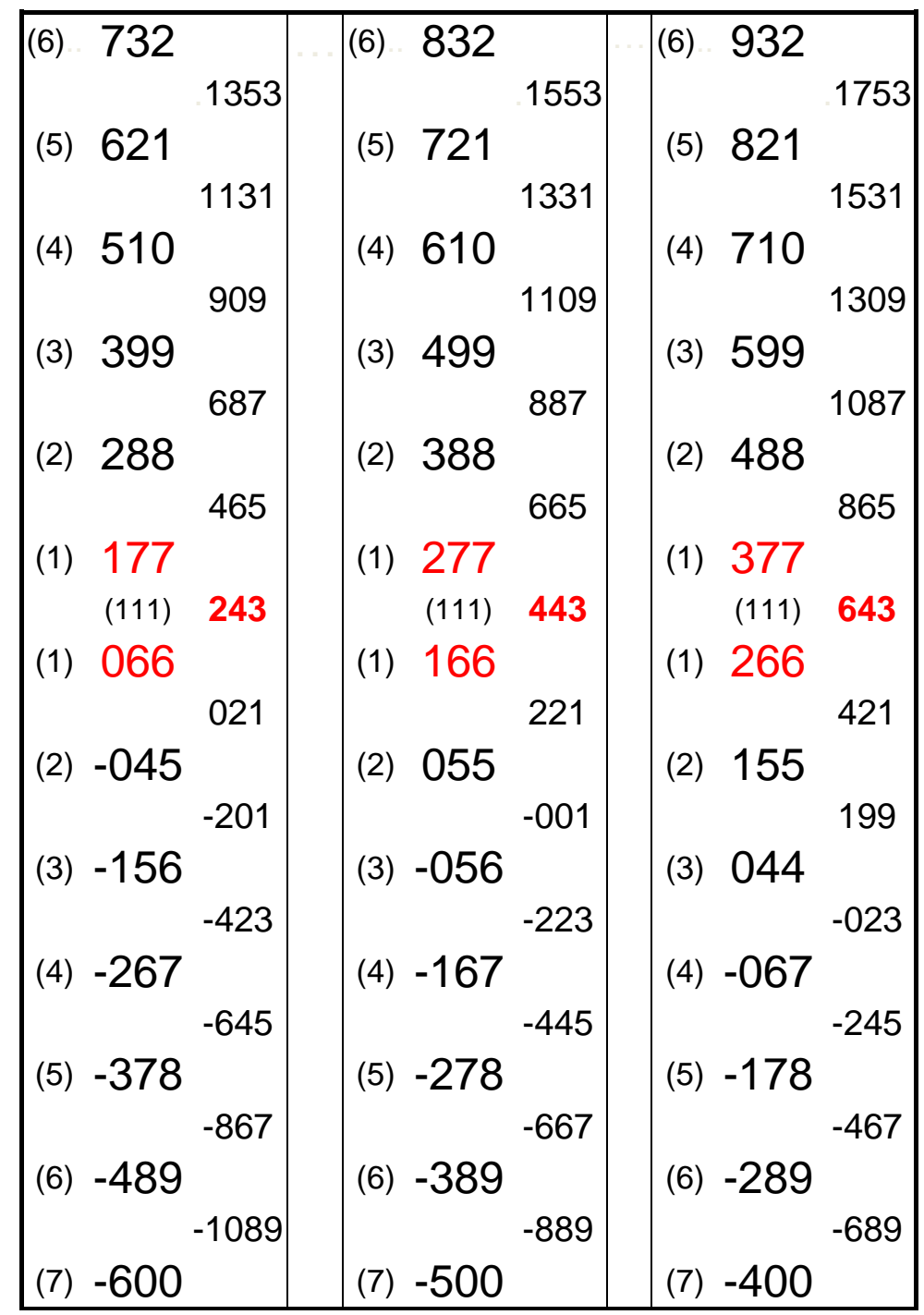

Survey B.7. The arithmetical system which is in relation with the system, presented in Survey B.6. (Notice that the last difference in "Darwin's column" is $1089=33^{\wedge} 2$; in "Genetic code's" column $1089-200$, and in the third, the "neutral" column it is $1089-400$.) 


\begin{tabular}{|c|c|c|c|c|c|c|c|}
\hline & & $\mathrm{N}$ & $\mathrm{a}_{1}, \mathrm{a}_{2}$ & $\mathrm{~A}$ & $\mathrm{D}$ & $\mathrm{d}_{1}$ & $\mathrm{~d}_{2}$ \\
\hline$(4)$ & 999 & 1887 & 499 & & 787 & 7111 & 111 \\
$(3)$ & 888 & 1665 & 388 & & & & \\
\hline$(2)$ & $\mathbf{7 7 7}$ & 1443 & $\mathbf{2 7 7}$ & $\mathbf{4 4 3}$ & $\mathbf{3 3 2}$ & $\mathbf{1 1 1 1}$ & $\mathbf{1 1 1}$ \\
$(\mathbf{1})$ & $\mathbf{6 6 6}$ & $\mathbf{1 4 4 6}$ & $\mathbf{1 6 6}$ & & & & \\
\hline$(0)$ & 555 & 1221 & 055 & 221 & 110 & 1111 & 111 \\
\hline$(-1)$ & 444 & 999 & & & & & \\
$(-2)$ & 333 & 777 & & & & & \\
$(-3)$ & 222 & 555 & & & & & \\
$(-4)$ & 111 & 333 & & & & & \\
\hline
\end{tabular}

Survey B.8. An insert from Survey B.6; N: the numbers in relation to nucleon number 1443; $a_{1}$, $\mathrm{a}_{2}$ : the numbers in relation to atom number 166 and 277, respectively; A: the numbers in relation to atom number 443; D: the numbers in relation to Darwin's number 332 as the total number of "branch" quantities/entities in his Diagram (Table 5); $\mathrm{d}_{1}$ : all differences in relation to the difference $1443-332=1111 ; \mathrm{d}_{2}$ : all differences in relation to the difference $443-332=111$. 


\section{Appendix C}

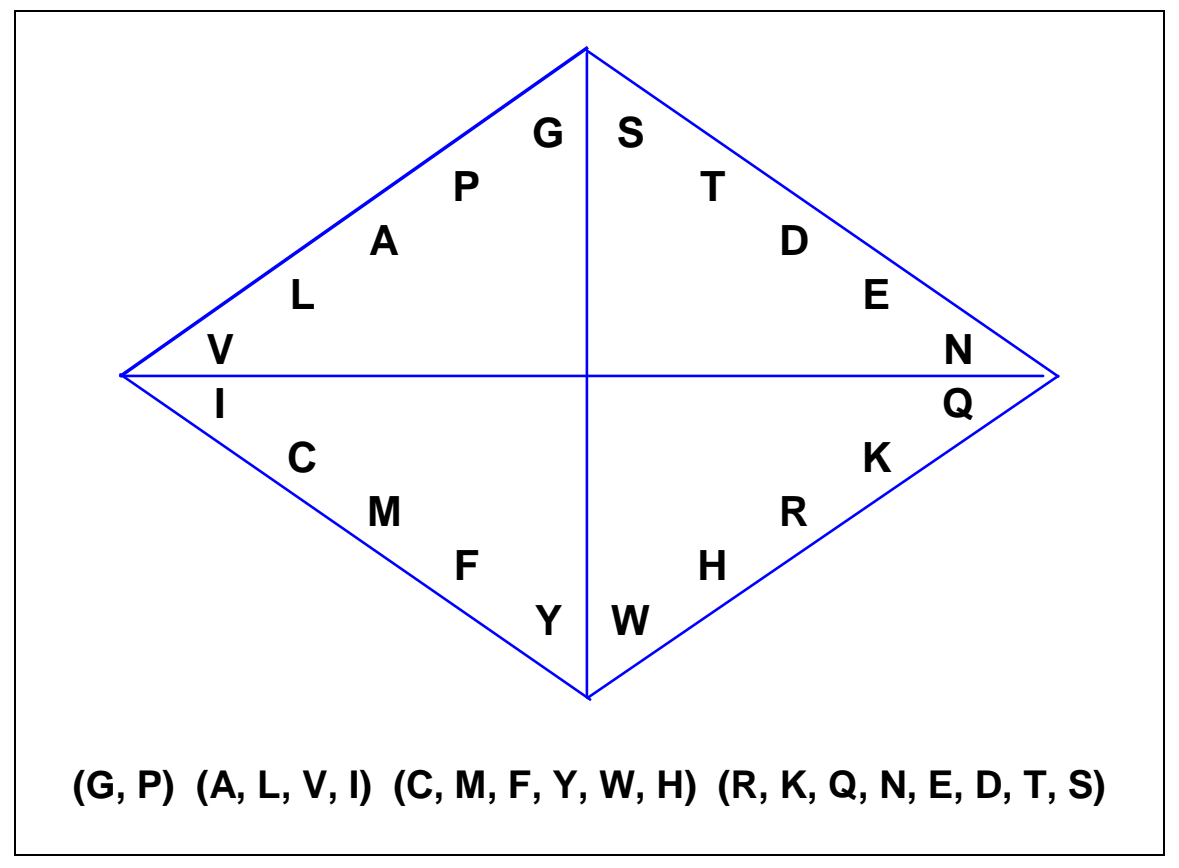

Figure C.1. Four diversity types of protein amino acids: 2 AAs with non-standard and 4 AAs with standard hydrocarbon side chain; then 6 AAs with different, and 8 with the same "head"/"body" functional groups: linear and circular arrangement, which from - through the principles of minimum change and continuity - follows a new arrangement, such as in Figure C.2 (Rakočević, 2011a, Fig. 2; 2011b, Fig. 2 on p. 822). 


\begin{tabular}{|c|c|c|c|c|c|c|}
\hline G 01 & S 05 & Y 15 & W 18 & 39 & \multirow{2}{*}{78} & \multirow[b]{2}{*}{102} \\
\hline A 04 & D 07 & $\mathrm{M} 11$ & R 17 & 39 & & \\
\hline C 05 & Т 08 & E 10 & $F_{14}$ & 37 & 24 & \\
\hline N 08 & Q 11 & V 10 & I 13 & 42 & 89 & 102 \\
\hline $\mathrm{P} 08$ & $\mathrm{H} 11$ & L 13 & K 15 & 47 & & \\
\hline 26 & 42 & 59 & 77 & & & \\
\hline & $\begin{array}{c}16 \\
1 \times 68)\end{array}$ & $\begin{array}{l}17 \\
(2\end{array}$ & & & & \\
\hline
\end{tabular}

Figure C.2. A specific AA classification and systematization which follow from four diversity types (Figure C.1) in correspondence with a unique arithmetical arrangement (Table C.2). The ordering through the validity of two Mendeleev principles: minimum change and continuity $(1,5,15,18$ of atoms in the first row), (1, 4, 5, 8 of atoms in the first column) (Rakočević, 2011a, Fig. 1; 2011b, Fig. 3 on p. 828).

\begin{tabular}{|c|c|c|c|c|c|c|c|c|c|c|c|}
\hline$(-2)$ & & & & & & & & & & & -22 \\
\hline$(-1)$ & -21 & -20 & -19 & -18 & -17 & -16 & -15 & -14 & -13 & -12 & -11 \\
\hline (0) & -10 & -09 & -08 & -07 & -06 & -05 & -04 & -03 & -02 & -01 & 00 \\
\hline (1) & 01 & 02 & 03 & 04 & 05 & 06 & 07 & 08 & 09 & 10 & 11 \\
\hline (2) & 12 & 13 & 14 & 15 & $16^{-}$ & $-17-$ & -18 & 19 & 20 & 21 & 22 \\
\hline (3) & 23 & 24 & 25 & $26^{\prime}$ & 27 & 28 & 29 & 30 & 31 & 32 & 33 \\
\hline (4) & 34 & 35 & 36 & 37 & 38 & 39 & 40 & 41 & 42 & 43 & 44 \\
\hline (5) & 45 & 46 & 47 & 48 & 49 & 50 & 51 & 52 & 53 & 54 & 55 \\
\hline (6) & 56 & 57 & 58 & 59 & 60 & $5 B$ & 62 & 63 & 64 & 65 & 66 \\
\hline (7) & 67 & 68 & 69 & 70 & 71 & 72 & 6D & 74 & 75 & 76 & 77 \\
\hline (8) & 78 & 79 & 80 & 81 & 82 & 83 & 84 & $7 F$ & 86 & 87 & 88 \\
\hline (9) & 89 & 90 & 91 & 92 & 93 & 94 & 95 & 96 & 97 & 98 & 99 \\
\hline (A) & A0 & $\mathrm{A} 1$ & A2 & A3 & A4 & A5 & A6 & A7 & A8 & A9 & $A A$ \\
\hline (B) & B1 & B2 & B3 & B4 & B5 & B6 & B7 & B8 & B9 & BA & BB \\
\hline
\end{tabular}

Table C.1. The Table of minimal addition in decimal numbering system. A specific arrangement of natural numbers in decimal numbering system, going from 01 to 11 and so on (Rakočević, 2011a, Tab. 4; 2011b, Tab. 4 on p. 826). 


\begin{tabular}{|lll|}
\hline $26 \quad=26$ & $26+42+59+77=\mathrm{Y}$ & $\mathbf{1 6}+\mathbf{1 7}+\mathbf{1 8}=\mathrm{Z}$ \\
$26+\mathbf{1 6}=42$ & $\mathrm{Y}=204$ & $\mathrm{Z}=51$ \\
$42+\mathbf{1 7}=59$ & $\mathrm{Y} / 4=51$ & $\mathrm{Z}=\mathrm{Y} / 4$ \\
$59+\mathbf{1 8}=77$ & & \\
\hline
\end{tabular}

Survey C.1. The unique arithmetical relations which follow from the system presented in Table C.1 (Rakočević, 2011a, Equations 4.1; 2011b, Equations 3 on p. 826).

\begin{tabular}{|ll|}
$\mathrm{x}_{1}+\mathrm{y}_{1}=36=\mathbf{6}^{2}$ & $\left(\mathrm{x}_{1}=26 ; \mathrm{y}_{1}=10\right)$ \\
$\mathrm{x}_{2}+\mathrm{y}_{2}=25=\mathbf{5}^{\mathbf{2}}$ & $\left(\mathrm{x}_{2}=17 ; \mathrm{y}_{2}=08\right)$ \\
$\mathrm{x}_{1}-\mathrm{y}_{1}=16=\mathbf{4}^{\mathbf{2}}$ & \\
$\mathrm{x}_{2}-\mathrm{y}_{2}=09=\mathbf{3}^{\mathbf{2}}$ & \\
& \\
\hline
\end{tabular}

Survey C.2. The unique algebraic relations which follow from the system presented in Table C.1 (Rakočević, 2011a, Equations 4.2; 2011b, Equations 4 on p. 827). 


\begin{tabular}{|c|c|c|c|c|c|c|c|c|c|c|c|}
\hline$(-2)$ & $\begin{array}{l}\cdots \\
\ldots\end{array}$ & & & & & & & & & & -22 \\
\hline$(-1)$ & -21 & -20 & -19 & -18 & -17 & -16 & -15 & -14 & -13 & -12 & -11 \\
\hline (0) & -10 & -09 & -08 & -07 & -06 & -05 & -04 & -03 & -02 & -01 & 00 \\
\hline (1) & $\underline{0} 1$ & 02 & 03 & 04 & 05 & 06 & 07 & 08 & 09 & 10 & 11 \\
\hline (2) & 12 & 13 & 14 & 15 & $16^{-}$ & $-17-$ & -18 & 19 & 20 & 21 & 22 \\
\hline (3) & $\underline{2} 3$ & 24 & 25 & $26^{\prime}$ & 27 & 28 & 29 & 30 & 31 & 32 & 33 \\
\hline (4) & $\underline{3} 4$ & 35 & 36 & 37 & 38 & 39 & 40 & 41 & 42 & 43 & 44 \\
\hline (5) & 45 & 46 & 47 & 48 & 49 & 50 & 51 & 52 & 53 & 54 & 55 \\
\hline (6) & $\underline{5} 6$ & 57 & 58 & 59 & 60 & 61 & 62 & 63 & 64 & 65 & 66 \\
\hline (7) & $\underline{6} 7$ & 68 & 69 & 70 & 71 & 72 & 73 & 74 & 75 & 76 & 77 \\
\hline (8) & 78 & 79 & 80 & 81 & 82 & 83 & 84 & 85 & 86 & 87 & 88 \\
\hline (9) & 89 & 90 & 91 & 92 & 93 & 94 & 95 & 96 & 97 & 98 & 99 \\
\hline (10) & 100 & 101 & 102 & 103 & 104 & 105 & 106 & 107 & 108 & 109 & 110 \\
\hline 1) & 111 & 112 & 113 & 114 & 115 & 116 & 117 & 118 & 119 & 120 & 121 \\
\hline
\end{tabular}

Table C.2. This Table is the same as Table C.1, except the first, highlighted column and the left diagonal, so that the following law is to be detected: the left diagonal appears as the sum of all neighboring pairs in the first column minus $10 \mathrm{n}$, where $\mathrm{n}=0,1,2, \ldots$ 


\begin{tabular}{|c|c|c|c|c|c|c|c|c|c|ll|}
\hline $01(-10)$ & -9 & -8 & -7 & -6 & -5 & -4 & -3 & -2 & -1 & 0 & \\
\hline & 21 & 32 & 43 & 54 & 65 & 76 & 87 & 98 & 109 & 120 & \\
\hline 12 & 012 & 024 & 036 & 048 & 060 & 072 & 084 & 096 & 108 & 120 & $(220)$ \\
\hline & 241 & 252 & 263 & 274 & 285 & 296 & 307 & 318 & 329 & 340 & \\
\hline 23 & 253 & 276 & 299 & 322 & 345 & 368 & 391 & 414 & 437 & 460 & $(220)$ \\
\hline & 461 & 472 & 483 & 494 & 505 & 516 & 527 & 538 & 549 & 560 & \\
\hline 34 & 714 & 748 & 782 & 816 & 850 & 884 & 918 & 952 & 986 & 1020 & $(220)$ \\
\hline & 681 & 692 & 703 & 714 & 725 & 736 & 747 & 758 & 769 & 780 & \\
\hline 45 & 1395 & 1440 & 1485 & 1530 & 1575 & 1620 & 1665 & 1710 & 1755 & 1800 & $(220)$ \\
\hline & 901 & 912 & 923 & 934 & 945 & 956 & 967 & 978 & 989 & 1000 & \\
\hline 56 & 2296 & 2352 & 2408 & 2464 & 2520 & 2576 & 2632 & 2688 & 2744 & 2800 & $(220)$ \\
\hline & 1121 & 1132 & 1143 & 1154 & 1165 & 1176 & 1187 & 1198 & 1209 & 1220 & \\
\hline 67 & 3417 & 3484 & 3551 & 3618 & 3685 & 3752 & 3819 & 3886 & 3953 & 4020 & $(220)$ \\
\hline & 1341 & 1352 & 1363 & 1374 & 1385 & 1396 & 1407 & 1418 & 1429 & 1440 & \\
\hline 78 & 4758 & 4836 & 4914 & 4992 & 5070 & 5148 & 5226 & 5304 & 5382 & 5460 & $(220)$ \\
\hline & 1561 & 1572 & 1583 & 1594 & 1605 & 1616 & 1627 & 1638 & 1649 & 1660 & \\
\hline 89 & 6319 & 6408 & 6497 & 6586 & 6675 & 6764 & 6853 & 6942 & 7031 & 7120 \\
\hline
\end{tabular}

Table C.3. The Table follows from Table C.2 with the multiplication of all neighbouring pairs in first column (the numbers on the diagonal), of their predecessors (the numbers for the diagonal) and of their successors (the numbers after the diagonal). The differences increase by 11 , and the differences of differences by the twentieth multiple of 11 , the number 220 , which is the first friendly number. Here one must notice that the numbers on the left diagonal are the same numbers which appear in the arithmetical system presented in Figure 4. 


\begin{tabular}{|c|c|c|c|c|c|c|c|c|c|}
\hline \multirow{2}{*}{$\begin{array}{l}1 \mathrm{st} \\
\text { lett. }\end{array}$} & \multicolumn{8}{|c|}{ 2nd letter } & \multirow{2}{*}{$\begin{array}{l}\text { 3rd } \\
\text { lett. }\end{array}$} \\
\hline & U & & C & & $A$ & & $\mathrm{G}$ & & \\
\hline U & $\begin{array}{l}\text { 00. UUU } \\
\text { 01. UUC } \\
\text { 02. UUA } \\
\text { 03. UUG }\end{array}$ & $\mathbf{L}$ & $\begin{array}{l}\text { 08. UCU } \\
\text { 09. UCC } \\
\text { 10. UCA } \\
\text { 11. UCG }\end{array}$ & $S$ & $\begin{array}{l}\text { 32. UAU } \\
\text { 33. UAC } \\
\text { 34. UAA } \\
\text { 35. UAG }\end{array}$ & $\begin{array}{l}\mathbf{Y} \\
\mathrm{CT}\end{array}$ & $\begin{array}{l}\text { 40. UGU } \\
\text { 41. UGC } \\
\text { 42. UGA } \\
\text { 43. UGG }\end{array}$ & $\begin{array}{l}\mathbf{C} \\
\mathrm{CT} \\
\mathbf{W}\end{array}$ & $\begin{array}{l}\mathrm{U} \\
\mathrm{C} \\
\mathrm{A} \\
\mathrm{G}\end{array}$ \\
\hline C & $\begin{array}{l}\text { 04. CUU } \\
\text { 05. CUC } \\
\text { 06. CUA } \\
\text { 07. CUG }\end{array}$ & $\mathbf{L}$ & $\begin{array}{l}\text { 12. CCU } \\
\text { 13. CCC } \\
\text { 14. } \mathrm{CCA} \\
\text { 15. CCG }\end{array}$ & $\mathbf{P}$ & $\begin{array}{l}\text { 36. CAU } \\
\text { 37. CAC } \\
\text { 38. CAA } \\
\text { 39. CAG }\end{array}$ & $\begin{array}{l}\mathbf{H} \\
\mathbf{Q}\end{array}$ & $\begin{array}{l}\text { 44. CGU } \\
\text { 45. } \mathrm{CGC} \\
\text { 46. CGA } \\
\text { 47. CGG }\end{array}$ & $\mathbf{R}$ & $\begin{array}{l}\text { U } \\
\text { C } \\
\text { A } \\
\text { G }\end{array}$ \\
\hline A & $\begin{array}{l}\text { 16. AUU } \\
\text { 17. AUC } \\
\text { 18. AUA } \\
\text { 19. AUG }\end{array}$ & I & $\begin{array}{l}\text { 24. ACU } \\
\text { 25. ACC } \\
\text { 26. ACA } \\
\text { 27. ACG }\end{array}$ & $\mathbf{T}$ & $\begin{array}{l}\text { 48. AAU } \\
\text { 49. AAC } \\
\text { 50. AAA } \\
\text { 51. AAG }\end{array}$ & $\begin{array}{l}\mathbf{N} \\
\mathbf{K}\end{array}$ & $\begin{array}{l}\text { 56. AGU } \\
\text { 57. AGC } \\
\text { 58. AGA } \\
\text { 59. AGG }\end{array}$ & $\mathbf{R}$ & $\begin{array}{l}\text { U } \\
\text { C } \\
\text { A } \\
\text { G }\end{array}$ \\
\hline $\mathrm{G}$ & $\begin{array}{l}\text { 20. GUU } \\
\text { 21. GUC } \\
\text { 22. GUA } \\
\text { 23. GUG }\end{array}$ & V & $\begin{array}{l}\text { 28. GCU } \\
\text { 29. GCC } \\
\text { 30. GCA } \\
\text { 31. GCG }\end{array}$ & $\mathbf{A}$ & $\begin{array}{l}\text { 52. GAU } \\
\text { 53. GAC } \\
\text { 54. GAA } \\
\text { 55. GAG }\end{array}$ & $\mathbf{E}$ & $\begin{array}{l}\text { 60. GGU } \\
\text { 61. GGC } \\
\text { 62. GGA } \\
\text { 63. GGG }\end{array}$ & $\mathbf{G}$ & $\begin{array}{l}\text { U } \\
\text { C } \\
\text { A } \\
\text { G }\end{array}$ \\
\hline
\end{tabular}

Table C.4. The standard Genetic Code Table. This Table represents the relations within the so cold "standard Genetic code" with designation of four diversity types of protein amino acids and corresponding codons: the first and the second type without color (in light and dark tones, respectively), but the third and the fourth in color. The codon number: for the first type 08, the second 17, the third 10 and the fourth 26, just as in algebraic system in Survey C.2 (Rakočević, 2011a, Fig. 3; 2011b, Tab. 6 on p. 829). 


\section{Appendix D}

\begin{tabular}{|c|c|c|c|c|c|c|c|c|c|}
\hline Fibonacci & 0 & 1 & 1 & 2 & 3 & 5 & 8 & 13 & $\ldots$ \\
\hline Fibonacci & 1 & 2 & 3 & 5 & 8 & 13 & 21 & 34 & $\ldots$ \\
\hline Fibonacci & 2 & 3 & 5 & 8 & 13 & 21 & 34 & 55 & $\ldots$ \\
\hline \multirow[t]{2}{*}{ Lucas } & 3 & 4 & 7 & 11 & 18 & 29 & 47 & 76 & $\ldots$ \\
\hline & 4 & 5 & 9 & 14 & 23 & 37 & 60 & 97 & \\
\hline 0 & 1 & 2 & 3 & 5 & 8 & 13 & 21 & 34 & $\ldots$ \\
\hline 1 & 2 & 3 & 5 & 8 & 13 & 21 & 34 & 55 & $\ldots$ \\
\hline 2 & 3 & 4 & 7 & 11 & 18 & 29 & 47 & 76 & $\ldots$ \\
\hline 3 & 4 & 5 & 9 & 14 & 23 & 37 & 60 & 97 & $\ldots$ \\
\hline 4 & 5 & 6 & 11 & 17 & 28 & 45 & 73 & 118 & $\ldots$ \\
\hline
\end{tabular}

Figure D.1. The "golden" series: all the number series which are not crossed out correspond with the Golden Mean (Golden section). 


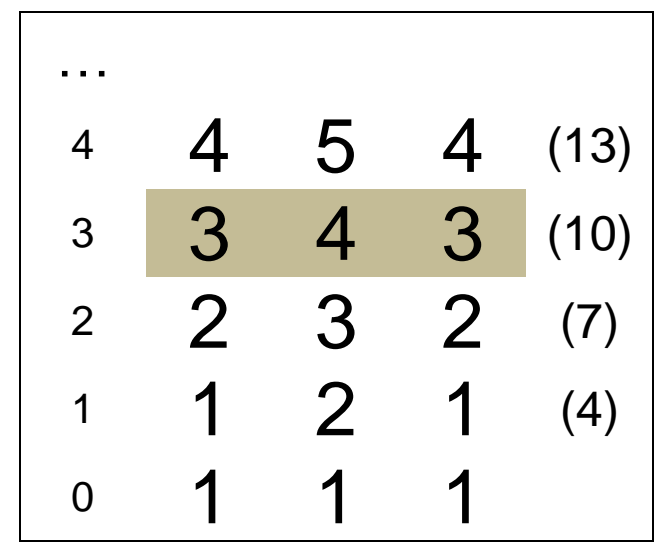

Figure D.2. The "evolution" of a triadic Cantor set (the simplest possible fractal), placed in the zeroth position; the evolution through the divergence for one unit in all three positions. From the first position onwards there is an "Extended triadic Cantor set" through the number of quantities at levels. Here a paradoxical situation becomes obvious: the farther we move from the beginning, the closer to it we get!? The biological meaning could be this: after a million years since the origin of life on Earth there were a lot of different species of organisms, but one and the same genetic code; after a hundred million years even a greater number and a greater variety of the species existed and the code remains the same; After a billion years everything is still enormously increased, but the code remained the same. The third case (dark tones) corresponds with the splitting of levels into Darwin's diagram, presented in Table 4.3. Also, the sums designated on the right of Figure (4-7-10-13- ...) correspond with the Shcherbak's numbering systems: The analogs to "Prime quantum 037 " have the numbering systems with the basis $q=4$, $7,10,13, \ldots$. 


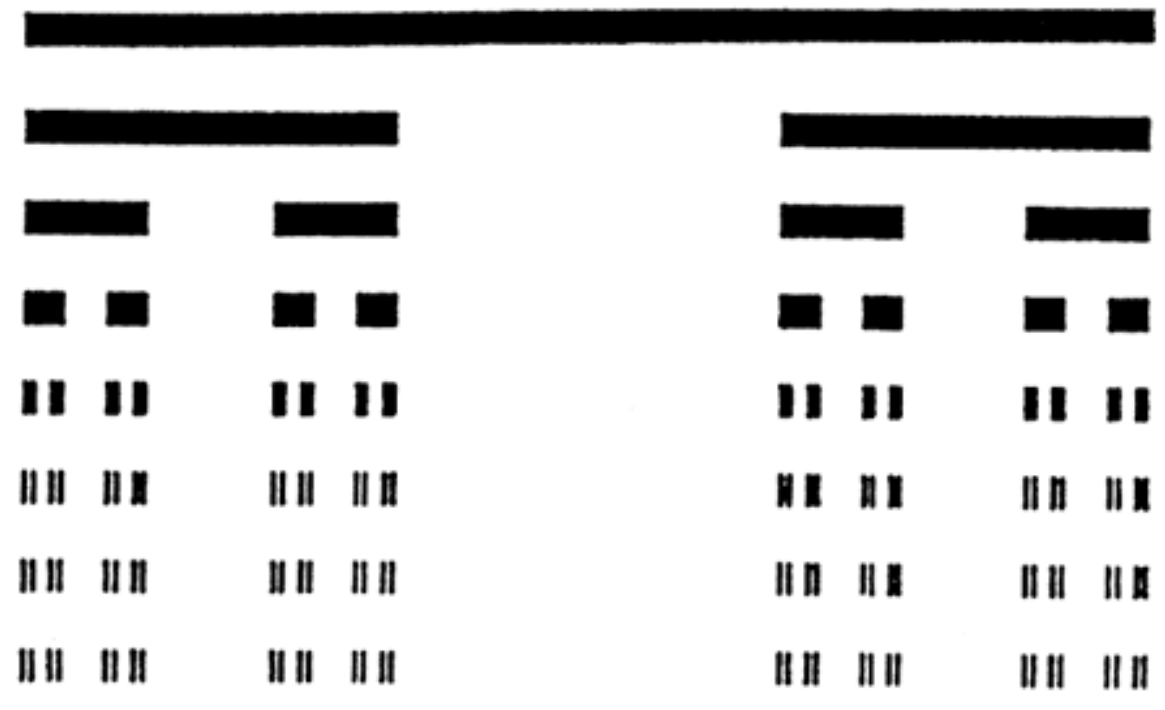

Figure D.3. The visualization of the Cantor triadic set as an infinite binary tree. 


\section{Appendix E}

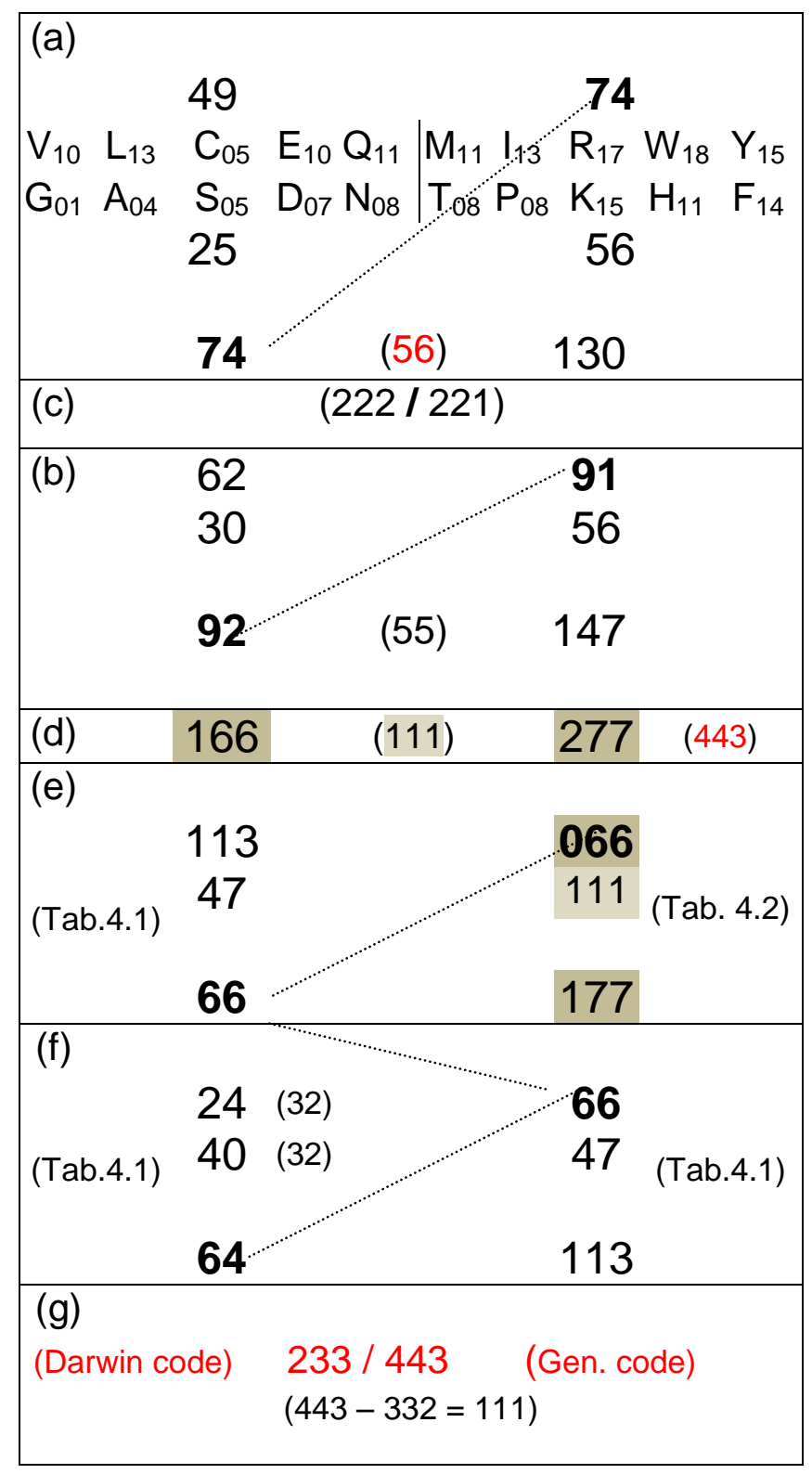

Table E.1. (a) The first class of AAs is in the upper row, and in the lower row there is the second class (Rakočević, 1997a): "Two classes of amino acids handled by two classes of enzymes. (Class II with 81 and Class I with 123 atoms.) The ten amino acid pairs, natural pairs from the chemical aspect, are classified into two classes. Class I contains larger amino acids (larger within the pairs), all handled by class I of enzymes aminoacyl-tRNA synthetases. Class II contains smaller amino acids, all handled by class II of synthetases. ... The order follows the number of atoms within side chains of class II AAs (given here as index); from left to right: first there are aliphatic, and then aromatic AAs. ... [Notice that the pair F-Y is simpler as only aromatic and $\mathrm{H}-\mathrm{W}$ is more complex as aromatic heterocyclic.]" (Rakočević, 2011, Table 2.1). Shcherbak's account of nucleon number within the amino acid constituents 
of GC, in their side chains (Figure 1.1) is as follows: $[\mathbf{1} \times(\mathrm{G} 1+\mathrm{A} 15+\mathrm{P} 41+\mathrm{V} 43+\mathrm{T} 45+\mathrm{C} 47+\mathrm{I} 57+\mathrm{N} 58$ $+\mathrm{D} 59+\mathrm{K} 72+\mathrm{Q} 72+\mathrm{E} 73+\mathrm{M} 75+\mathrm{H} 81+\mathrm{F} 91+\mathrm{Y} 107+\mathrm{W} 130)]+[2 \times(\mathrm{S} 31+\mathrm{L} 57+\mathrm{R} 100)]=1443$. If Shcherbak's account is done, with an iteration more, for the number of atom, the result is as follows: $2 \mathrm{x}$ $(\mathrm{G} 1+\mathrm{A} 4+\mathrm{C} 5+\mathrm{D} 7+\mathrm{N} 8+\mathrm{T} 8+\mathrm{P} 8+\mathrm{E} 10+\mathrm{V} 10+\mathrm{Q} 11+\mathrm{M} 11+\mathrm{H} 11+\mathrm{I} 13+\mathrm{F} 14+\mathrm{Y} 15+\mathrm{K} 15+$ $\mathrm{W} 18)]+[3 \times(\mathrm{S} 5+\mathrm{L} 13+\mathrm{R} 17)]=0443$ (here: row $d)$. On the other hand, within Darwin's diagram there are the next "branch" entities/quantities: 276 branches plus 46 nodes +10 branchings, in total 332. The significant differences are as follows: $1443-332=1111$ and 443-332 $=111$, both determined by the unity change law (here: row $g$ ); (b) Atom number within 23 amino acid molecules as in (a), except that twomeaning AAs (L,S,R) participate twice in the account: $204+35=239=92+147$. (c) The result of the „crossing“ sumation: $74+147=221$ and $130+92=222$; (d) The result of two sumation: $74+92=166$ and $130+147=277$; (d) The sumation of two sumations: $166+277=443$; (e) The results from Darwin's diagram as in Tables 4.1 and 4.2; (f) The results from Darwin's diagram $(66-64=\underline{\mathbf{2}})$ as in Tables 4.1 in correspondence with two results in genetic code: $92-91=\underline{\mathbf{1}}$ and $74-74=\underline{\mathbf{0}}$; (g) Final result in GC (443) in relation to the final Darwin's result (233), taken from Tables 4.5 and 5. 
Appendix F. A simple syllogism

1. Darwin's diagram corresponds with the presented arithmetical / algebraic systems

2. Genetic code corresponds with presented arithmetical / algebraic systems

3. Therefore, Darwin's diagram corresponds with the Genetic code

\begin{tabular}{|c|c|c|c|c|c|}
\hline \multicolumn{4}{|c|}{ Distrib. of AAs after Cloister energy and atom number } & Relations & \multirow{2}{*}{$\frac{\text { Chemical pairs }}{(\mathrm{H}-\mathrm{W})}$} \\
\hline & $\underline{\mathbf{H}} \quad 0.00 \quad 1.46 \underline{\mathbf{K}}$ & & H $0.001 .46 \mathrm{~K}$ & & \\
\hline 44 & A $-0.09 \quad 0.91 Q 60$ & 57 & A $-0.09 \quad 0.91 Q 68$ & & $(A-G)$ \\
\hline 45 & $\underline{\mathbf{G}}-0.160 .87 \underline{\mathbf{R}} 45$ & 54 & G -0.160 .87 R 54 & & $\mathrm{~V}-\mathrm{L}$ \\
\hline 89 & $\overline{\mathrm{W}}-0.25 \quad 0.71 \overline{\mathrm{E}} \mathbf{1 0 5}$ & 111 & W -0.25 0.71 E 122 & & $(\mathrm{~K}-\mathrm{R})$ \\
\hline & $\underline{\mathbf{V}}-0.520 .69 \underline{\mathbf{D}}$ & & $\mathrm{V}-0.520 .69 \mathrm{D}$ & $(44+44=88)^{49}$ & $\mathrm{Q}-\mathrm{E}$ \\
\hline \multicolumn{2}{|r|}{194} & & $\mathrm{~L}-0.54 \quad 0.52 \mathrm{~N}$ & $(60+56=116)^{50}$ & $\mathrm{D}-\mathrm{N}$ \\
\hline & $\mathrm{L}-0.54 \quad 0.52 \mathrm{~N}$ & \multicolumn{2}{|r|}{$(233)^{51}$} & & \multirow[b]{2}{*}{$(\mathrm{I}-\mathrm{P})$} \\
\hline 56 & $\underline{\mathbf{I}}-0.56 \quad 0.46 \underline{\mathbf{P}} 44$ & & I $-0.56 \quad 0.46 \mathrm{P}$ & & \\
\hline 45 & $\begin{array}{lllll}\mathrm{F} & -0.56 & 0.42 & \mathrm{Y} & 45\end{array}$ & 43 & F $-0.56 \quad 0.42$ Y 36 & & $\mathrm{~F}-\mathrm{Y}$ \\
\hline 101 & $\underline{\mathbf{M}}-0.57 \quad 0.27 \underline{\mathbf{T}} 89$ & 36 & M $-0.57 \quad 0.27$ T 36 & & $(\mathrm{M}-\mathrm{T})$ \\
\hline & $\mathrm{C}-0.73 \quad 0.24 \mathrm{~S}$ & & $\mathrm{C}-0.73 \ldots .24 \mathrm{~S}^{72}$ & & $\mathrm{C}-\mathrm{S}$ \\
\hline \multicolumn{2}{|r|}{190} & \multicolumn{2}{|r|}{151} & $\begin{array}{l}125(102+23) \\
79(102-23)\end{array}$ & $\begin{array}{l}125=57+68 \\
79=43+36\end{array}$ \\
\hline \multirow{2}{*}{\multicolumn{2}{|c|}{$\begin{array}{rlll}\text { Odd } & 46 & (102-1) & 55 \\
\text { Even } & 54 & (102+1) & 49\end{array}$}} & \multirow{2}{*}{\multicolumn{2}{|c|}{$\begin{array}{l}102 \pm x(\text { For } x=23 \text { we have the } \\
\text { correspondence with } 276)\end{array}$}} & & \\
\hline & & & & & \\
\hline
\end{tabular}

Table F.1. Distribution of amino acids after Cloister energy (Swanson, 1984) and atom number

[Note F.1. The chemical pairs after (Dlyasin, 1998, 2011; Rakočević, 1998, Survey 4, p. 290; Rakočević, 2004, Figures 1 and 2, p. 222). The pairs G-A and V-L as well as S-T and C-M after Dlyasin; in a vice versa logic: G-V and A-L as well as S-C and T-M after Rakočević; all other is the same].

\footnotetext{
${ }^{49}$ The connection with the number 276 through the relation: $\varphi(276)=88(088+188=276)$

${ }^{50}$ The connection with the number 60 and 56 through the relation: $\varphi(116)=56(56+60=116)$; the $\mathbf{5 6}$ as all primary branches at 1-10 levels as well as at 11-14 levels, for two species A and I (Tab. 2.1); the 60 as total number of all branches in upper part of Darwin Diagram (DD): the 56 as said, plus 4 branches in second set of species (9 species) as it is shown in (Table 6.1). [Note: in Table 6.1 see above the levels 10-14 with only 4 branches.]; the $\mathbf{1 1 6}$ as complement of 216 (footnotes 55 and 58).

${ }^{51}$ The 233, as all branches (prim. + second.) for two species, "A" and "I" into (3 x 5) levels (Tab. 4.5). Here: $111+$ 122 equals 233. In DD: 112 as all primary branches +121 as all secondary branches equals 233 .
} 


$(111+01=\mathbf{1 1 2})^{52} ;(122-01=\mathbf{1 2 1})^{53} ;\left[89+89=178 ;(178-01=\mathbf{1 7 7})^{54}\right.$
$\left(101+105=206(206+10=\mathbf{2 1 6})^{55} ;\right.$
$(2 \underline{\mathbf{0}} 6+17 \underline{\mathbf{8}}=384)^{56}$
$(2 \underline{\mathbf{1}} 6+17 \underline{\mathbf{7}}=393) ; \quad(384+393=777)^{57}$
$(116+\mathbf{2 1 6}=\mathbf{3 3 2}) ;^{58}(\mathbf{8 8}+\mathbf{1 8 8}=\mathbf{2 7 6})^{59}$
$(190-151=\mathbf{3 9})^{60}$
$(233-194=\mathbf{3 9})$

Survey F.1. Relations between quantitatives of Genetic code and existing quantitatives within Darwin's diagram (I)

\begin{tabular}{|l|l|l|}
\hline$(194-151=43)^{61}$ & $44+44=\mathbf{8 8}$ & {$\left[(233)+(43)^{62}=(276)\right]$} \\
$(233-190=43)$ & $60+56=\mathbf{1 1 6}$ & \\
\hline
\end{tabular}

Survey F.2. Relations between quantitatives of Genetic code and existing quantitatives within Darwin's diagram (I)

\footnotetext{
${ }^{52}$ The $\mathbf{1 1 2}$ as the number of all primary branches for two species, "A" and "I" into $(3 \times 5)$ levels (Tab. 2.1).

${ }^{53}$ The $\mathbf{1 2 1}$ as all secondary branches for two species, "A" and "I", into (1-10) levels (Table 2.2).

${ }^{54}$ The $\mathbf{1 7 7}$ as all branches (primary + secondary) for two species, "A" and "I" into (1-10) levels (Tab. 4.1). xxx

${ }^{55}$ The $\mathbf{2 1 6}$ as all branches (primary + secondary) for all 11 species at 1-10 levels (Table 7.2). xxx

${ }^{56}$ The $\mathbf{3 8 4}$ as total number of atoms in 20 amino acid molecules, within their "bodies" and "heads".

${ }^{57}$ Cf. with the starting 777 in Table B.2.

${ }^{58}$ The $\mathbf{2 1 6}$ as in footnote 55; then the $\mathbf{1 1 6}$ contains all other quantitatives to the sum of 332 (Table 5) "branch" quantitatives: 60 branches at 11-14 levels into all 11 species, plus 46 nodes (Table 3.1), plus 10 branchings (Table 3.2) [Note: the nodes and branchings exist only in species, "A" and "I".]

${ }^{59}$ The $\mathbf{8 8}$ as all primary branches for all 11 species at $1-10$ levels (Table 7.5). The $\mathbf{1 8 8}$ as the sum of all other branches to the total sum of $\mathbf{2 7 6}$.

${ }^{60}$ The 39 as all branches (primary + secondary) for 9 species (B, C, D, E, F on the left and G, H, K, L on the right) at 0-9, i.e. 1-10 levels (Table 6.5).

${ }^{61}$ The $\mathbf{4 3}$ as all branches (primary + secondary) for 9 species (B, C, D, E, F on the left and G, H, K, L on the right) at 0 -14 levels (Table 6.4).

${ }^{62}$ The $\mathbf{4 3}$ as in footnote 61.
} 
Appendix G. The number of hydrogen bonds within the set of four nucleotides

Why 2-3 and not 1-2 hydrogen bonds within the set of four nucleotides (UA connected with two and CG with three hydrogen bonds)? The answer follows from the relationships presented in Tables G.1 and G.2. If we have the alphabet of four letter (UCAG in Table G.1), then there are six their pairs (UC, AG, UA, CG, UG, CA). Also there are two possibilities for bonding (Tables G.1 \& G.2). Going from the arrangement in Table G.1 to the arrangement in Table G.2 the pairs $\mathrm{UG}, \mathrm{CA}$ appear to be invariant, but other four (two and two: UC/AG and UA/CG) variant. By this, from the chemical aspect we must speak: $\underline{\mathbf{2}}$ original pairs (UC/AG or UA/CG), $\underline{\mathbf{6}}$ derived pairs (UC, AG, UA, CG, UG, CA), $\underline{\mathbf{1 0}}$ hydrogen bonds $(5+5$ or $4+6)$. Altogether this is the correspondence with the Hückel's formula, in form $\mathrm{N}=2(2 \mathrm{n}+1)(\mathrm{n}=0,1,2,3)$ as it is presented in Section 4.3 and Box 2. In Table G.1 we can find this arrangement only it the case with 2-3 hydrogen bonds, in wich case the principles of continuity and minimum change are also valid.

One must notice that the pattern 4-5-5-6 of hydrogen bonds corresponds with the same system existing within Rumer's Table of nucleotide doublets. (Cf. Tables 1 \& 2 in Book of Abstracts - Theoretical Approaches to Bioinformation Systems, TABIS 2013, 17-22 September 2013, Belgrade, Serbia.) (Proceedings in press.) Notice also that this pattern corresponds with the 4-5-5-6 amino acid pairs, presented in this paper in Table F.1. 


\begin{tabular}{|c|c|c|}
\hline CG & 8 & \\
\hline CA & 7 & 14 \\
\hline UG & 7 & 14 \\
\hline UA & 6 & \\
\hline- & - & \\
\hline- & - & \\
\hline - & $\overline{-}$ & \\
\hline- & - & \\
\hline- & - & \\
\hline- & & \\
\hline
\end{tabular}
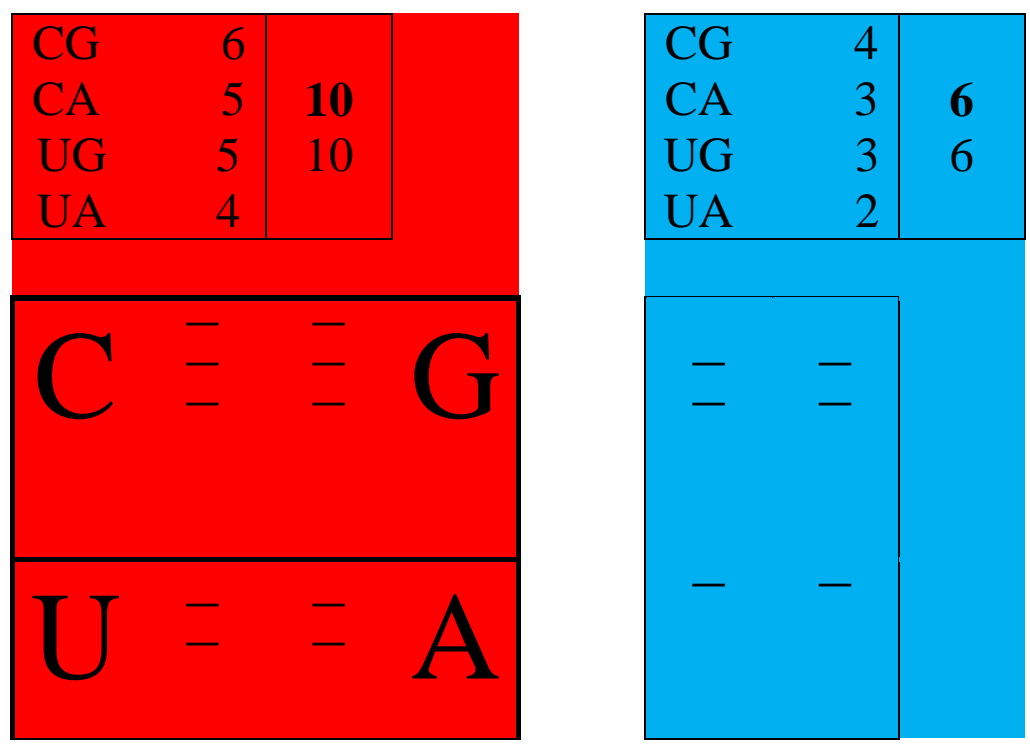

$$
\begin{aligned}
& \mathrm{N}=2(2 \mathrm{n}+1) \\
& \mathrm{N}=2,6,10,14 \ldots
\end{aligned}
$$

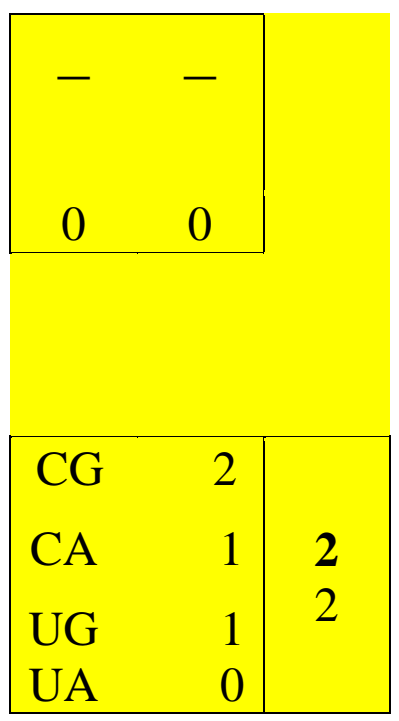

Table G.1. The number of real and hypothetical hydrogen bonds (I) 

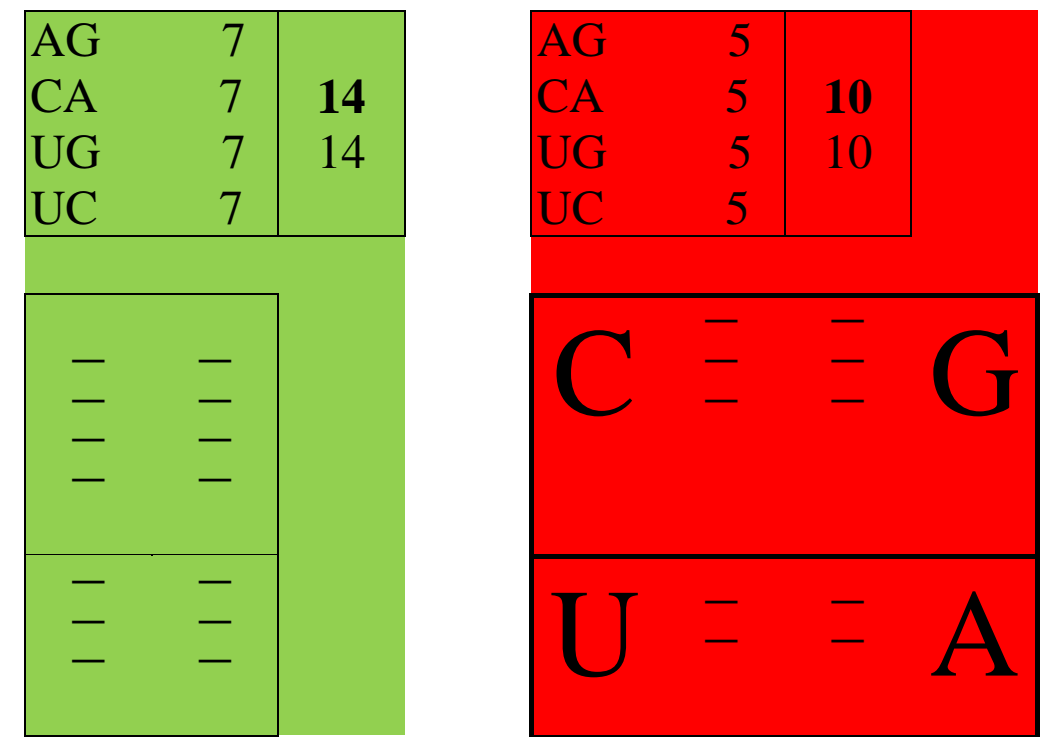

$$
\begin{aligned}
& \mathrm{N}=2(2 \mathrm{n}+1) \\
& \mathrm{N}=2,6,10,14 \ldots
\end{aligned}
$$
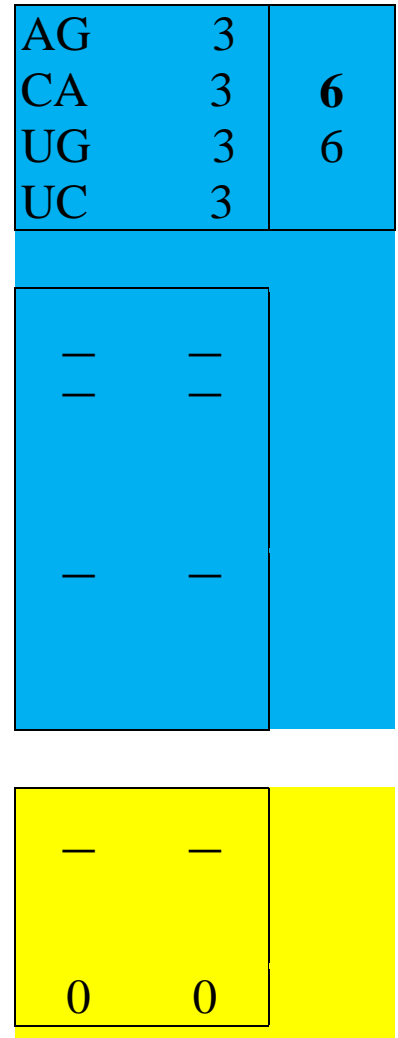

\begin{tabular}{|ll|l|}
\hline AG & 1 & \\
CA & 1 & $\mathbf{2}$ \\
UG & 1 & 2 \\
UC & 1 & \\
\hline
\end{tabular}

Table G.2. The number of real and hypothetical hydrogen bonds (II) 
Appendix H. Some citations from Darwin's book

[Darwin, Ch. (1876) The Origin of Species, John Murray, 6th ed., with additions and corrections, London]

Citation 1a (p. 90). The accompanying diagram ...

The accompanying diagram will aid us in understanding this rather perplexing subject. Let $\mathrm{A}$ to $\mathrm{L}$ represent the species of a genus large in its own country; these species are supposed to resemble each other in unequal degrees, as is so generally the case in nature, and as is represented in the diagram by the letters standing at unequal distances. I have said a large genus, because

“... letters ... distances” (Explanation 1a)

Explanation 1a. It is clear that Darwin here insists on the letters as such. In this case in the question are large letters below, under the zeroth level.

\section{Citation 1b (p. 92). In our diagram ...}

and the more their moditied progeny will increase. In our diagram the line of succession is broken at regular intervals by small numbered letters marking the successive forms which have become sufficiently distinct to be recorded as varieties. But these breaks are imaginary, and might have been inserted anywhere, after intervals long enough to allow the accumulation of a considerable amount of divergent variation.

“... by small numbered letters .... as varieties” (Explanation 1b)

Explanation 1b. At the levels 1-10 all varieties are designated "by small numbered letters". However, at the levels 11-14, only varieties at the level 14 are with letters and other ( $3 \times 14=42$ varieties) at 11-13 levels are without letters (see Citations $2 \& 3$ ).

Citation 2 (p. 91): The intervals ...

The intervals between the horizontal lines in the diagram, may represent each a thousand or more generations. After a thousand generations, species (A) is supposed to have produced two fairly well-marked varieties, namely $a^{1}$ and $m^{1}$. These two varieties will

“After a thousand generations ... well-marked varieties" (Explanation 2) 
Explanation 2. Varieties as the primary branches, and as the letters to the 10th level, at the same time; well-marked also at the level 14. H.1

Citation 3 (p. 91): In the diagram ... a condensed and simplified form

and some failing to produce any. Thus the varieties or modified descendants of the common parent (A), will generally go on increasing in number and diverging in character. In the diagram the process is represented up to the ten-thousandth generation, and under a condensed and simplified form up to the fourteen-thousandth generation.

“... to the fourteen-thousand generation" (Explanation 3)

Explanation 3. If "to the fourteen", then that means at $11^{\text {th }}, 12^{\text {th }}$ and the $13^{\text {th }}$ at the same time.

From this small discussion it follows: from all primary branches (varieties) in two species (,A“ and „I“), them 112, organized in form of two trees, and presented in Table 2.1, the 70 (5 x 14) are ,well-marked" by 70 letters and 42 (3 x 14) are not marked with the letters. (The 56 primary branches on the levels I-X, marked with the letters, plus 14 on the $14^{\text {th }}$ level, equals 70 ,well-marked" primary branches; then $70+42=112$.)

On the other hand, from all primary branches (varieties) in remaining 9 species, them 36, organized without of two trees, and presented in Table 6.1, the 3 (1 x 3) are well-marked by 3 letters (E \& F at the $10^{\text {th }}$ level and $\mathrm{F}$ at $14^{\text {th }}$ level) and $33(11 \times 3)$ are not marked with the letters. Altogether, $70+3=73$ well-marked and $42+33=075$ not marked $(75+73=148$ as in Table 7.3). (Cf. first two cases in last columns of Tables H.1, H.2 and H.3). By this one must notice that the result 075 corresponds with the middle position in Table H.1 (ㅁ5 vs $\underline{185}$ ). Also, from these relationships it follows that the number " 73 " we can read as 73 branches and as 73 letters. Analogously with Shcherbak's method (L, S, R two times in calculation) it makes sense to have the sum of all branch-letters entities, that means: $73+148=\mathbf{2 2 1}$, which result we have in the GC (Table E.1, a,b,c: $147+74=221$ and $92+130=222$ ), where Shcherbak's method (with one iteration more) also acts. On the other hand, the 73 well-marked primary branches and 75 nonmarked, appear to be in the ratio 74 \pm 1 (Table H.3), what a balance is and where the 74 is also a Shcherbak's number as the number of nucleons within the amino acid "head".

In addition to the said: there is a whole Darwin Diagram Space, i.e. the Darwin Diagram System (DDS), consisting of "tree-space” (left tree „A“ \& right tree „I“) and "non-tree-space” [left part (B,C,D,E,F) \& right part $(\mathrm{G}, \mathrm{H}, \mathrm{K}, \mathrm{L})]$. Within whole DDS there are 73 letters, and within "tree-space" there are 112 primary branches (Table 2.1). From this facts, it makes sense to calculate the sum $73+112=185$. Why? Because the 73 are branch-letters in whole DD system, 
and 112 are branches in DD sub-system; the sub-system as a „half“ of whole system. [,Whole“ and "half" as a condition for the realization of the "symmetry in the simplest case" (Marcus, 1989, p. 103).]

And now, as we see from Table H.1, the number of 185 ,branch“ entities, appers to be in the middle position within a specific arithmetical system, the system of relationships between multiples of second perfect number 28 and Shcherbak's „Prime quantum 37“.

Within whole DDS there are 148 primary branches (Table 7.3), and within "tree-space" (as a „half" of „whole“) there are 70 letters (73 of total letters minus E \& F on the 10th and F on the 14th level); the sum of 148 primary branches in both spaces (tree-space and non-tree-space as a whole) plus 70 letters in tree-space (as a ,half") $=218$; these 218 quantitatives (as a new whole), minus 33 primary non-marked branches (as a new "half") equals 185 once more. (Note: The 33 primary non-marked branches we have within "non-tree-space", if we subtract 3 from 36 in Table 6.1; these 3 are - the 3 well-marked primary branches, designated with $\mathrm{E} \& \mathrm{~F}$ at 10th and $\mathrm{F}$ at 14th level.)

\begin{tabular}{|c|c|c|c|c|c|c|}
\hline & Multiples of 1 & & Sums & \multicolumn{3}{|c|}{ Double values } \\
\hline 1 & $14 \quad$ (23) & 37 & 51 & 28 & (46) & 74 \\
\hline 2 & (46) & 74 & 102 & 56 & (92) & 148 \\
\hline 3 & (69) & 111 & 153 & 84 & (138) & 222 \\
\hline 4 & (92) & 148 & 204 & 112 & (184) & 296 \\
\hline 5 & (115) & 185 & 255 & 140 & (230) & 370 \\
\hline 6 & (138) & 222 & 306 & 168 & (276) & 444 \\
\hline 7 & (161) & 259 & 357 & 196 & (322) & 518 \\
\hline 8 & $(184)$ & 296 & 408 & 224 & (368) & 592 \\
\hline 9 & (207) & 333 & 459 & 252 & (414) & 666 \\
\hline
\end{tabular}

Table H.1. The arithmetical relationships between second perfect number 28 and Shcherbak's "Prime quantum 37". There are some GC significant numbers: 204 as the number of atom within 20 amino acid side chains; the number $\underline{\mathbf{0}} 255$ corresponds with the total number (1255) of nucleons within 20 AA side chains; the number 74 as the number of nucleon within AA "head". (Notice an analogy between this pattern $\underline{\mathbf{0}} 255$ / 1255 and the pattern $\underline{\mathbf{0}} 443$ / 1443 in Table E.1, valid for the number of atoms and nucleons, respectively.) 


\begin{tabular}{|c|c|c|c|c|c|c|c|}
\hline Tab. H.2 & 1 & 2 & 3 & & 5 & 6 & $3+6$ \\
\hline 1 & $(24$ & 18 & $42)^{63}$ & $(24$ & 09 & $33)^{64}$ & $75^{65}$ \\
\hline II & (44 & 26 & $70)^{66}$ & (03 & 00 & $03)^{6 /}$ & $73^{68}$ \\
\hline I+II & (68 & 44 & $(112)^{69}$ & (27 & 09 & $36)^{/ 0}$ & $148^{/ 1}$ \\
\hline III & (77 & 44 & $121)^{72}$ & (03 & 04 & $07)^{73}$ & $128^{74}$ \\
\hline$|+| I+|I|$ & (145 & 88 & $233)^{1 / 3}$ & (30 & 13 & $43)^{16}$ & $276^{\prime \prime}$ \\
\hline
\end{tabular}

Table H.2. Distributions and Distinctions within Darwin Diagram, DDDD-1: "tree-space" $(1,2,3)$ versus "non-tree-space" $(4,5,6)$ on 0-14 levels; I.1: The left tree-space ("A"): the 24 non-marked primary

${ }^{63}$ The results 24-18-42 (non-marked primary branches on 11-13th levels) (non-marked by the small letters) in relation to total primary branches on both trees (A \& I): 68-44-112 (Table 2.1).

64 The results 24-09-33 (non-marked primary branches on 0-14th levels) (non-marked by the small letters) in relation to total primary branches at the left and right non-tree-space, respectively (left: B,C,D,E,F; right: G,H,K,L): 27-09-36 (Table 6.1).

${ }^{65}$ The total number of non-marked primary branches (varieties non-designated with small letters).

${ }^{66}$ The results 44-26-70 as well-marked primary branches within tree-space, on the trees "A" and "I" (Table 2.1 and Figure 5).

67 The results 03-00-03 as well-marked primary branches within non-tree-space (only within the left space: B,C,D,E,F; that, because within the right space: G,H,K,L the well-marked primary branches do not exist (Table $6.1)$.

${ }^{68}$ The total number of well-marked primary branches (varieties designated with small letters).

${ }^{69}$ The total number of primary branches within tree-space, on the trees "A" and "I" (Table 2.1 and Figure 5).

${ }^{70}$ The total number of primary branches within non-tree-space (left: B,C,D,E,F; right: G,H,K,L) (Table 6.1). At the same time e: The Darwin's equation as in Table 6.1 and in Figure 6.

71 The total number of primary branches within tree-space ("A" \& "I") and non-tree-space (left: B,C,D,E,F; right: $\mathrm{G}, \mathrm{H}, \mathrm{K}, \mathrm{L})$. The number 148 together with the subsequent number below (128) appears to be a change for \pm 10 in relation to the half of number 276 as total number of branches within the Diagram $(2 \times 138=276)(\mathrm{cf}$. Table 7.3 with Table 7.1).

72 The total number of secondary branches (non-varieties) (Table 2.2 and Figure 4).

73 All secondary branches for 9 species (B, C, D, E, F on the left and G, H, K, L on the right) at 0-9 levels (Table 6.3).

74 The total number of secondary branches within tree-space ("A" \& "I") and non-tree-space (left: B,C,D,E,F; right: $\mathrm{G}, \mathrm{H}, \mathrm{K}, \mathrm{L}$ ) (Table 6.1). The number 128 together with the subsequent number above (148) appears to be a change for \pm 10 in relation to the half of number 276 as total number of branches within the Diagram $(2 \times 138=276)(\mathrm{cf}$. Table 7.4 with Table 7.1). The number 128 as the sum of $121+7$.

75 The total number of branches (primary plus secondary) within tree-space ("A" \& "I") (Table 4.5 \& Table 5; both in relation to Table E.1, g).

${ }^{76}$ The total number of branches (primary plus secondary) within non-tree-space (left: B,C,D,E,F; right: G,H,K,L) at 0-14 levels (Table 6.4).

77 The total number of branches (primary plus secondary) within tree-space ("A" \& "I") and non-tree-space (left: B,C,D,E,F; right: G,H,K,L) (Table 7.1). The 276 as the sum of $233+43$. 
branches $(3 \times 8=24)$ on 11-13th levels; I.2: The right tree-space ("I"): the 18 non-marked primary branches $(3 \times 6=18)$ on 11-13th levels; I.3: The sum of previous two (Table 2.1); I.4: The left non-treespace (B,C,D,E,F): the 24 non-marked primary branches (21 on the levels $0-9$ and 3 on the levels 11-13); I.5: The right non-tree-space $(\mathrm{G}, \mathrm{H}, \mathrm{K}, \mathrm{L})$ : the 9 non-marked primary branches (all 9 on the levels 0-5); I.6: The sum of previous two (Table 6.1); II.1: The left tree-space ("A"): the 44 well-marked primary branches (36 on the levels $0-9$ and 8 on the level 14); II.2: The right tree-space ("I"): the 26 well-marked primary branches (20 on the levels $0-9$ and 6 on the level 14); II.3: The sum of previous two (Table 2.1); II.4: II.4: The left non-tree-space (B,C,D,E,F): the 3 well-marked primary branches (E \& F with the start on the level $9^{\text {th }}$ and the finalization on the level $10^{\text {th }}$; plus $\mathrm{F}$ with the start on the level $13^{\text {th }}$ and the finalization on the level $14^{\text {th }}$ ); II.5: The right non-tree-space (G,H,K,L): without well-marked primary branches; II.6: II.6: The sum of previous two (Table 6.1). With all these facts it must be noted that the vertically shaded field is in connection with arithmetical systems in Figures 4 \& 5, and the horizontally shaded field with the Darwin's equation, observed in Table 6.1 and presented in Figure 6.

\begin{tabular}{|c|ccc|c|ccc|c|c|}
\hline Tab.H.3 & 1 & 2 & 3 & & 4 & 5 & 6 & $3+6$ & \\
\hline I & 24 & 24 & $\mathbf{4 8}$ & & 18 & 09 & $\mathbf{2 7}$ & 75 & \multirow{2}{*}{ II } \\
44 & 03 & $\mathbf{4 7}$ & & 26 & 00 & $\mathbf{2 6}$ & 73 & \pm 74 \\
I+II & 68 & 27 & 95 & & 44 & 09 & 53 & 148 & \\
III & 77 & 03 & 80 & & 44 & 04 & 48 & 128 & \\
I+II+III & 145 & 30 & 175 & & 88 & 13 & 101 & 276 & \\
\hline
\end{tabular}

Table H.3. Distributions and Distinctions within Darwin Diagram, DDDD-2: Left part of Darwin Diagram (DD) versus Right part, 1-14 levels. All data in columns 1, 2, 4, 5 are the same as in Table H.2, except the interchange of two columns: $2 \& 4$, respectively. The horizontally shaded fields correspond with the Darwin's equation as it is presented in Tables H.4 and H.5; The vertically shaded field is in connection with a unique situation in a specific arithmetical system, presented in Table H.6.

\begin{tabular}{|l|lll|l||ccc|ccc|c|}
\hline \multicolumn{5}{|c|}{ Table H.4 } & -09 & & -09 & -18 & & -18 & \\
\hline 1 & 09 & 09 & 18 & 2 \\
2 & 18 & 09 & 27 & 3 \\
3 & 27 & 09 & 36 & 4 & 00 & 09 & 09 & -09 & 09 & 00 & $\ldots$ \\
09 & 09 & 18 & & 00 & 09 & 09 & \\
4 & 36 & 09 & 45 & 5 & 18 & 09 & 27 & 09 & 09 & 18 & \\
5 & 45 & 09 & 54 & 6 & 27 & 09 & 36 & 18 & 09 & 27 & \\
6 & 54 & 09 & 63 & 7 & 36 & 09 & 45 & 27 & 09 & 36 & \\
7 & 63 & 09 & 72 & 8 & 45 & 09 & 54 & 36 & 09 & 45 & \\
8 & 72 & 09 & 81 & 9 & 54 & 09 & 63 & 45 & 09 & 16 & $\ldots$ \\
9 & 81 & 09 & 90 & 10 & 09 & 72 & 54 & 09 & 36 & \\
& $\ldots 3$ & 09 & 81 & 63 & 09 & 52 & \\
& $\ldots$ & & & & & & & & & & \\
\hline
\end{tabular}

Table H.4. An arithmetical system of multiples of number 9 with an inclusion of Darwin's equation in the darker ton (Variant I). 


\begin{tabular}{|c|ccc|c|ccc|c|ccc|c|}
\hline \multicolumn{3}{|c|}{ Table H.5 } & & -1 & & -1 & -2 & & -2 & \\
\hline 1 & 09 & 09 & 18 & 2 & 08 & 09 & 17 & 07 & 09 & 16 & $\ldots$ \\
3 & 27 & 09 & 36 & 4 & 26 & 09 & 35 & & 25 & 09 & 34 & \\
5 & 45 & 09 & 54 & 6 & 44 & 09 & 53 & 43 & 09 & 52 & \\
7 & 63 & 09 & 72 & 8 & 62 & 09 & 71 & 61 & 09 & 70 & \\
9 & 81 & 09 & 90 & 10 & 80 & 09 & 89 & 79 & 09 & 88 & \\
& $\ldots$ & & & & & & & & & & & \\
\hline
\end{tabular}

Table H.5. An arithmetical system of multiples of number 9 with an inclusion of Darwin's equation in the darker ton (Variant II).

\begin{tabular}{|c|c|c|c|c|c|c|c|c|c|c|c|}
\hline 2,2,2 & 10 & 25 & 35 & 2,2,2 & 10 & 25 & 35 & 2,2,2 & 10 & 25 & 35 \\
\hline $2,2,2$ & 20 & 35 & 55 & $2,2,2$ & 20 & 35 & 55 & $2,2,2$ & 20 & 35 & 55 \\
\hline $2,2,2$ & 30 & 45 & 75 & $2,2,2$ & 30 & 45 & 75 & $2,2,2$ & 30 & 45 & 75 \\
\hline $2,2,2$ & 40 & 55 & 95 & 2,2,2 & 40 & 55 & 95 & 2,2,2 & 40 & 55 & 95 \\
\hline $2,2,3$ & 50 & 65 & 115 & $2,2,3$ & 50 & 65 & 115 & $2,2,3$ & 50 & 65 & 115 \\
\hline $2,2,3$ & 60 & 75 & 135 & $2,2,3$ & 60 & 75 & 135 & $2,2,3$ & 60 & 75 & 135 \\
\hline $2,2,3$ & 70 & 85 & 155 & $2,2,3$ & 70 & 85 & 155 & $2,2,3$ & 70 & 85 & 155 \\
\hline $2,2,3$ & 80 & 95 & 175 & $2,2,3$ & 80 & 95 & 175 & $2,2,3$ & 80 & 95 & 175 \\
\hline $2, \underline{\mathbf{3}}, 3$ & 90 & 105 & 195 & $2, \mathbf{3}, 3$ & $\underline{9} 0$ & 105 & $1 \underline{9} 5$ & $2, \mathbf{3}, 3$ & 90 & 105 & 195 \\
\hline$\underline{3}, 3,3$ & 100 & 115 & 215 & $\underline{\mathbf{3}}, 3,3$ & 100 & 115 & 215 & $\underline{\mathbf{3}}, 3,3$ & 100 & 115 & 215 \\
\hline $3,3,3$ & 110 & 125 & 235 & $3,3,3$ & 110 & 125 & 235 & $3,3,3$ & 110 & 125 & 235 \\
\hline $3,3,3$ & 120 & 135 & 255 & $3,3,3$ & 120 & 135 & 255 & $3,3,3$ & 120 & 135 & 255 \\
\hline $3,3,3$ & 130 & 145 & 275 & $3,3,3$ & 130 & 145 & 275 & $3,3,3$ & 130 & 145 & 275 \\
\hline \multirow[t]{5}{*}{$3,3,3$} & 140 & 155 & 295 & 3,3,3 & 140 & 155 & 295 & $3,3,3$ & 140 & 155 & 295 \\
\hline & & & & $3,3,3$ & 150 & 165 & 315 & $3,3,3$ & 150 & 165 & 315 \\
\hline & & & & $3,3,3$ & 160 & 175 & 335 & $3,3,3$ & 160 & 175 & 335 \\
\hline & & & & & & & & $3,3,3$ & 170 & 185 & 355 \\
\hline & & & & & & & & $3,3,3$ & 180 & 195 & 375 \\
\hline
\end{tabular}

Table H.6. A specific arithmetical system with two or three digit numbers. In relation to left and right area, the middle area appears to be unique: the central row (80-95-175) is in a direct connection with only one situation where appears a digit-triplet with the Gray code changes (2-2-3 / 2-י-3 / $\underline{\mathbf{3}}-3-3)$. Just this case is also the Darwin's case in third column of Table H.3. (Notice the correspondence with Table H.1 from the aspect of appearance of two-three digit numbers.) 
Appendix K. Another correspondence with the genetic code $e^{78}$

From Table K.1 follow Tables K.2 and K.3 with first half of AAs in first column and the second one in the second column. For each AA is given atom number in whole molecule. As we can see, atom number within 12 AAs in second column is 233 ; exactly as the number of all branches (primary plus secondary) in two trees, A \& I, within Darwin's diagram (Table 4.5); and in first column $233-1=232$.

\begin{tabular}{|c|c|c|c|c|c|c|c|c|c|}
\hline \multirow{2}{*}{$\begin{array}{l}1 \\
\text { lett }\end{array}$} & \multicolumn{8}{|c|}{ 2nd letter } & \multirow{2}{*}{$\begin{array}{l}\text { 3rd } \\
\text { lett. }\end{array}$} \\
\hline & \multicolumn{2}{|c|}{$\mathrm{U}$} & C & & A & & $\mathrm{G}$ & & \\
\hline $\mathrm{U}$ & 00. UUU & \multirow[t]{2}{*}{$\mathrm{F}$} & 08. UCU & \multirow{4}{*}{ S } & 32. UAU & \multirow[t]{2}{*}{ Y } & 40. UGU & \multirow[t]{2}{*}{$\mathrm{C}$} & $U$ \\
\hline \multirow{3}{*}{$\mathrm{I}$} & 01. UUC & & 09. UCC & & \multirow{3}{*}{$\begin{array}{l}\text { 33. UAC } \\
\text { 34. UAA } \\
\text { 35.UAG }\end{array}$} & & 41. UGC & & $C$ \\
\hline & 02. UUA & \multirow[t]{2}{*}{$\mathrm{L}$} & 10. UCA & & & \multirow[t]{2}{*}{ CT } & 42. UGA & CT & \multirow{2}{*}{$\begin{array}{l}A \\
G\end{array}$} \\
\hline & 03. UUG & & 11. UCG & & & & 43. UGG & W & \\
\hline \multirow{4}{*}{$\mathrm{C}$} & 04. CUU & \multirow{4}{*}{$\mathrm{L}$} & 12. CCU & \multirow{4}{*}{$\mathrm{P}$} & 36. CAU & & 44. CGU & \multirow{4}{*}{$\mathrm{R}$} & $U$ \\
\hline & 05. CUC & & 13. CCC & & 37. CAC & $\mathrm{H}$ & 45. CGC & & $C$ \\
\hline & 06. CUA & & 14. CCA & & 38. CAA & & 46. CGA & & $A$ \\
\hline & 07. CUG & & 15. CCG & & 39. CAG & Q & 47. CGG & & $G$ \\
\hline \multirow{4}{*}{ A } & 16. AUU & & 24. ACU & \multirow{4}{*}{$\mathrm{T}$} & 48. AAU & & 56. AGU & \multirow{2}{*}{ S } & $\mathrm{U}$ \\
\hline & 17. AUC & I & 25. ACC & & 49. AAC & $\mathrm{N}$ & 57. AGC & & $\mathrm{C}$ \\
\hline & 18. AUA & & 26. ACA & & 50. AAA & & 58. AGA & & A \\
\hline & 19. AUG & M & 27. ACG & & 51. AAG & $\mathrm{K}$ & 59. AGG & $\mathrm{K}$ & G \\
\hline \multirow{4}{*}{ G } & 20. GUU & & 28. GCU & \multirow{4}{*}{ A } & 52. GAU & & 60. GGU & \multirow{4}{*}{ G } & $U$ \\
\hline & 21. GUC & $v$ & 29. GCC & & 53. GAC & $D$ & 61. GGC & & $C$ \\
\hline & 22. GUA & V & 30. GCA & & 54. GAA & $F$ & 62. GGA & & $A$ \\
\hline & 23. GUG & $v$ & 31. GCG & & 55. GAG & $\mathrm{E}$ & 63. GGG & & $G$ \\
\hline
\end{tabular}

Table K.1. „The Table of the standard genetic code (GCT). Total codon space is divided into three parts in correspondence with the harmonic mean $(\mathrm{H})$ of the whole codon space sequence (a) and its half (b), where $\mathrm{a}=63, \mathrm{~b}=31.5$ and $\mathrm{H}=42 \ldots$ In the central area, the three stop codons (CT, codon terminations) are crossed out" (Rakočević, 2013, Table 1). By this one must noice now the correspondence and selfsimilarity between patterns of quantitives: $\underline{\mathbf{0}} 22$ / $\underline{\mathbf{0}} 21$ of codons in this Table versus $\underline{1} 22$ / $\underline{\mathbf{1}} 21$ of atoms in Table K.3.

\footnotetext{
${ }^{78}$ Appendices labeled with the letters "I" and "J" in this paper do not exist.
} 


\begin{tabular}{|c|c|c|c|c|c|c|c|}
\hline$F$ & 23 & 20 & $\mathrm{H}$ & $F$ & \multirow[b]{2}{*}{45} & \multirow[b]{2}{*}{40} & $\mathrm{H}$ \\
\hline $\mathrm{L}$ & 22 & 20 & Q & $\mathrm{L}$ & & & Q \\
\hline L & 22 & 17 & $\mathrm{~N}$ & L & \multirow[b]{2}{*}{44} & \multirow{2}{*}{41} & $\mathrm{~N}$ \\
\hline I & 22 & 24 & $\mathrm{~K}$ & I & & & $\mathrm{K}$ \\
\hline M & 20 & 16 & D & M & \multirow[b]{2}{*}{39} & \multirow[b]{2}{*}{35} & D \\
\hline V & 19 & 19 & $E$ & V & & & $E$ \\
\hline V & 19 & 14 & C & V & \multirow{3}{*}{33} & \multirow{2}{*}{41} & C \\
\hline S & 14 & 27 & W & $S$ & & & W \\
\hline$P$ & 17 & 26 & $\mathrm{R}$ & $P$ & & \multirow[b]{2}{*}{40} & $\mathrm{R}$ \\
\hline $\mathrm{T}$ & 17 & 14 & $S$ & $\mathrm{~T}$ & 34 & & S \\
\hline A & 13 & 26 & $\mathrm{R}$ & A & \multirow[b]{2}{*}{37} & \multirow{2}{*}{36} & $\mathrm{R}$ \\
\hline$Y$ & 24 & 10 & $\mathrm{G}$ & $Y$ & & & $\mathrm{G}$ \\
\hline & 114 & 119 & 233 & & \multirow{2}{*}{$\begin{array}{l}118 \\
114\end{array}$} & \multirow{2}{*}{$\begin{array}{l}115 \\
118\end{array}$} & 233 \\
\hline & 118 & 114 & 232 & & & & 232 \\
\hline & 232 & 233 & & & 232 & 233 & \\
\hline
\end{tabular}

Table K.2. The Table follows from Table 1: odd /even atom number distinctions.

\begin{tabular}{|c|c|c|c|c|c|}
\hline$F$ & 23 & 20 & $\mathrm{H}$ & \multirow{4}{*}{$\begin{array}{l}\text { (d) } 65 \\
63\end{array}$} & \multirow[b]{2}{*}{$-53(e)$} \\
\hline $\mathrm{L}$ & 22 & 20 & Q & & \\
\hline L & 22 & 17 & $\mathrm{~N}$ & & -63 \\
\hline I & 22 & 24 & K & & \\
\hline M & 20 & 16 & D & \multirow{2}{*}{\multicolumn{2}{|c|}{$244-233=0 \underline{11}$}} \\
\hline V & 19 & 19 & $E$ & & \\
\hline \multicolumn{4}{|c|}{$\begin{array}{l}\text { Crossing } \\
\text { Horizontal } \\
\text { Vertical }\end{array}$} & \multicolumn{2}{|c|}{$\begin{array}{l}128+1 \underline{1} 6=244(2 \times 122) \\
1 \underline{1} 8+1 \frac{2}{2} 6=244(2 \times 122) \\
1 \underline{1} 8+1 \underline{2} 6=244(2 \times 122)\end{array}$} \\
\hline V & 19 & 14 & $\mathrm{C}$ & \multirow{4}{*}{$\begin{array}{l}\text { (f) } 49 . \\
55 .\end{array}$} & \multirow{4}{*}{$-66(g)$} \\
\hline S & 14 & 27 & W & & \\
\hline $\mathrm{P}$ & 17 & 26 & $\mathrm{R}$ & & \\
\hline $\mathrm{T}$ & 17 & 14 & S & & \\
\hline A & 13 & 26 & $R$ & \multirow{2}{*}{\multicolumn{2}{|c|}{$\begin{array}{l}232-121=\frac{\mathbf{1 1 1}}{222} \\
100+122=2\end{array}$}} \\
\hline Y & 24 & 10 & G & & \\
\hline \multicolumn{4}{|c|}{$232 \quad 233$} & \multirow{2}{*}{\multicolumn{2}{|c|}{$\begin{array}{l}\mathrm{C} 100+121=221(1 \times 221) \\
\mathrm{H} 1 \underline{1} \overline{1}+1 \underline{0} 6=221(1 \times 221) \\
\mathrm{V} 1 \underline{\mathbf{0}} 4+1 \underline{1} \mathbf{1}=221(1 \times 221)\end{array}$}} \\
\hline \multicolumn{4}{|c|}{$233-232=00 \underline{1}$} & & \\
\hline & $232-$ & $=1$ & & \multicolumn{2}{|c|}{$\begin{array}{l}\frac{1}{0} 4+1 \underline{1}=221(1 \times 221) \\
384-100=284\end{array}$} \\
\hline
\end{tabular}

Table K.3. The Table follows from Table K.2. For details cf. Surveys K.1 and K.2. 


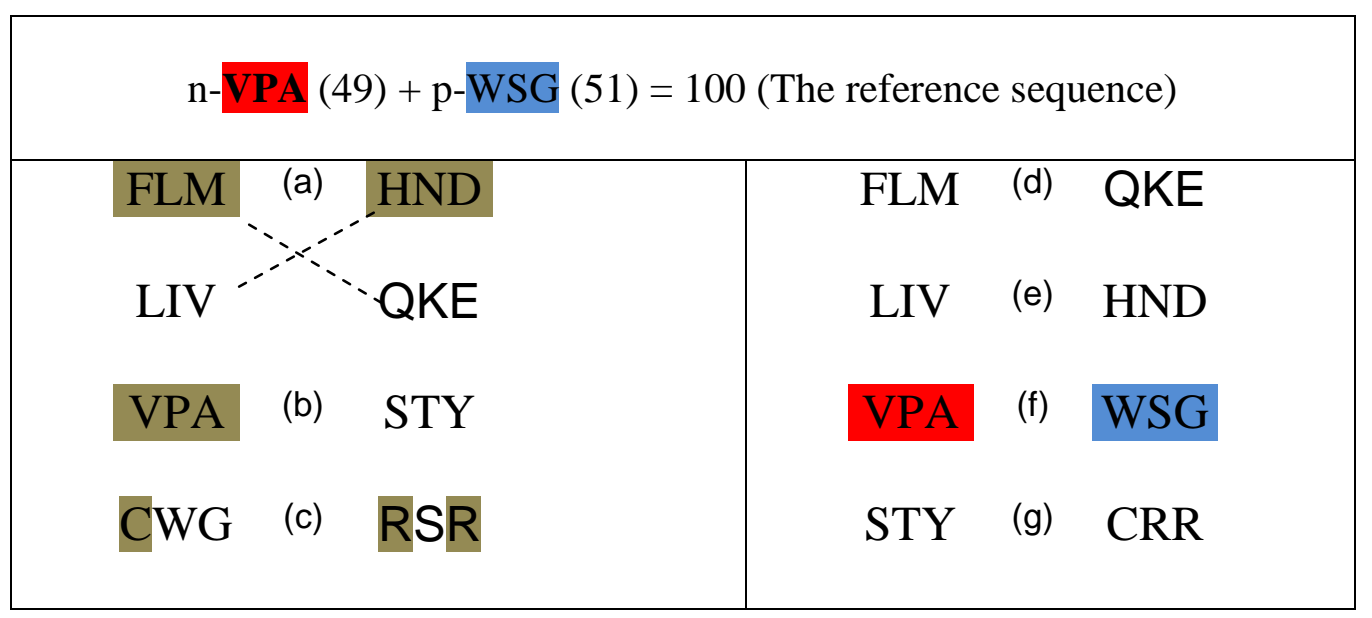

Survey K.1. This survey is the key for the reading and understanding of amino acid arrangement in Tables K.2 and K.3, in odd/even positions, respectively. Also, after our hypothesis (and prediction) - the key of positioning and hierarchy of AAs within proteins. By this the key of the key is the reference sequence (above) with the unity of arithmetical and physico-chemical balance: $50 \pm 1$ of atoms in nonpolar and polar sub-sequence, respectively; balance-nuancing in polarity/nonpolarity: Valine \& Alanine as nonpolar; Proline as nonpolar in polar requirement (Woese, et al., 1966; Konopel'chenko and Rumer, 1975) and polar in hydropathy index (Kyte and Doolittle, 1982) and cloister energy (Swanson, 1984); Serine as polar; Tryptophan and Glycine as polar in hydropathy index, and nonpolar in cloister energy. Except this way, the balance-nuancing is evident through realization of three logics: (a) nonpolar AAs on the left and polar on the right; (b) AAs in odd positions are nonpolar and AAs in even positions are polar; (c) outer AAs, CWG, are nonpolar (in cloister energy) and inner, RS, polar. The arrangement on the right, the sequences d-g, in correspondence with their arithmetical solutions in Table K.3, shows further nuancing and unity of arithmetical balances and polarity / nonpolarity.

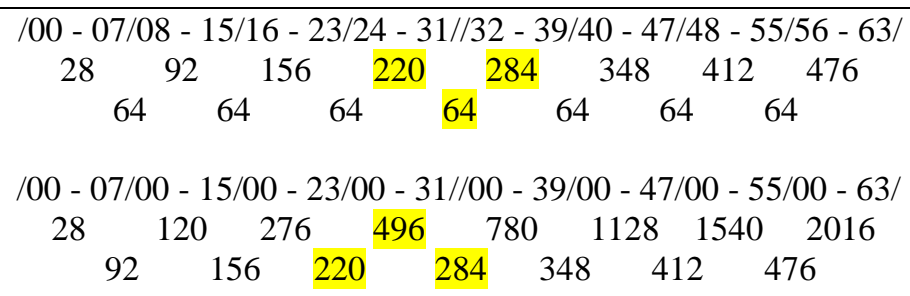

why the genetic code must be six-bit code, no matter if it is the manifestation in the form of the Gray Code model (Swanson, 1984, p 188), or it is in the form of the Binary tree (Rakočević, 1994, p. 38). There must be 8 codon, i.e. amino acid classes. The structure of the sequence $0-63$ is strictly determined by third perfect number (496) and the sum consisted of the first pair of the friendly numbers (220+284)" (Rakočević, 1997b, p. 60). [Cf. with the same pair of friendly numbers, in form 110-284, in Table K.3, below.] 


\section{REFERENCES}

Castro-Chavez, F. (2010) The rules of variation: Amino acid exchange according to the rotating circular genetic code. J. Theor. Biol. 264, 711-721.

Castro-Chavez, F. (2011) The Quantum Workings of the Rotating 64-Grid Genetic Code, Neuroquantology, 9(4), 728-746.

Collins, F.S., 2006. The Language of God, Free Press, Bethesda, Maryland, USA.

Damjanović, M. Z. (1998) Logic core of genetic code, Proceedings of the Section of Natural Sciences on Montenegrin Academy of Sciences and Arts (CANU), 12, 5-8.

Damjanović, M. Z., Rakočević, M. M. (2005) Genetic code: an alternative model of translation, Annals of New York Academy of Sciences, 1048, 517-523.

Damjanović, Z. M., Rakočević, M. M. (2006) Genetic Code: A new understanding of codon amino acid assignment, arXiv:qbio/0611033v1 [q-bio.OT].Darwin, Ch. (1996) On the Origin of Species, Oxford UP.

Darwin, Ch. (1859) On the Origin of Species, John Murray, London.

Darwin, Ch. (1876) The Origin of Species, John Murray, 6th ed., with additions and corrections, London.

Dlyasin, G.G. (1998) Azbuka Germesa Trismegista, ili molekulyarnaya tainopis' myshleniya, Belye Al'vy, Moskva.

Dlyasin, G. G. (2011) Azbuka Germesa Trismegista, ili molekulyarnaya tainopis' myshleniya, Amrita-Rus', Moskva.

Dragovich, B. (2009) Genetic Code and Number Theory, in "Modern Topics in Science", a book of invited papers (Eds. R. Constantinescu, G. Djordjevic, Lj. Nesic), Niš, Serbia; arXiv:0911.4014v1 [q-bio.OT].

Dragovich, B. (2011) p-Adic structure of the genetic code, NeuroQuantology 9(4), 716-727; arXiv:1202.2353v1 [q-bio.OT].

Fleming, Leonore (2013) The Notion of Limited Perfect Adaptedness in Darwin's Principle of Divergence, Perspectives on Science, Spring 2013, Vol. 21, No. 1, Pages 1-22.

Gabora, L. (2013) An evolutionary framework for culture: Selectionism versus communal exchange. Physics of Life Reviews, 10(2), 117-145. 
Jokić, A., Rakočević, M. M. (1996) Four stereochemical types of protein amino acids: synchronic determination with chemical characteristics, atom and nucleon number. J. Theor. Biol. 183, 345 - 349 .

Kauffman, S. (2014) A Holistic, Non-algorithmic View of Cultural Evolution: Commentary on Review Article by Prof. Liane Gabora, arXiv:1410.1836v1 [q-bio.NC].

Kedrov, B.M. (1977) Prognozy D.I. Mendeleyeva v atomistike, Atomizdat, Moskva.

Kyte, J. and Doolittle, R.F. (1982) A simple method for displaying the hydropathic character of a protein, J. Mol. Biol. 157, 105-132.

Konopel'chenko, B. G; Rumer, Yu. B. (1975) Klassifikaciya kodonov v geneticheskom kode. Dokl. Akad. Nauk. SSSR 223, 471-474.

Marcus, S. (1989). Symmetry in the Simplest Case: the Real Line. Computers Math. Applic. 17, 103-115.

Mišić, N. Ž. (2011) Nested numeric/geometric/arithmetic properties of shCherbak's prime quantum 037 as a base of (biological) coding/computing. Neuroquantology, 9, 702-715.

Negadi, T. (2009) The genetic code degeneracy and the amino acids chemical composition are connected, Neuroquantology, Vol. 7, 1, 181-187; arXiv:0903.4131v1 [q-bio.OT].

Negadi, T. (2014) The genetic code invariance: when Euler and Fibonacci meet, Symmetry: Culture and Science, Vol.25, No.3, 145-288, 2014; arXiv:1406.6092v1 [q-bio.OT].

Pullen, S., 2005. Intelligent design or evolution? Free Press, Raleigh, N. Carolina, USA.

Rakočević, M. M. (1991) The coherence of the chemical and genetic code, Proceedings of Faculty of science (before: Faculty of philosophy), Chemistry Section, 2, 1-29, Niš.

Rakočević, M. M. (1994). Logic of the Genetic Code, Naučna knjiga, Belgrade.

Rakočević, M.M., (1997a) Two classes of the aminoacyl-tRNA synthetases in correspondence with the codon path cube. Bull. Math. Biol. 59, 645-648.

Rakočević, M. M., (1997b) Genetic code as a unique system. SKC, Niš (www.rakocevcode.rs)

Rakočević, M.M. (1998a) The genetic code as a golden mean determined system, Biosystems 46, 283-291.

Rakočević, M. M. (1998b) Whole-number relations between protein amino acids and their biosynthetic precursors, J. Theor. Biol. 191, $463-465$.

Rakočević, M. M. (2004) A harmonic structure of the genetic code, J. Theor. Biol. 229, 463-465. 
Rakočević, M. M. (2008) Genetic Code: Four-Codon and Non-Four-Codon Degeneracy, arXiv:0802.1056v1 [q-bio.BM]

Rakočević, M. M. (2009) Genetic Code: The unity of the stereochemical determinism and pure chance, arXiv:0904.1161v1 [q-bio.BM].

Rakočević, M. M. (2011a) Genetic Code: Four Diversity Types of Protein Amino Acids, arXiv:1107.1998v2 [q-bio.OT]

Rakočević, M.M. (2011b) Genetic code as a coherent system, Neuroquantology 9, 821-841. (www.rakocevcode.rs)

Rakočević, M. M. (2013) Harmonic mean as a determinant of the genetic code, arXiv:1305.5103v4 [q-bio.OT].

Shcherbak, V. I. (1993) Twenty canonical amino acids of the genetic code: the arithmetical regularities, part I, J Theor. Biol., 162, 399-401.

Shcherbak, V.I. (1994) Sixty-four triplets and 20 canonical amino acids of the genetic code: the arithmetical regularities. Part II. J. Theor. Biol. 166, 475-477.

Shcherbak, V. I. (2008) The arithmetical origin of the genetic code, in: The Codes of Life, Springer.

Swanson, R. (1984) A unifying concept for the amino acid code. Bull. Math. Biol. 46, 187-207.

Timiryazev, K. (1942) Istoricheskij metod v biologii, Akademiya nauk SSSR, Moskva.

Verkhovod, A. B. (1994) Alphanumerical divisions of the universal genetic code: new divisions reveal new balances, J. Theor. Biol., 170, 327-330.

Woese, C.R. et al. (1966) On the fundamental nature and evolution of the Genetic Code. In: Cold Spring Harbor Symp. Quant. Biol. 31, 723-736. 